\title{
MASTER
}

\section{Summary of EBT-I Experimental Results}
F. W. Baity, Jr.
M. C. Becker
K. H. Carpenter
J. A. Cobble
H. O. Eason
M. Fujiwara
J. C. Glowienka
G. R. Haste
M. E. Hesse
S. Hiroe

R. A. Dandl

H. Ikegami

H. W. Moos

J. M. Tyson

E. S. Warden

N. H. Lazar

R. L. Livesey

M. W. McGuffin

D. H. McNeill

B. H. Quon

J. W. Reynolds
F. M. Bieniosek

F. J. Bresnock

P. L. Colestock

K. A. Connor

R. L. Hickok

S. S. Kuo

W. J. Schill

T. Uckan

T. L. White

R. E. Wintenberg

\section{OAK RIDGE NATIONAL LABORATORY} OPERATED BY UNION CARBIDE CORPORATION · FOR THE DEPARTMENT OF ENERGY 


\section{DISCLAIMER}

This report was prepared as an account of work sponsored by an agency of the United States Government. Neither the United States Government nor any agency Thereof, nor any of their employees, makes any warranty, express or implied, or assumes any legal liability or responsibility for the accuracy, completeness, or usefulness of any information, apparatus, product, or process disclosed, or represents that its use would not infringe privately owned rights. Reference herein to any specific commercial product, process, or service by trade name, trademark, manufacturer, or otherwise does not necessarily constitute or imply its endorsement, recommendation, or favoring by the United States Government or any agency thereof. The views and opinions of authors expressed herein do not necessarily state or reflect those of the United States Government or any agency thereof. 


\section{DISCLAIMER}

Portions of this document may be illegible in electronic image products. Images are produced from the best available original document. 
Printed in the United States of America. Available from National Technical Information Service

U.S. Department of Commerce

5285 Port Royal Road, Springfield, Virginia 22161

Price: Printed Copy $\$ 6.00$; Microfiche $\$ 3.00$

This report was prepared as an account of work sponsored by an agency of the United States Government. Neither the United States Government nor any agency thereof, nor any of their employees, contractors, subcontractors, or their employees, makes any warranty, express or implied, nor assumes any legal liability or responsibility for any third party's use or the results of such use of any information, apparatus, product or process disclosed in this report, nor represents that its use by such third party would not infringe privately owned rights. 
Contract No. W-7405-eng-26

FUSION ENERGY DIVISION

SUMMARY OF EBT-I EXPERIMENTAL RESULTS

EBT Experimental Group

Date Published: October 1978

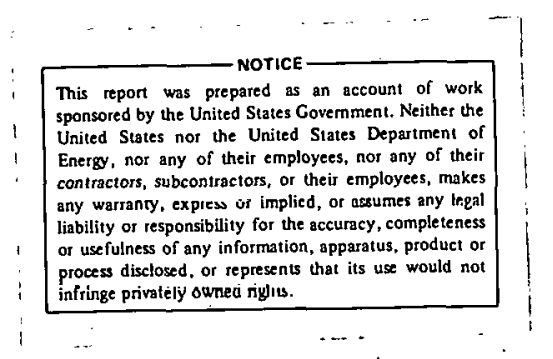

NOTICE This document contains information of a preliminary nature. It is subject to revision or correction and therefore does not represent a final report.

\author{
Prepared by the \\ OAK RIDGE NATIONAL LABORATORY \\ Oak Ridge; 'l'ennessee 37830 \\ operated by \\ UNION CARBIDE CORPORATION \\ for the \\ DEPARTMENT OF ENERGY
}


THIS PAGE

WAS INTENTIONALLY

LEFT BLANK 
EBT Experimental Group*

R. A. Dandl, F. W. Baity, Jr., M. C. Becker, ${ }^{1}$ K. H. Carpenter, ${ }^{2}$

J. A. Cobble, H. O. Eason, M. Fujiwara, ${ }^{3}$ J. C. Glowienka, G. R. Haste, M. E. Hesse, ${ }^{4} \mathrm{~S}$. Hiroe, ${ }^{3} \mathrm{H}$. Ikegami, ${ }^{3}$ JHU Vacuum UV Group, ${ }^{5}$ N. H. Lazar, R. L. Livesey,

M. W. McGuffin, D. H. McNeill, 6 B. H. Quon, J. W. Reynolds, 1 RPI Heavy Ion Beam Group, 7 W. J. Schill, 8 T. Uckan,

T. L. White, R. E. Wintenberg

${ }^{1}$ Instrumentation and Controls Division

'Computer Sciences Divisinn

${ }^{3}$ Institute of Plasma Physics, Nagoya, Japan

${ }^{4}$ On lcave from Centre d'Etudes Nucleaires de Grenoble, Grenoble, France

JThe Johns Hopkins University: H. W. Moos, J. M. Tyson, and E. S. Warden

${ }^{6}$ Now with the TES section

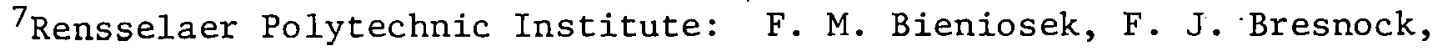
P. L. Colestock, K. A. Connor, R. L. Hickok, S. S. Kuo

${ }^{8}$ Now with ARAMCO, Saudi Mrabia 


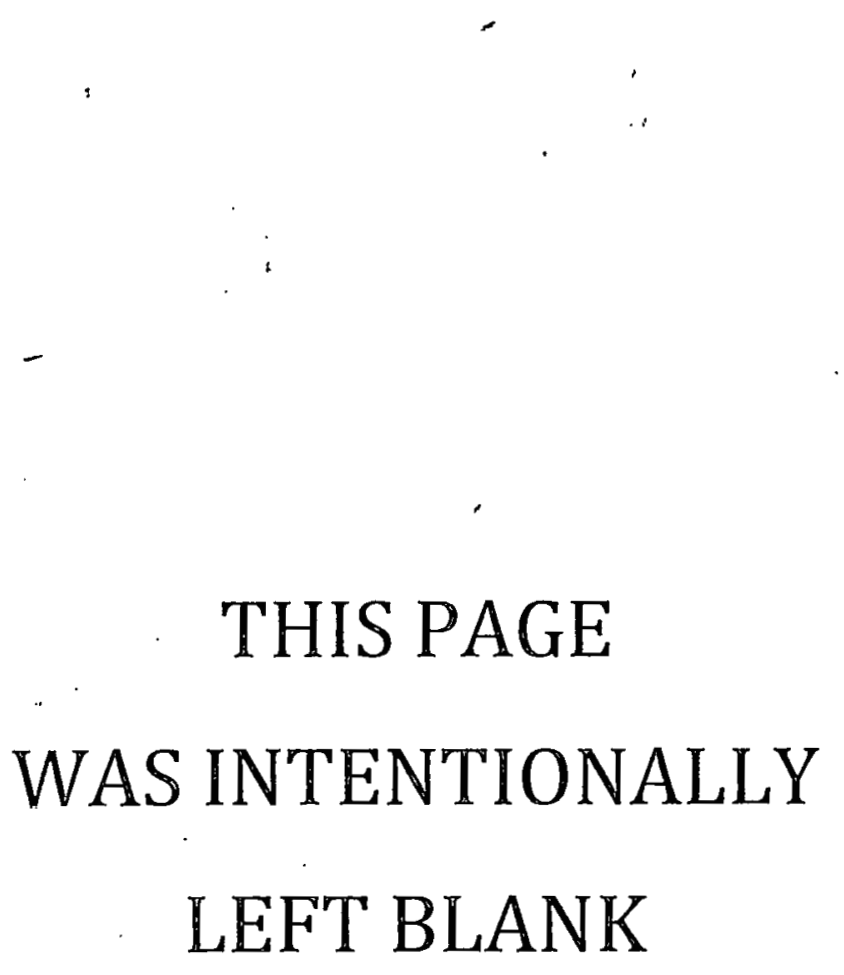


CONTENTS

ABSTRACT . . . . . . . . . . . . . . . . . . . . . vii

SUMMARY OF EBT-I EXPERIMENTAL RESULTS . . . . . . . . . . . . . . 1

1. PHYSICAL DESCRIPTION AND OPERATION . . . . . . . . . . 3

2. EARLY EXPERIMENTS IN EBT-I . . . . . . . . . . . . . . . 9 9

2.1 DEMONSTRATION OF VALIDITY OF EBT CONCEPT . . . . . . . . . 9

2.2 MEASUREMENT OF THE LOCATION OF THE TOROIDAL PLASMA . . . . 12

3. STABILITY OF THE ELECTRON ANNULUS . . . . . . . . . . . . . 17

4. MEASUREMENT OF PlaSMA PARAMETERS IN T-MODE . . . . . . . . . . . 19

4.1 ELECTRON DENSITY . . . . . . . . . . . . . . . . . . 19

4.2 ELECTRON TEMPERATURE . . . . . . . . . . . . . . . 19

4.3 ION TEMPERATURE . . . . . . . . . . . . . . . . . . 21

4.4 SPACE POTENTIAL MEASUREMENT . . . . . . . . . . . . . . 29

4.5 IMPURITIES . . . . . . . . . . . . . . . . 33

4.6 NEUTRAL HYDROGEN . . . . . . . . . . . . . . . . . 40

4.7 TOROIDAL CURRENTS AND FIELD ERROR EFFECTS ON EBT . . . . . 45

5. DISCUSSION OF MEASUREMENTS . . . . . . . . . . . . . . . 55

5.1 ESTIMATE OF TOROIDAL PLASMA CONFINEMENT TIME . . . . . . 55

5.2 PARAMETRIC STUDIES . . . . . . . . . . . . . 59 REFERENCES ............................ . . . 71 
THIS PAGE

WAS INTENTIONALLY

LEFT BLANK 


\begin{abstract}
Experiments in the ELMO Bumpy Torus (EBT-I) are described which demonstrate that plasma currents produced by microwave heated, hotelectron annuli can produce macroscopically stable plasma confinement in a steady-state bumpy torus. The diagnostic developments which provide the array of tools required to document the plasma properties are described. The toroidal plasma parameters were measured over a modest range of operating conditions and are categorized by the theoretically relevant parameter $\nu / \Omega$, where $\nu$ is the electron collision frequency and $\Omega$ is the average electron precession frequency about the toroidal axis. The relevance of the theoretical transport model is discussed in a comparison with the data.
\end{abstract}


From the reactor design point of view, the bumpy torus confinement geometry has many attractive features: symmetrical magnet coils, convenient access and shielding because of large aspect ratio, and the possibility of steady-state operation. However, even the most superficial examination of the unfavorable magnetic field curvature leads one to suspect that the plasma would be MHD unstable. In 1971 we formally proposed a specific bumpy torus, ${ }^{1}$ the ELMO Bumpy Torus (EBT-I), designed to circumvent the MHD stability problem by using the diamagnetism of energetic electron annuli produced by electron cyclotron heating to modify the unfavorable magnetic curvature.

Starting in 1972 , EBT-I was constructed to examine the following basic questions:

(a) Can stable, diamagnetic electron annuli be efficiently formed by electron cyclotron heating in bumpy torus geometry?

(b) Do the rings form so as to permit a stable plasma equilibrium?

(c) If and when the annuli form, is the toroidal plasma confined stably by the resultant modification of the magnetic curvature?

Experiments were begun in September 1973, and almost immediately affirmative responses were found to each of the three fundamental questions. The evidence for these conclusions is presented in Sect. 2 . In Sect. 3, a short summary of the observations related to the stability of the annulus in EBT-I is included.

When these proof-of-principle experiments were affirmatively completed, a series of studies using the available heating power ( $30 \mathrm{~kW}$ at $18 \mathrm{GHz}$ ) was initiated to document the plasma parameters in the stable, toroidally confined regime. It was soon determined that an extensive diagnostic development program was required because the plasma parameters fell in a range which stressed the technological limits of the conventional measurement techniques. At the same time, simple estimates of confinement properties indicated that with higher applied power a modest improvement in plasma temperatures could be expected, thus alleviating 
to some extent the diagnostic difficulty; in addition, initial scaling of these properties with power would become possible.

As a consequence, experiments aimed at detailed studies of plasma parameters were initiated with the added flexibility resulting from a doubling of the maximum available $18-\mathrm{GHz}$ microwave power. The further addition of a $30-\mathrm{kW} \mathrm{cw}, 10.6-\mathrm{GHz}$ microwave power source permitted independent control over the diamagnetism of the annular electron component as well as providing lower off-resonant heating of the toroidal plasma. With this heating configuration, and after further technological upgrading of the entire diagnostic array, the plasma parameters in the stable toroidal plasma were documented (see Sect. 4). The relatively good confinement of these higher density $\left(\mathrm{n}_{\mathrm{e}} \geq 10^{12} \mathrm{~cm}^{-3}\right.$ ), higher temperature $\left(\mathrm{T}_{\mathrm{e}} \sim 300 \mathrm{eV}, \mathrm{T}_{i} \sim 100 \mathrm{eV}\right)$, toroidal plasmas inspired very productive theoretical modeling efforts. The resulting plasma models led to predicted plasma properties which looked very attractive when extrapolated to higher magnetic fields and applied power. These properties depend sensitively on magnitude and direction of the ambipolar electric field which is self-consistently generated. Although some efforts at potential measurements were made in the early studies, the plasma models clearly indicated that a wider range of measurements should be undertaken, particularly, with greater accuracy.

The result of these measurements was a modification of the theoretical models and, consequently, a deeper understanding of the fundamental confinement process in EBT-I (and its possible successors).

In the following discussion we describe the results of the EBT-I experiments during its four years of existence. In Sect. 5, the data are compared to the currently best developed theoretical model of the toroidally confined plasma, and the agreements and shortcomings of both the model and experiments are described. Some indication of where extrapolation to higher magnetic fields and power leads is outlined, but the need for a modest scaling experiment is self-evident in order to provide a basis of credibility for greater extrapolations of physical parameters. The experiment EBT-S is designed to provide this credibility. 


\section{PHYSICAL DESCRIPTION AND OPERATION}

The physical dimensions and operational parameters of EBT-I are listed in Table 1. A layout of the facility is shown in Fig. 1, and a photograph of the machine is shown in Fig. 2. The magnetic field was produced by 24 magnetic coils arranged about a torus, each pair producing a 2:1 magnetic mirror ratio on the axis. The aluminum vacuum chamber in each toroidal bump was pumped into a toroidal vacuum manifold connected to four baffled, $25-\mathrm{cm}$ diffusion pumps operated with highquality "Santovac" pump oil. Each magnetic mirror sector was fed with. microwave power at both $10.6 \mathrm{GHz}$ and $18 \mathrm{GHz}$ - from a distribution system comprised of four 18-GHz klystron amplifiers and three 10.6-GHz klystron amplifiers.

The power at both frequencies was controlled by adjusting the output drive power of the oscillator to the high power output klystron amplifiers. Additional control of the $18-\mathrm{GHz}$ power into each toroidal sector was provided by a ferrite attenuator device which effectively shunted any fraction of the applied sector power into a dummy load. These independent controls were designed into the system in case difficulties arose due to imbalance of power around the torus. Although this worry proved to be unfounded, the remotely controlled cavity power level controls were of great diagnostic convenience. The entire facility was contained in a 15-cm thick, lead-walled room for personnel protection against the hard $x$-rays resulting from energetic annulus electrons striking cavity walls.

An equatorial plane view of one toroidal section is shown in Fig. 3. Both magnetic flux lines and surfaces of constant $|B|$ are indicated. To operate EBT, the magnitude of the magnetic field was adjusted so that bulk heating would occur on cyclotron resonance magnetic surfaces approximately midway between the minimum field in the midplane and the maximum field, as illustrated in Fig. 3. Because of the usual negative radial magnetic gradient of simple mirror geometry, second harmonic gyrofrequency resonant surfaces exist at the half field value which is illustrated in Fig. 3 at a radius of $15 \mathrm{~cm}$. Electrons passing 
Table 1. Nominal EBT parameters

\section{Present EBT}

\begin{tabular}{ll} 
Magnetic field & $6.5 \mathrm{kG}$ midplane - $13 \mathrm{kG}$ mirrur \\
Magnetic field power & $10 \mathrm{MW}$ \\
Torus volume & $\cong 1,351$ liters \\
Major radius & $150 \mathrm{~cm}$ \\
Aspect ratio & $=10: 1$ \\
cw microwave power & \\
ECH microwave source & $60 \mathrm{~kW} \mathrm{cw}-18 \mathrm{GHz}$ \\
LORH microwave source & $30 \mathrm{~kW} \mathrm{cw}-10.6 \mathrm{GHz}$ \\
\hline
\end{tabular}




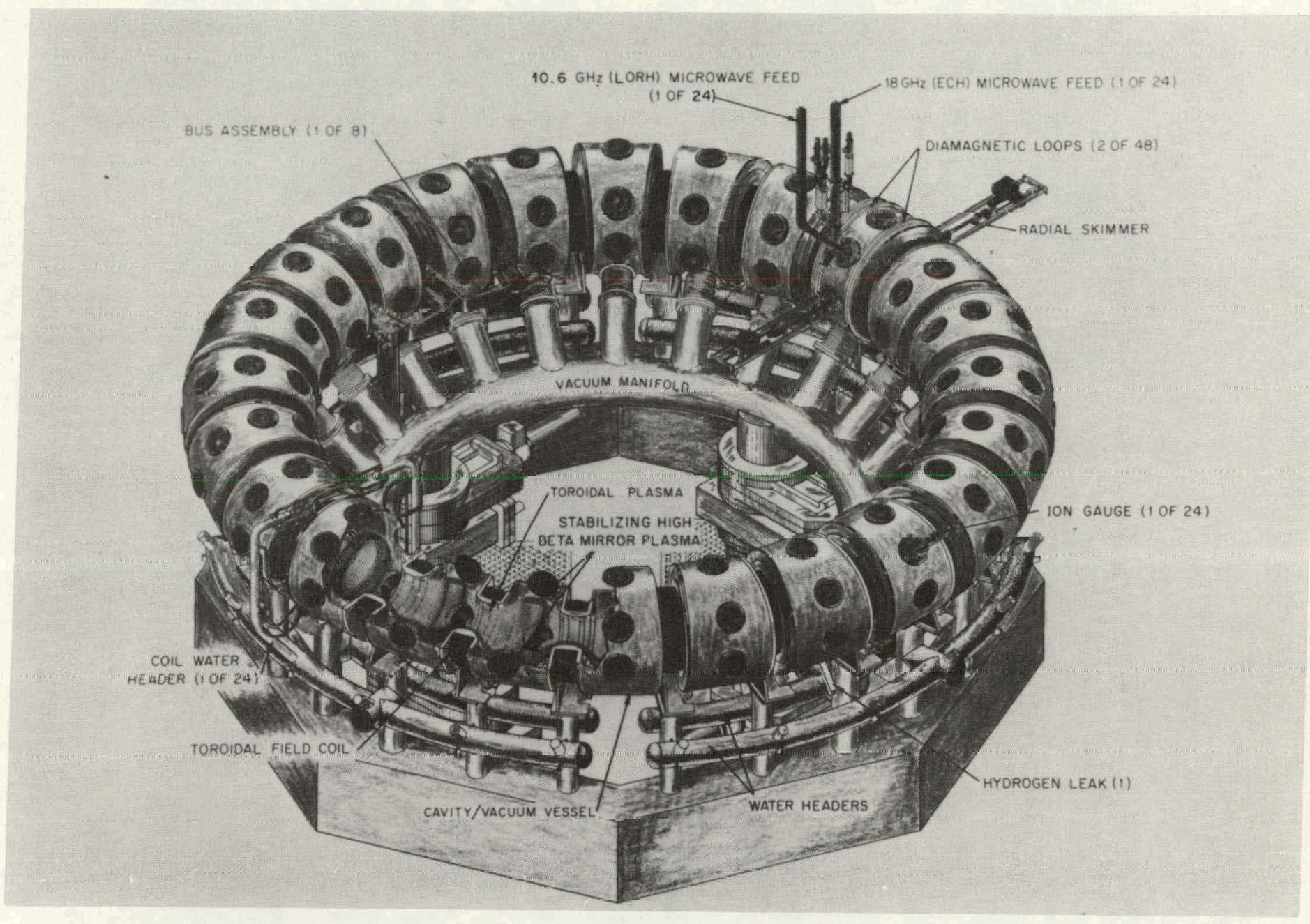

Fig. 1. Schematic layout of EBT-I. 


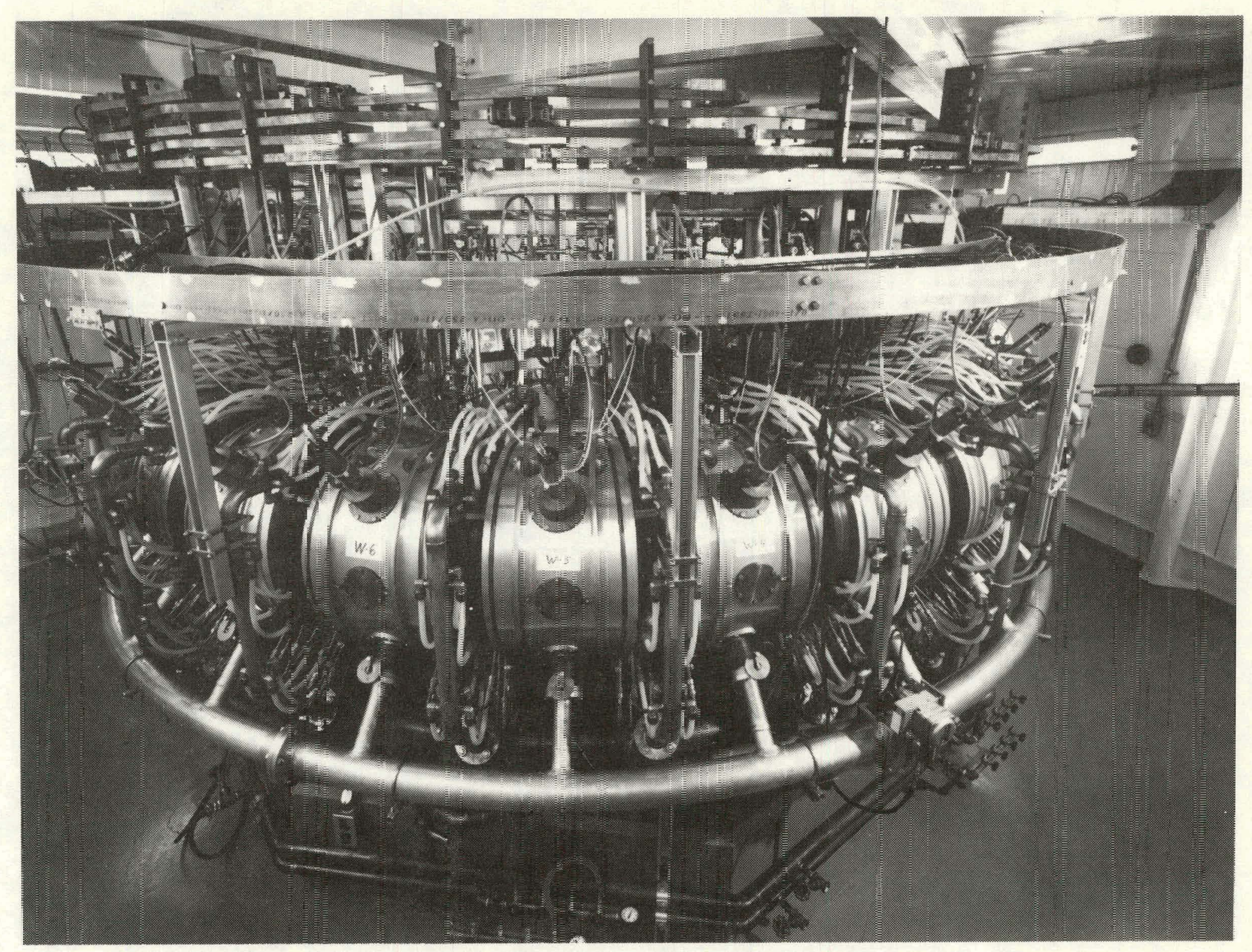

Fiz. 2. Photograph of EET-I. 


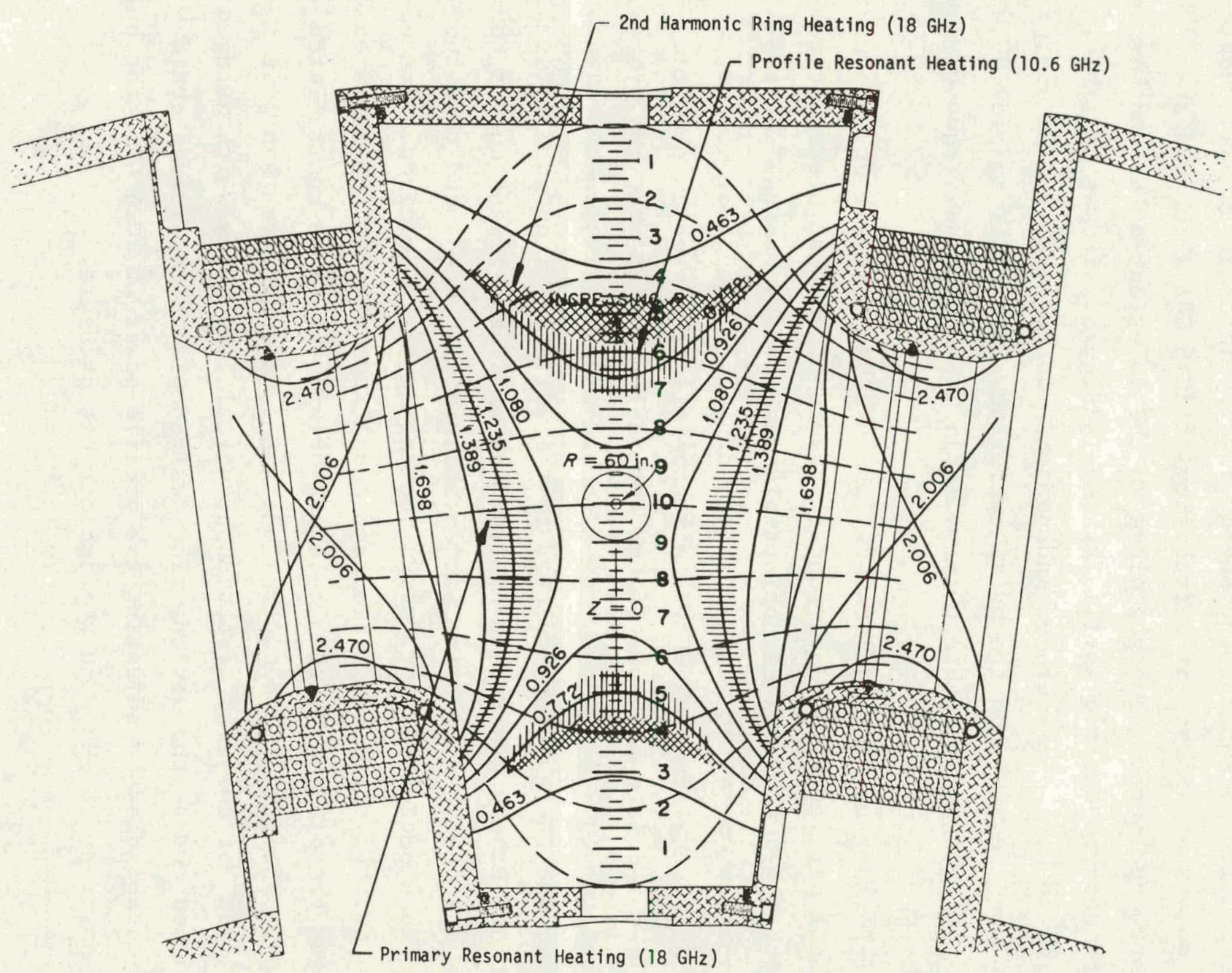

Fig. 3. Section through EBT-I in the horizontal midplane. Solid lines indicate surfaces of constant $|B|$, and dotted lines indicate flux lines. Shaded areas are regions of cyclotron resonance for electrons when $\mathrm{B}_{\mathrm{O}}=4.55 \mathrm{kG}$. 
through these surfaces are in resonance at the second harmonic of the electron cyclotron frequency and are observed to be effectively heated to high energies - thus producing annuli of precessing encrgetic plasma at these radii. To control the energy density in these annuli, supplementary power was applied at a frequency of $10.6 \mathrm{GHz}$ which was at fundamental cyclotron resonance close to the locations of second harmonic resonance described above as illustrated in Fig. 3. It was possible, therefore, to have a measure of independent control over stored energy of the annulus (i.e., $\beta$ ) and the bulk heating power in order to provide a range of operating parameters not available from a single frequency source alone.

EBT-I was generally operated $\mathrm{cw}$, and feedback control of the critical operational parameters was provided to assure a constant steady state. To operate the device the magnetic field was adjusted to the desired level, and the operating gas, usually hydrogen (although deuterium, helium, nitrogen, argon, and even air have all been used as operating gases), was fed into only one of the toroidal bumps so that the pressure was raised to $\sim 10^{-5}$ torr. As the microwave power was raised, a plasma was formed and the gas recirculated to the walls until an equilibrium was reached between gas returning from the walls and losses out of the plasma. When this equilibrium was established, it was found that the ion gages in each sector read the same, indicating that the circulation of plasma about the torus had introduced equal losses to the walls in each sector and the plasma was then in equilibrium with these recirculated particles. The fast servo-controlled, gas-feed input was then just a "makeup" gas for the small fraction of circulating atoms which managed to be pumped out of the device. The desired plasma conditions could then be established by adjusting the profile power at $10.6 \mathrm{GHz}$, the bulk heating power at $18 \mathrm{GHz}$, and the ambient gas pressure. 


\section{EARLY EXPERIMENTS IN EBT-I}

\subsection{DEMONSTRATION OF VALIDITY OF EBT CONCEPT}

Previous to the ELMO Bumpy Torus (EBT-I), confinement of plasma in a spatially, periodically-modulated toroidal magnetic field (bumpy torus) had received very little experimental attention because of anticipated MHD instability as a consequence of the unfavorable magnetic field gradients. In EBT-I it was proposed to stabilize the toroidal plasma by generating poloidal plasma currents in steady state which would cancel the negative field gradients in each "bump" of the torus. These currents were expected to arise from the diamagnetism resulting from annuli of energetic electrons which are a consequence of electron cyclotron heating in this geometry, as was observed in the early ECH mirror machine experiments at ORNL.

Construction of the EBT facility was approved in 1972 and completed in September 1973. Initial experiments were begun with microwave power fed into only four toroidal bumps to examine the conditions required to establish stable, diamagnetic annuli in this novel, toroidal configuration. On the very first day of experiments (September 13, 1973) finite stored energy in the four cavities was observed and found to decay stably when the power was interrupted (Fig. 4).

There followed a period of intense activity in which the next critical question was addressed, namely, does the diamagnetism of the hot electron annulus modify the magnetic curvature sufficiently to stabilize the toroidal plasma?

An affirmative response to this question was determined by examining the level of density fluctuations seen in a microwave interferometer as a. function of stored energy $\left(W_{1}\right)$ contained in the electron annulus (Fig. 5). At high neutral pressure and modest microwave heating power, $\mathrm{W}_{\perp} \approx 0$, but a significant average plasma density $\left(n_{e} l\right)$ was measured with the interferometer. The fluctuation level under these conditions was high $[\Delta(n l) /(n l) \approx 1]$ in the video-frequency band of the interferometer $(10 \mathrm{~Hz} \rightarrow 30 \mathrm{kHz})$. This regime of operation was designated the C-mode (for cold plasma operation). As the neutral pressure was lowered, the stored energy in each sector increased (the energy in one particular 
Su power

THERMONUCLEAR DIVISION

DATA SHEET

Sept $13 / 73$

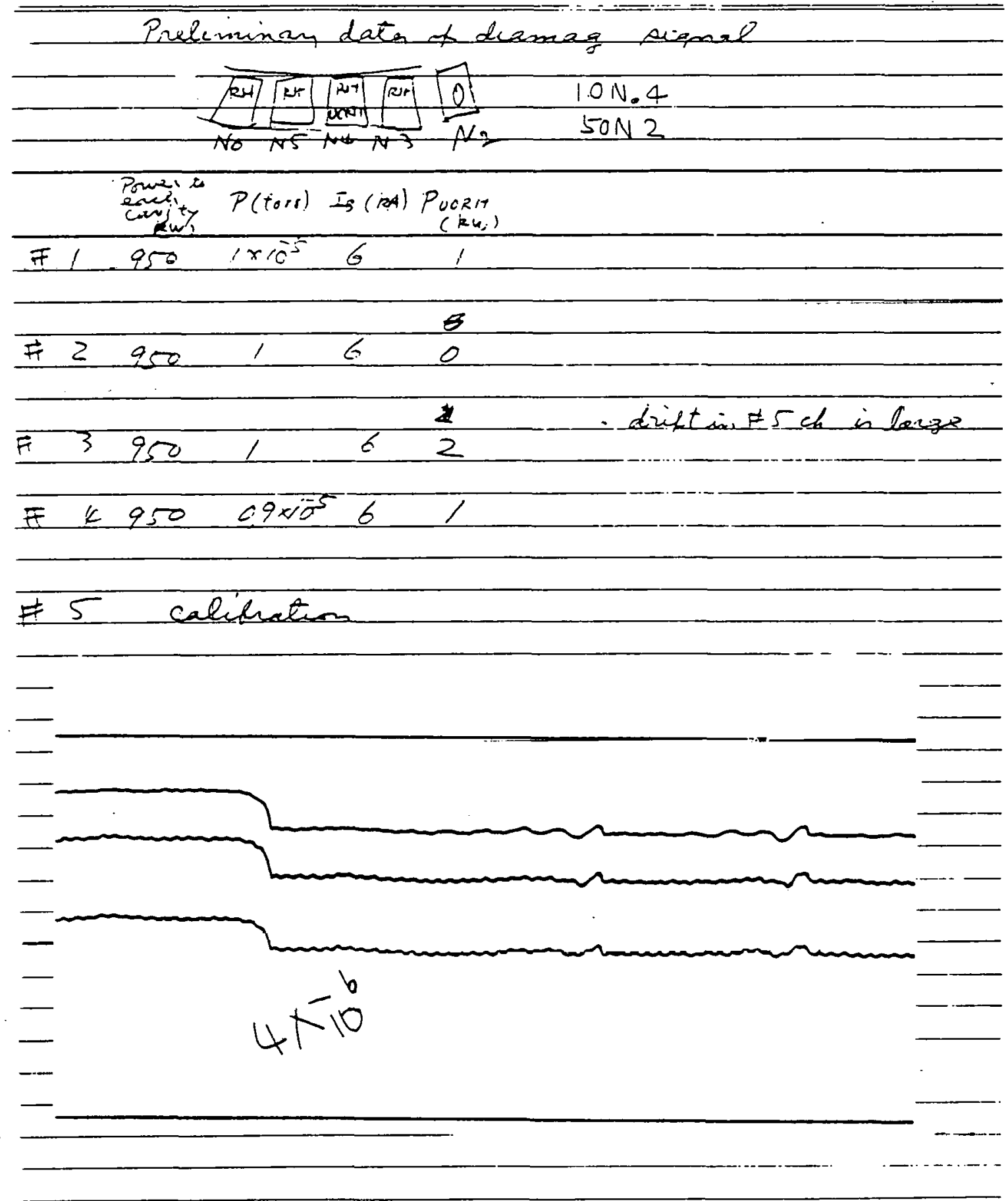

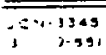

Fig. 4. Data sheet of September 13, 1973 showing $950 \mathrm{~W}$ of 18-GHz power delivered to each of four toroidal sectors. Shown at the bottom are tracings from diamagnetic loops in three of the sections indicating decay of diamagnetism following turnoff of plasma. 
ORNL-DWG $74-11581$

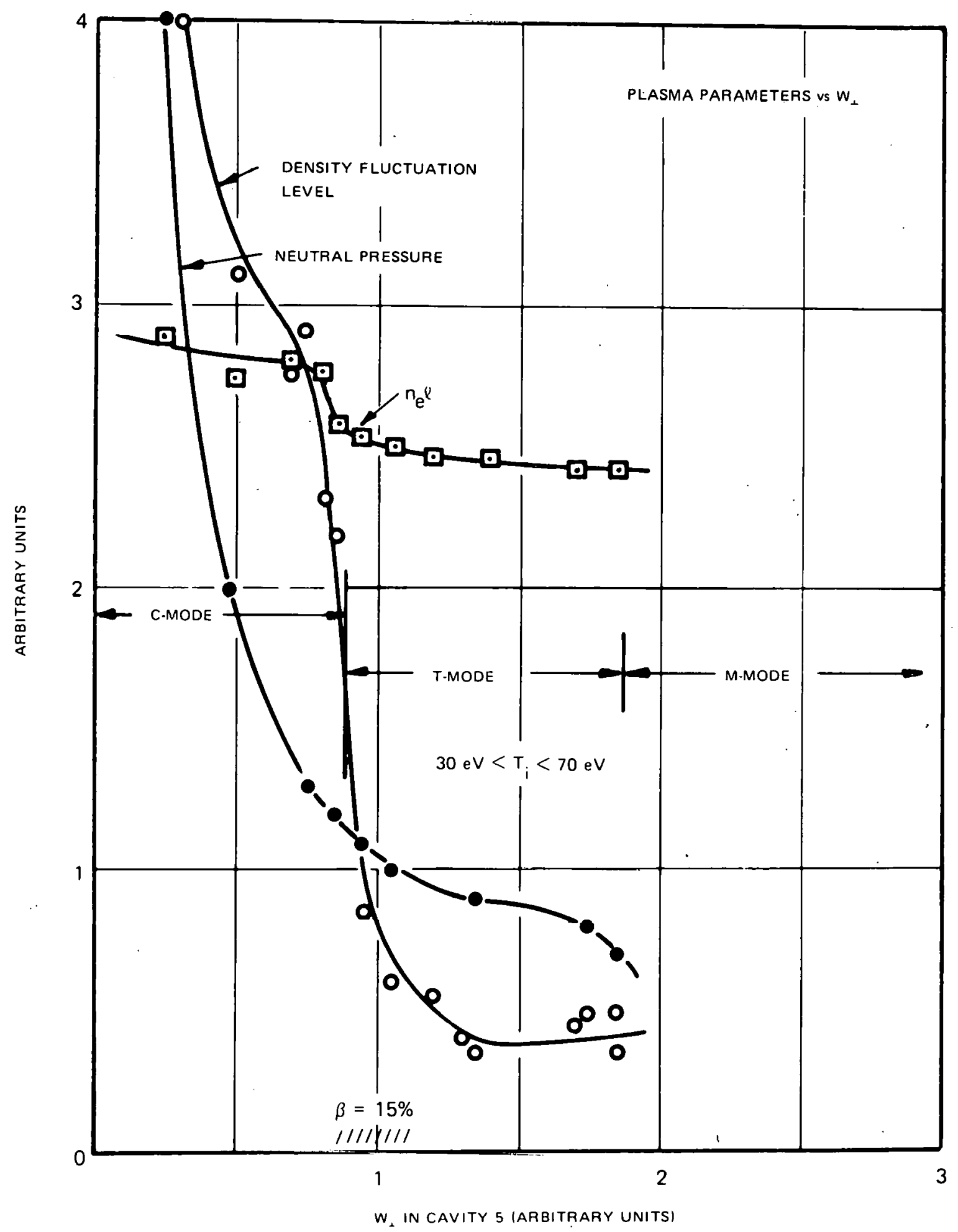

Fig. 5. Plasma parameters measured in EBT-I as a function of observed stored energy $W_{1}:(\bullet)$ neutral pressure, (o) density fluctuation level, (u) $n e^{\ell-1 i n c ~ d e n s i t y . ~}$ 
sector is illustrated in Fig. 5), nl fell slightly as the feed gas was reduced, but the fluctuation level $[\Delta(n l) / n l]$ fell dramatically to almost thermal levels in optimum cases $\left(\Delta n \ell / n \ell \sim 10^{-3}\right)$ when $\beta \geqslant 0.1$, a value consistent with theoretical predictions of the requirements for stability of a toroidally confined Maxwellian plasma. In this regime, designated $T$-mode (for toroidal plasma), the electron and ion temperatures were found to be relatively high. In Fig. 5 this is illustrated by $30<\mathrm{T}_{i}<70 \mathrm{eV}$ determined from the measured energy distribution of charge-exchange neutrals. These facts constituted a demonstration of proof-of-principle of the ELMO Bumpy Torus concept.

\subsection{MEASUREMENT OF THE LOCATION OF THE TOROIDAL PLASMA}

The physical size and location of the plasma was measured using several diagnostics. The location of the diamagnetic annulus was determined by inserting a water-cooled skimmer probe into one of the toroidal sectors both from the outside and inside of the torus. The probe contained an ionization chamber which was sensitive to locally generated $x$-rays produced as the probe intercepted the energetic electrons in the annulus. In Fig. 6, the observed current in the ionization shamber is shown as a function of the probe location. Also shown is the observed diamagnetic flux measured in that sector. At $B_{00}=0.5 \mathrm{~T}$ (magnetic field strength at a coil-pair midplane on axis), the presence of energetic electrons was measured in to a radius of $\sim 12 \mathrm{~cm}$ when the probe was inserted from the outside wall, and the diamagnetic flux half-value point was $r \approx 13 \mathrm{~cm}$. The outer point where energetic electrons were intercepted was $\mathrm{r} \approx 20 \mathrm{~cm}$. Detailed analysis of the shape of the pressure profile which produced these data is complicated by changes in equilibria produced by the probe itself, but a simple plot of the range of $\Delta \phi$ as a function of magnet1c f.ield is shown In F1g. 7. Also drawn is a curve of the location of points where the values of magnetic field in the midplane are at the second harmonic of electron cyclotron resonance for the applied 18-GHz power. The experimental points indicate that the hot electrons were found near these locations and the relatively narrow radial extent demonstrates the annular character of this component of the plasma. 


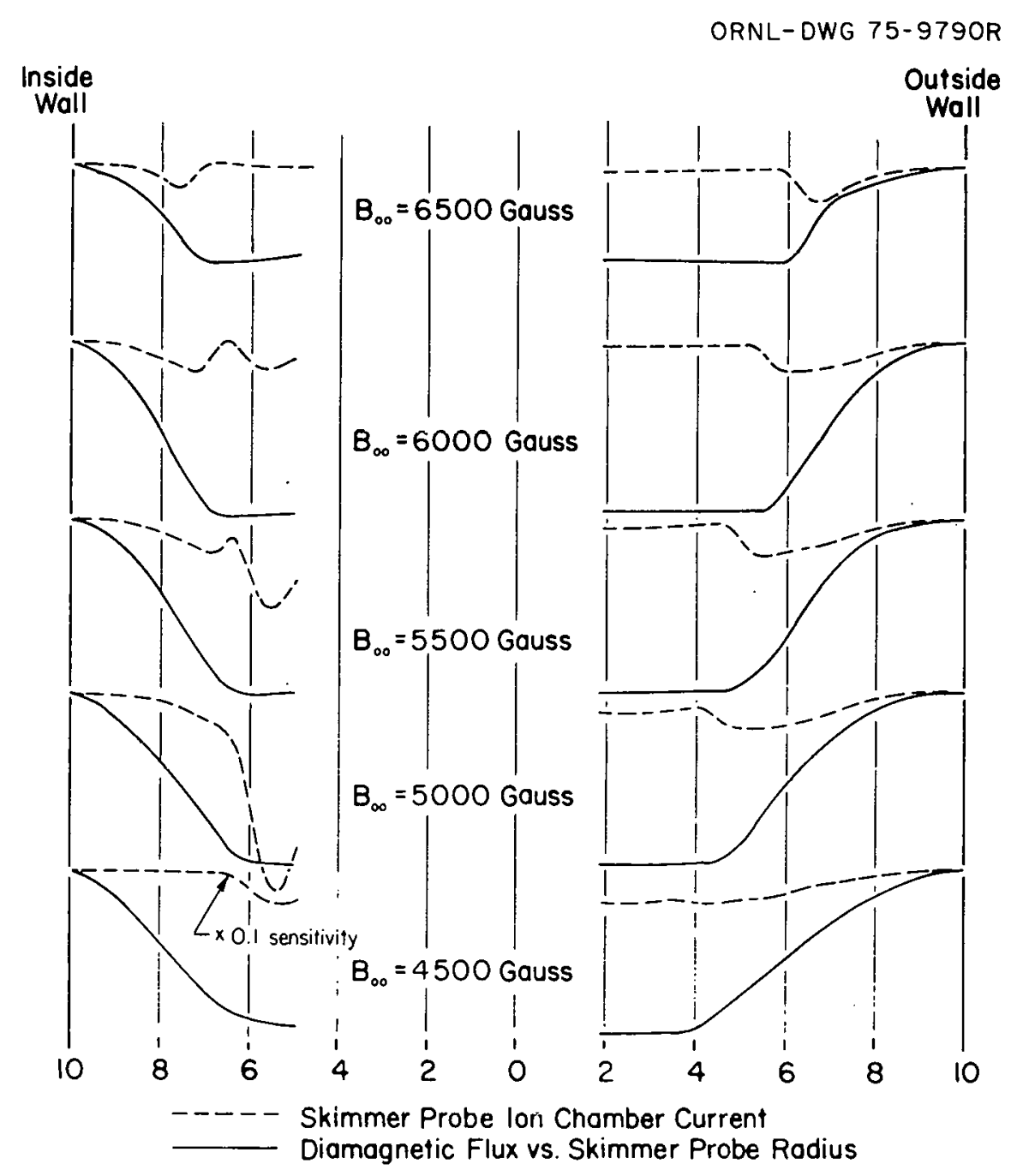

Fig. 6. Measurements of location of annulus with various magnetic field strengths using a skimmer probe containing an ionization chamber to intercept energetic electrons: ( $\rightarrow$ ) observed diamagnetic flux as a function of probe position (---) ion chamber current. 


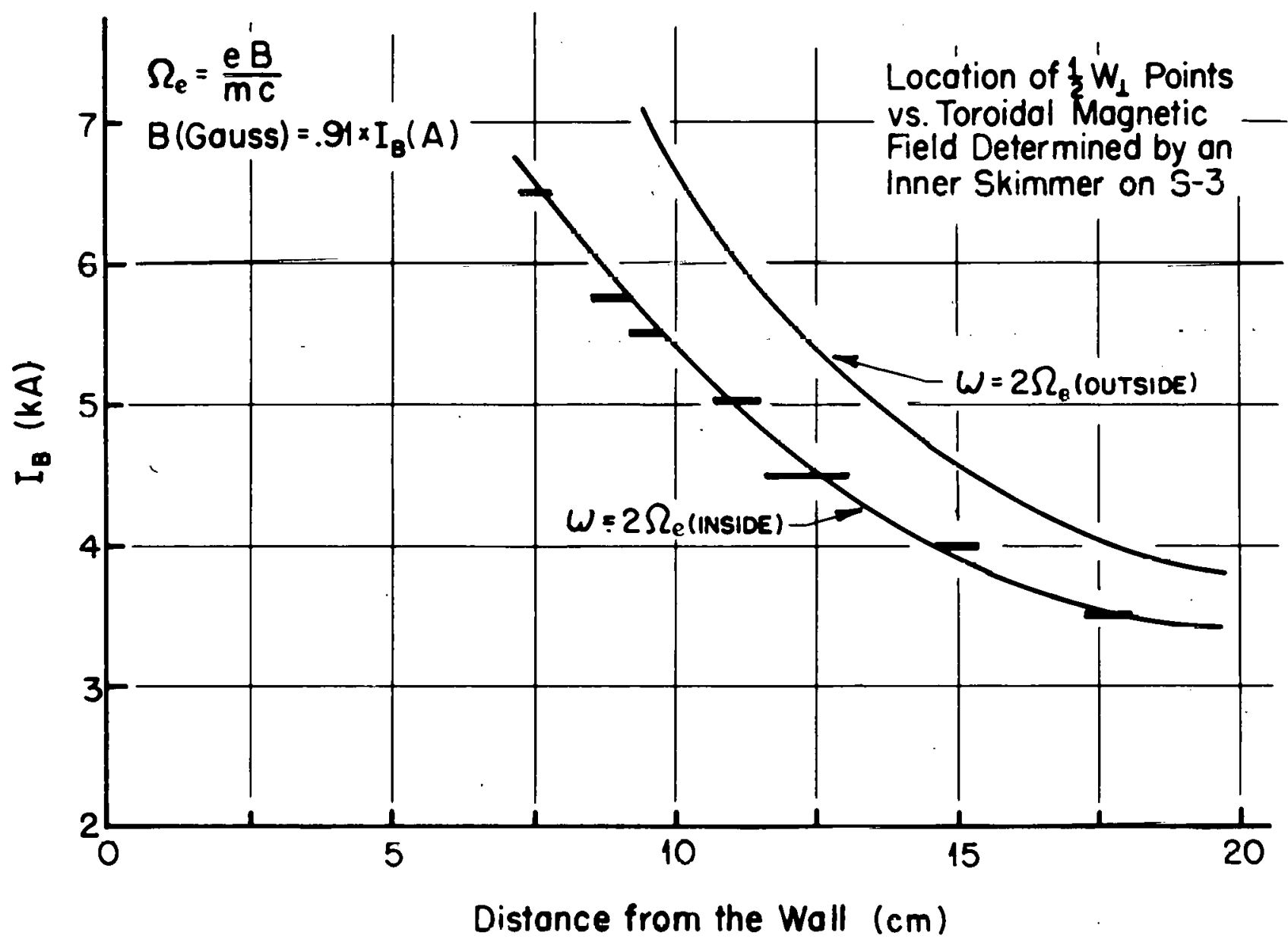

Fig. 1. Location of electron annulus from data in Fig. 6 as a function of magnetic field ( $I_{B}=$ magnet curront). The oolid curves indicate calculated locations for $\omega=2 \Omega$ e for the applied frequency $(\omega=18 \mathrm{CHz})$. 
The location of the toroidally confined plasma was determined from measurements of ion saturation current to a Langmuir probe (in a nonpowered toroidal sector). The current observed as a function of radial position is shown in Fig. 8. For comparison, also shown are the calculated drift surfaces for passing particles (i.e., surfaces of constant $J=\oint \mathrm{p}_{\|} \mathrm{ds}$ with $v=v_{\|}$in the midplane). The probe (although water-cooled) overheated when inserted too close to the axis, so data were only available for $r \geqslant 10 \mathrm{~cm}$ on the outside of the torus. Clearly, the maximum observed saturation ion current lay well within the electron annuli and approximately in the vicinity of closed drift surfaces. Later measurements showed the existence of toroidally directed fast electrons within the region of closed drift surfaces when the magnetic field errors were not cancelled (as in these experiments) and this may account, in part, for the low currents seen at the center of the region with closed drifts. In any case, from these measurements the toroidal plasma was spatially located inside the diamagnetic annuli and was shown to flow stably about the torus. In addition to saturation current, floating potential was determined as a function of position. These early probe floating potential measurements gave first indications of the ambipolar potential behavior as a function of operating mode, thus stimulating the incorporation of the ambipolar drift term in the early point models. 


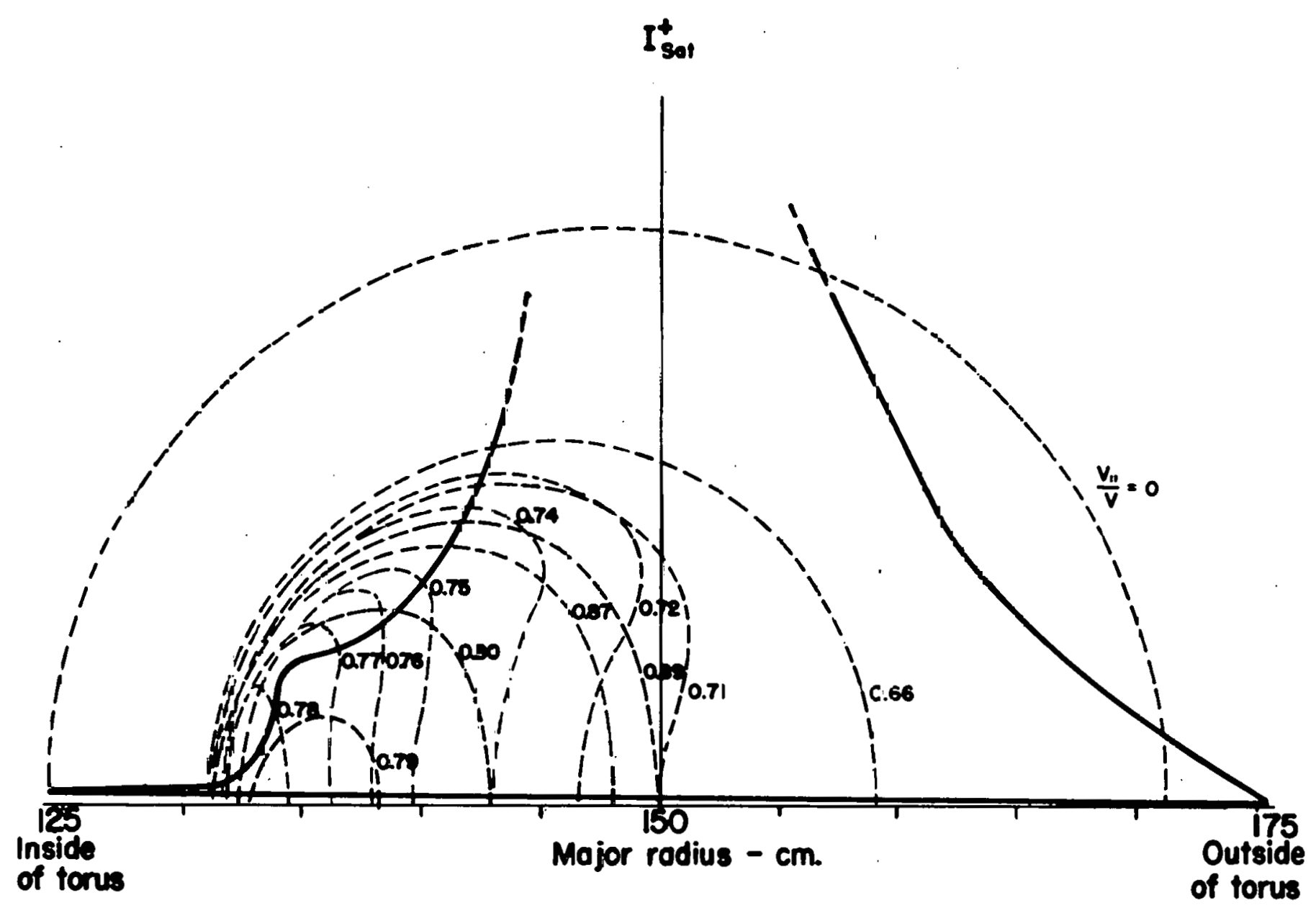

Fig. 8. Ion saturztion surrent measured with Lanjmuir probes as a function of radius. Dotted curves show closed drift surfaces for particles with various values of $\mathrm{v}_{\|} \cdot \mathrm{v}$ in the midpiane. 


\section{STABILITY OF THE ELECTRON ANNULUS}

The electron annulus plasma component in EBT was observed to be macrostable when the ambient neutral density was sufficiently high. A curve typical of early measurements of the stable decay of the diamagnetism following interruption of the applied power is shown in Fig. 9, in this case indicating a decay time of $\sim 1 / 4 \mathrm{sec}$. As the ambient neutral density was lowered, a point was reached in which fast spikes were noted in the diamagnetic signal corresponding to randomly occurring instability of the electron annuli. These fluctuations occurred independently in the various mirror sections. A comprehensive experimental examination of the stability boundaries and conditions required for stability has not yet been undertaken, but the behavior described above is consistent with the requirements of appreciable cold plasma density present for annulus stability ( $n_{\text {cold }} / n_{\text {hot }} \geq 1$ ). A significant fact is that stability of the annular plasma was observed even at low magnetic fields when the pressure profile peaked close to the toroidal axis (see Fig. 7). Under these conditions, the flux lines through the annulus region do not intercept a wal1. Thus, except for the outer edges of the annular plasma which could contain only a small fraction of the stored energy, "line-tying" to metal surfaces as a mechanism for stabilization must be ruled out. It is sufficient to remark here that no contradiction with theoretically predicted behavior was noted experimentally. 


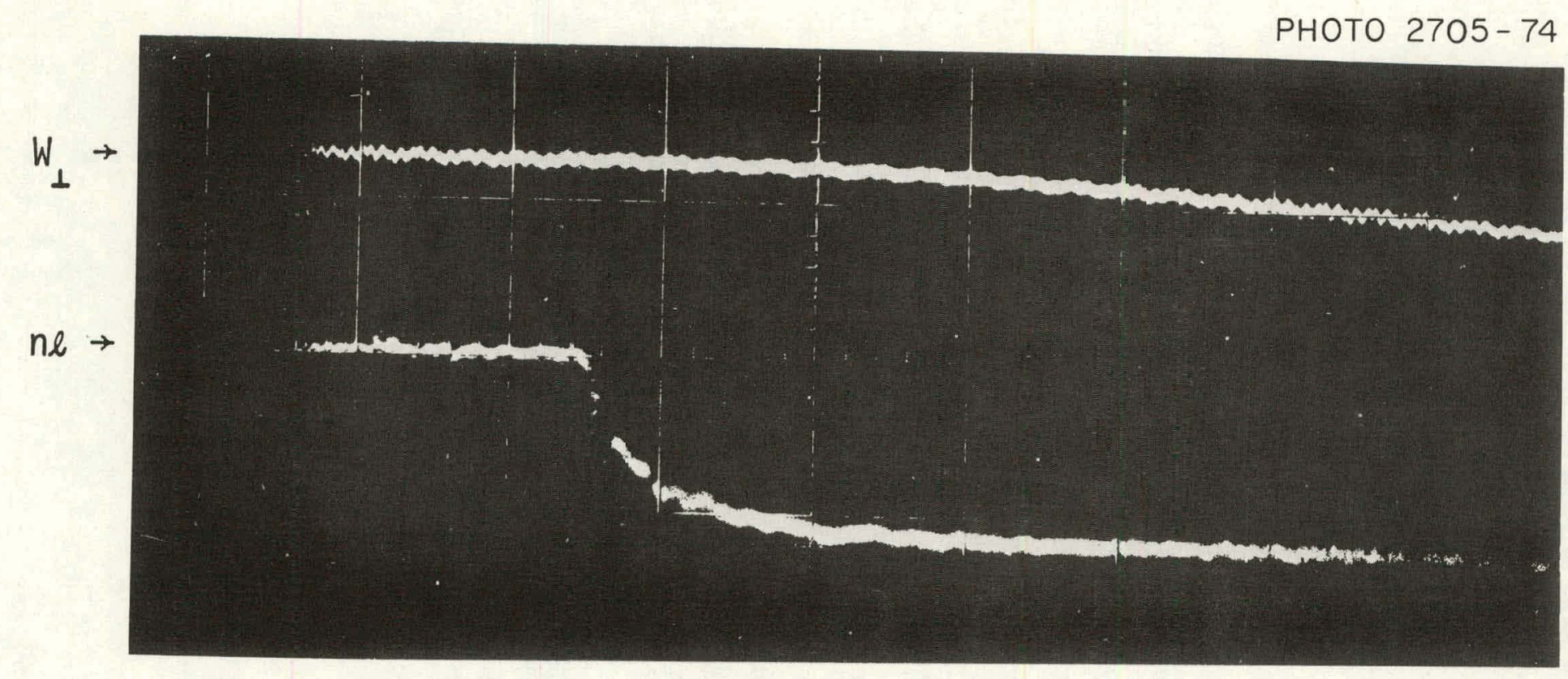

\section{$50 \mathrm{msec} / \mathrm{cm}$}

Fig. 9. Tracing of decays of $W_{\perp}$ (measured with diamagnetic 1oop) and $\mathrm{n}_{\mathrm{e}}$ (measured with interferometer) following turnoff of microwave power; the horizontal scale is $50 \mathrm{msec} / \mathrm{cm}$. 


\section{MEASUREMENT OF PLASMA PARAMETERS IN T-MODE}

\subsection{ELECTRON DENSITY}

The line density of electrons was measured using a 70-GHz microwave interferometer which incorporated a servo-driven, linear phase shifter which was used to null the bridge, thus yielding a precise analog output which can be continuously displayed. The plasma diameter is taken from the Langmuir probe data (Fig. 8) which indicated $\ell \cong 20-30 \mathrm{~cm}$. From the observed phase shifts in T-mode, $\mathrm{nl}=2-5 \times 10^{13} \mathrm{~cm}^{-2}$.

As we describe below, the density could also be deduced from bremsstrahlung measurements. These measurements yielded densities somewhat lower than the values quoted above, but because the temperature profile was certainly not flat and the energy range available for measurement was limited, this result is not surprising. In discussing the behavior of the toroidal plasma as a function of power or pressure, we use the relative density from bremsstrahlung observations normalized at the maximum values observed with both diagnostics.

\subsection{ELECTRON TEMPERATURE}

Two techniques were used to establish the electron temperature in the region of closed drift surfaces during operation of EBT in the stable T-mode. An experiment was carried out to detect Thomson scattered light at $90^{\circ}$ using a $1-\mathrm{J}$, repetitively pulsed ruby laser as a light source. The receiver optics and geometry are shown in Fig. 10. Data were obtained when using only $20 \mathrm{~kW}$ of heating power at $18 \mathrm{GHz}$. At the limited plasma density $\left(\mathrm{n}_{e} i 10^{12} \mathrm{~cm}^{-3}\right.$ ) and in the presence of a large plasma light background signal, it was difficult to obtain data over a broad range of operations. However, the experiment was valuable in establishing the existence of a warm electron component with $\mathrm{T}_{\mathrm{e}} \sim 150 \mathrm{eV}$.

The principle diagnostic tool used for electron temperature determinations was a "windowless" lithium-drifted, silicon barrier detector [Si(Li)] which was collimated to observe the bremsstrahlung radiation from the toroidal electron distribution. The detector geometry is shown in 
ORNL/OWG $75-18281$

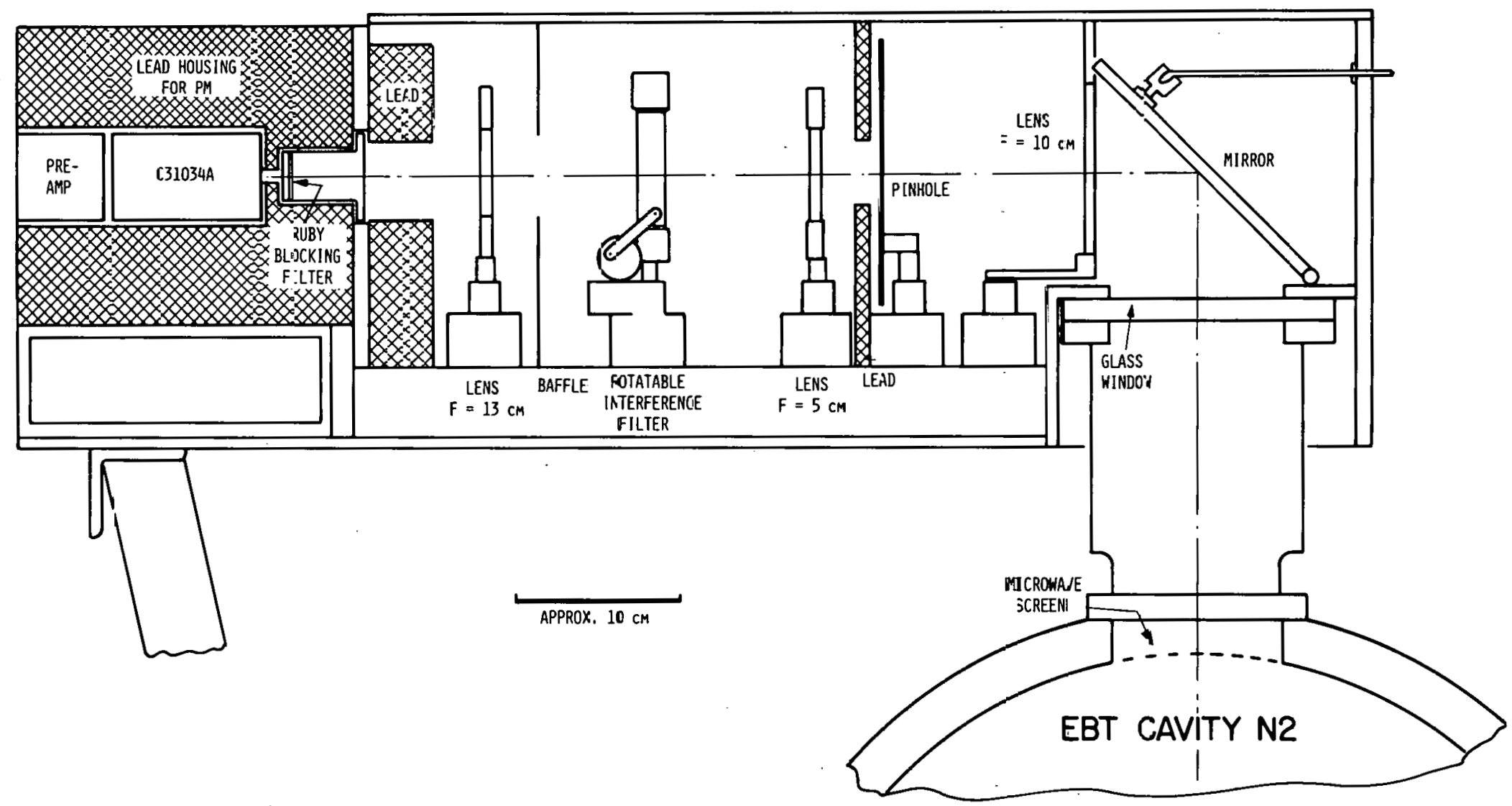

Fig. 10. Receiver optics for Thomson scattering measurement of ruby laser light at $90^{\circ}$. 
Fig. 11. A typical count rate vs energy spectrum (Fig. 12) illustrates the complications which had to be circumvented to establish the electron temperature. Since the anticipated temperatures were below $500 \mathrm{eV}$, it was necessary to subtract the continuum produced by the energetic electrons in the annulus and the aluminum $\mathrm{K}_{\alpha} \mathrm{x}$-ray line at $1.5 \mathrm{keV}$. The exponential decrease in photon intensity with energy and the modest electron temperature constrained the critical range of useful information to lie between 500 and $1200 \mathrm{eV}$. In this range, the efficiency of the detector was strongly influenced by material which condensed on the liquid-nitrogen cooled detector face. Efficiency calibration of the system in this range was carried out in place by operating EBT so that the energetic annulus was produced, but no appreciable toroidal plasma could be present. This was accomplished by energizing only those magnetic field coils in the mirror section viewed by the detector. The known continuum shape at low photon energies produced by the energetic electrons constituted an ideal radiation standard. The differences between observed count rate and those predicted from the observations at higher energy resulted in efficiency corrections which could be applied to the data when the facility was subsequently operated in T-mode. A typical reduced spectrum with $\mathrm{T}_{\mathrm{e}}=400 \mathrm{eV}$ is shown in Fig. 13. Using the formula for intensity [I(E)],

$I(E) d E=3.73 \times 10^{-21} \mathrm{n}_{e}^{2} \ell \mathrm{T}_{\mathrm{e}}^{-1 / 2} \mathrm{e}^{-\mathrm{E} / \mathrm{T}} \mathrm{edE}(\mathrm{keV} / \mathrm{sec})$

where $\mathrm{n}_{e}$ is in $\mathrm{cm}^{-3}, \ell$ is in $\mathrm{cm}$, and $\mathrm{E}$ and $\mathrm{T}$, are in $\mathrm{keV}$; $\ell$ is the length of plasma and the constant includes the geometric corrections for solid angle viewed. Both the density and electron temperature can be derived from the data. From observations with various operating conditions, the range of electron temperatures in EBT in T-mode lay between 150 and $600 \mathrm{eV}$.

\subsection{ION TEMPERATURE}

The ion temperature in EBT-I was determined by analysis of the energy spectrum of the energetic $H^{\circ}$ or $D^{\circ}$ atoms escaping from the 
ORNL/OWG/FED $\cdot 77352$

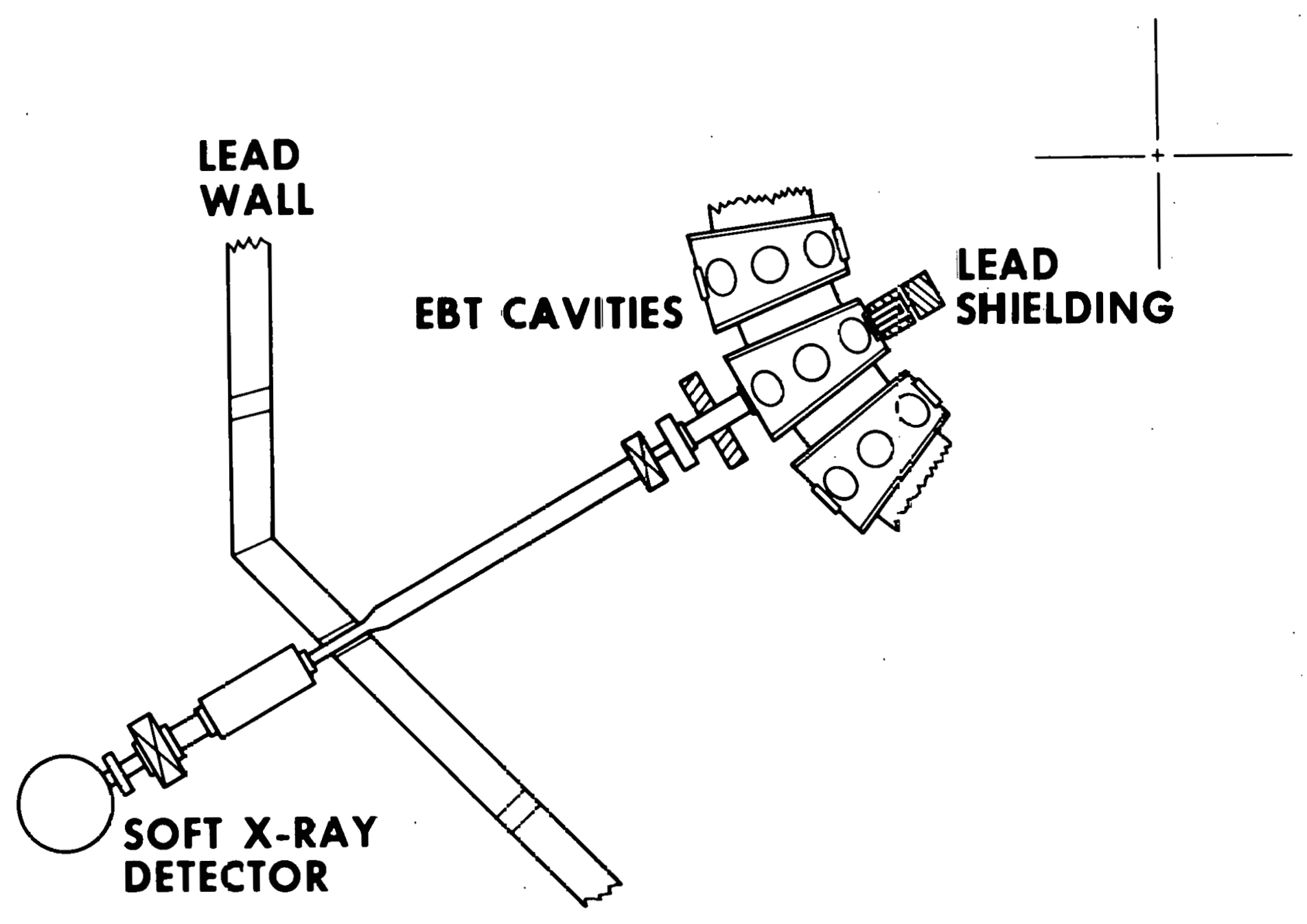

Fig. 11. Geometry used with Sị(Li) detector for viewing bremsstrahlung in EBT-I. 


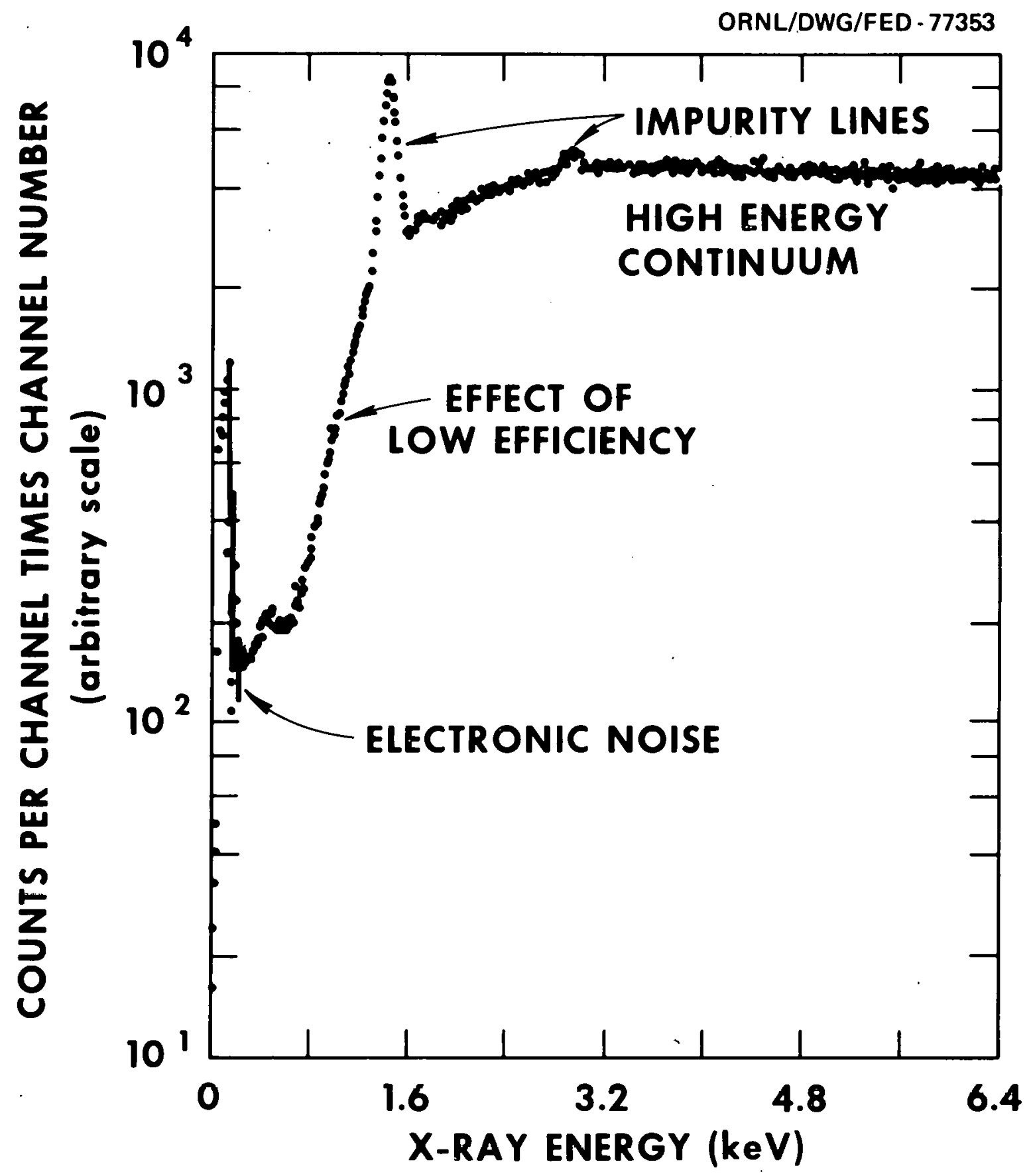

Fig. 12. Count rate spectrum obtained with Si(Li) detector collimated to view line through equatorial plane. 
ORNL / DWG / FED-78-430

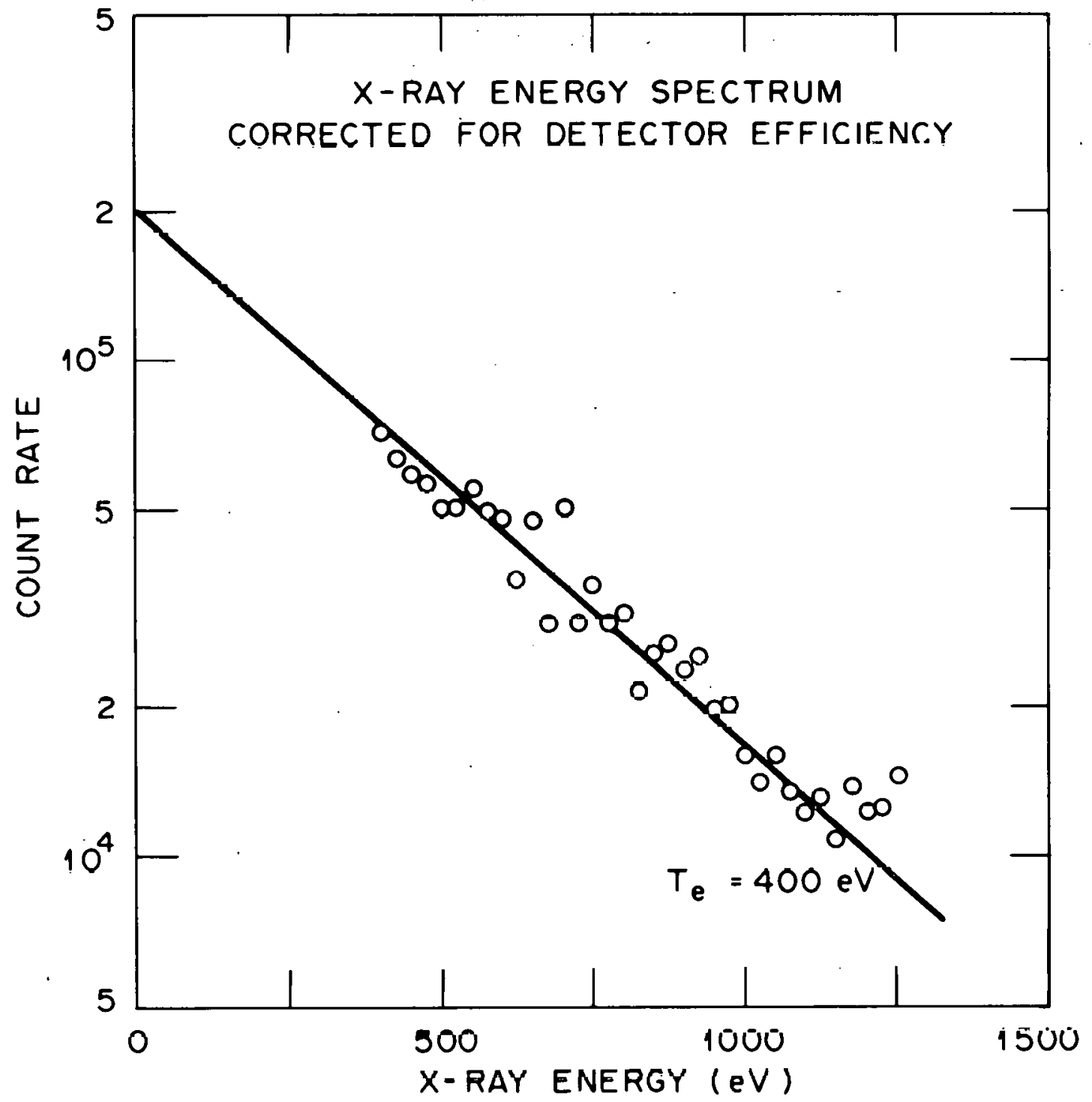

Fig. 13. Count rate distribution obtained with Si(Li) detector after correction for efficiency. 
plasma as a result of charge-exchange collisions between plasma ions and background neutral gas. Four different charge-exchange neutral spectrometers were used on EBT-I, each one sampling neutral particles escaping perpendicular to the toroidal magnetic field in the midplane of a toroidal bump. Energy analysis on all the spectrometers was accomplished by converting the neutral particles to ions, sweeping the voltage on a $45^{\circ}$ parallel-plate electrostatic energy analyzer, and then detecting the ions with a channel electron multiplier. Three of the spectrometers employed a differentially pumped nitrogen stripping cell to convert the neutral particles to positive ions; the fourth spectrometer used a cesium charge-exchange $\operatorname{cell}^{2}$ to convert the escaping neutral particles to negative ions. One spectrometer employed a velocity filter for mass analysis of the escaping neutrals, and one spectrometer was a four-beam device sampling four different chords of the plasma.

The typical geometry used in the charge-exchange measurement is shown in Fig. 14, with the neutral particle conversion cell in this case being the cesium cell. Calibration of the entire system was carried out with a low energy neutral particle beam whose intensity was calibrated against an ion beam at the same energy using a secondary emission detector as the primary detector. The secondary emission coefficient for hydrogen ions and neutrals was compared in a still unpublished experiment by Barnett and the variation of this parameter with energy was included in a recent reevaluation of the data. The recalculated efficiency for the system, using the nitrogen and cesium cells, is shown in Fig. 15.

Typical spectra of charge-exchange neutrals for the same plasma measured with the cesium cell and nitrogen stripping cell, corrected for efficiency, are shown in Fig. 16. Analysis of the data using the cesium cell, which has larger apertures and much higher efficiency, particularly at the lower energies, indicates a higher average energy and lower $\overline{n_{i} n_{0} l}$ than obtained from the nitrogen cell data. In both cases, the spectra were non-Maxwellian, as one would have anticipated from nonuniform neutral density and ion temperature profiles. The anticipated cesium cell advantages as a consequence of the higher efficiencies at lower energy have not yet been fully exploited because of a disturbing residual background count rate produced by photoelectrons. Further, the cell efficiency 
DRNL/DWG/FED $\cdot 77901$

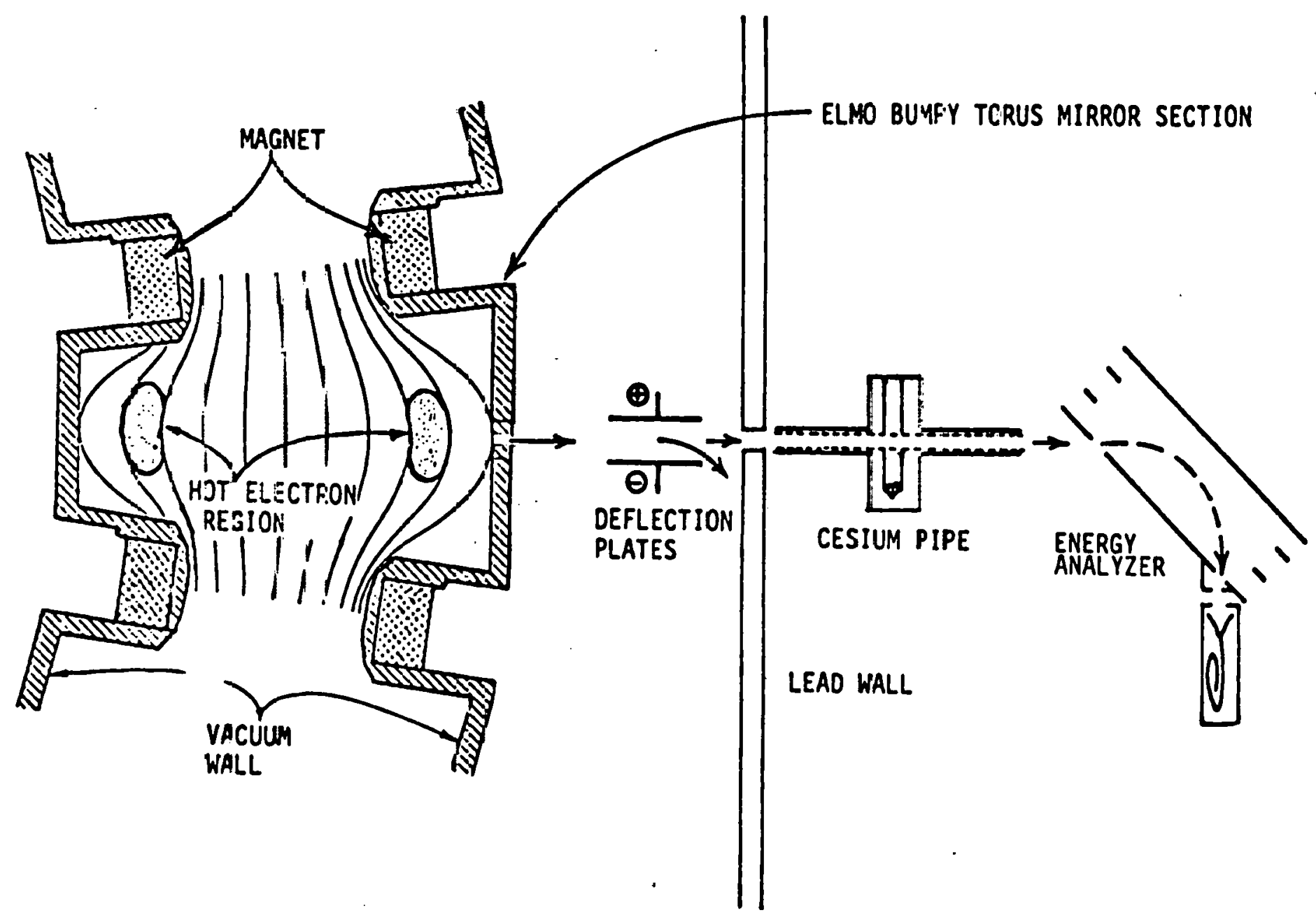

Fig. 14. Schematic arrangement of system used to detect chargeexahange neitral particles from EBT-I. The distance from the center of tre toroidal sector to the cesium cell reionizer is approzimately $2.5 \mathrm{~m}$. 


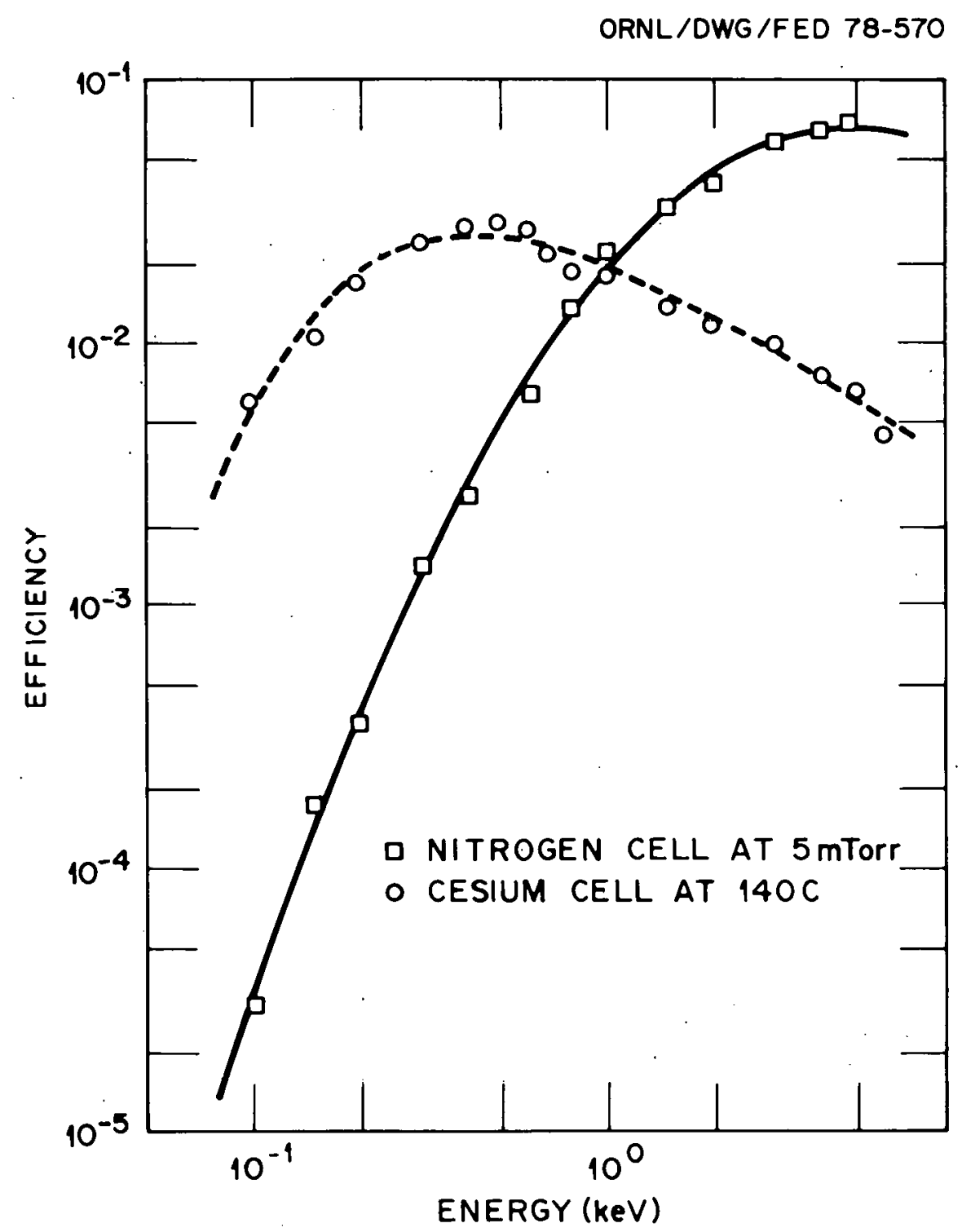

Fig. 15. Efficiency of nitrogen stripping cell and cesium heat pipe cliarge-exchange ccll ac a function of incident neutral particle energy in geometry used for ion temperalure ineasurements. 

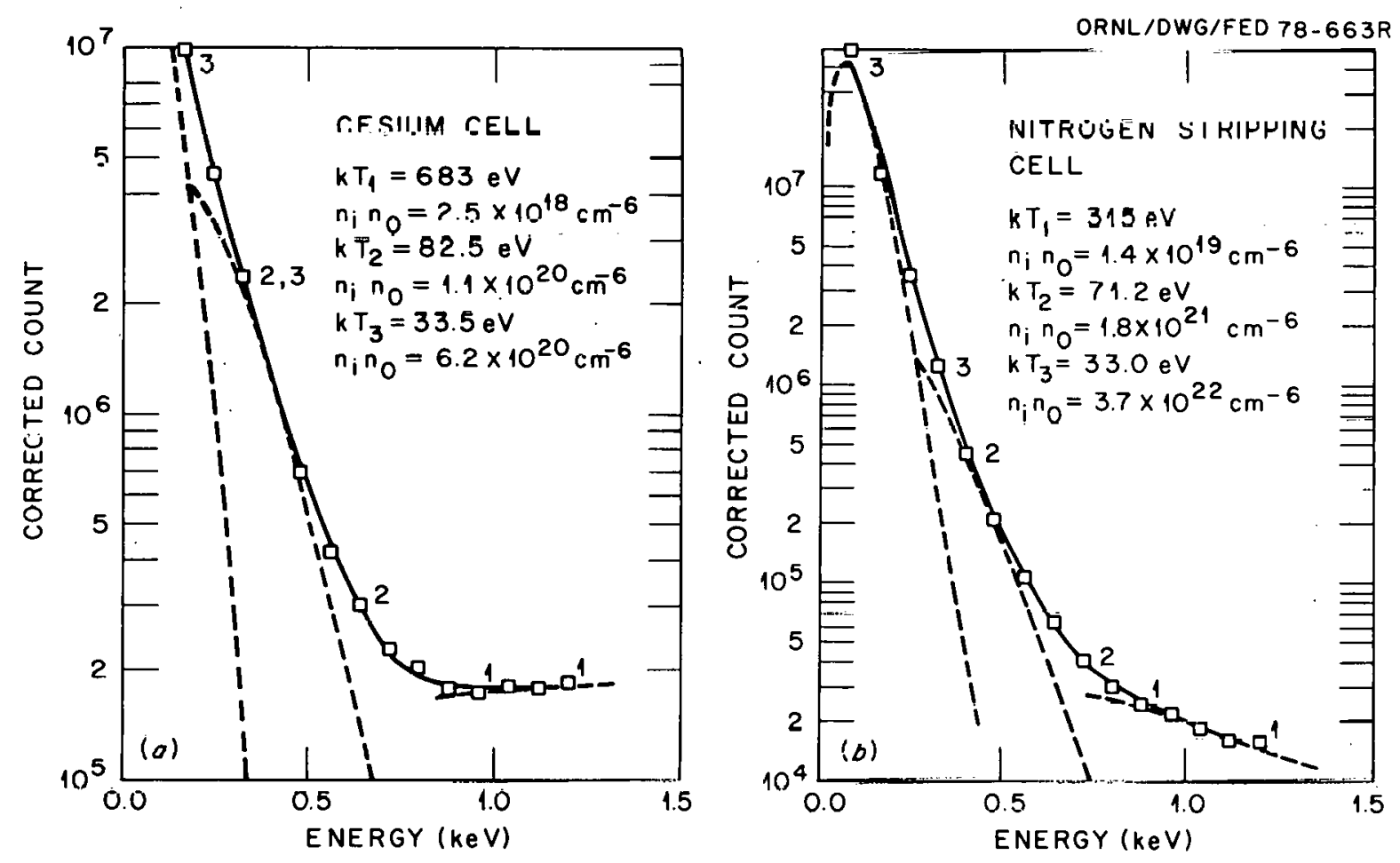

Fig. 16. Typical count rate spectra of neutral particle energy obtalned with nitrogen (a) and cesium (b) cell spectrometers under T-mode conditions. 
may be somewhat questionable at the lower energies because the calibration beam diameter was considerably smaller than the aperture size, leading to a possible overestimate of cell efficiency because of uncompensated scattering in the cell for uncentered particles. These uncertainties are expected to be resolved in future experiments.

Ion temperature measurements with both analyzers were made over a range of operating conditions in EBT-I. Data were taken over the range from C-mode through many combinations of pressure and power in $\mathrm{T}$-mode to the T-M mode transition. Central ion temperatures in $T$-mode ranged from $\sim 30 \mathrm{eV}$ near the transition between $\mathrm{C}$ - and T-modes to $\sim 150 \mathrm{eV}$ at the lowest pressure.

The value of $\overline{n_{i} n_{0} l}$ which is derived from the spectrum is of significance since it establishes whether the bulk of the ion population is observed (e.g., as contrasted with a possible ion runaway population). The value obtained from analysis of the nitrogen stripping cell data, $\overline{n_{i} n_{0} l} \sim 1.2 \times 10^{22} \mathrm{~cm}^{-5}$, is somewhat lower than spectroscopic estimates of $\mathrm{n}_{\mathrm{o}} \mathrm{n}_{\mathrm{e}}$ at the plasma center if one assumes that the hottest ions are confined nearer to the axis with $\ell \simeq 10 \mathrm{~cm}$. However, the nonuniform temperature profile introduces an additional uncertainty in the analysis which can only be removed by spatially resolved measurements of the neutral flux.

\subsection{SPACE POTENTIAL MEASUREMENT}

Confinement of plasma by the toroidal EBT geometry differs fundamentally from that in tokamaks because of the absence of a poloidal magnetic field component. In EBT the unequal radial fluxes of ions and electrons generate a self-consistent radial electric field which controls the ambipolar diffusion rate, whereas, in tokamaks the unequal radial particle fluxes drive a toroidal current which performs the same function. Therefore, it was of fundamental importance to the understanding of plasma confinement in EBT that the space potential be measured as a function of position.

R. L. Hickok and colleagues ${ }^{3}$ developed a technique for measuring space potential using a heavy ion beam probe. The energy of singly lonlzed heavy ions entering a plasma with space potential $\phi_{S}$ is reduced by $e \phi_{S}$. 
Doubly ionized ions produced by collisions with electrons in the plasma emerge with energy $2 \mathrm{e}_{\mathrm{s}}$ greater. The difference in energy between the injected beam energy and secondaries is $e_{s} \phi$. The ion orbits could be calculated precisely and those secondary ions which enter the detector traced back to a locus of points in the plasma. The intersection of these points with the beam path established the point in space where the secondary ionization took place (Fig. 17). By varying the beam energy and incident angle, the entire two-dimensional space in the midplane could be Lraced out - thus producing a map of the electric field in the plasma. The rather ronsiderablc adaptation of tlis technique to the steadystate mode and plasma parameter regime of EBT is depicted in the overall ion beam system (Fig. 18). The ion beam (generally $\mathrm{Rb}^{+}$, although $\mathrm{K}^{+}$and $\mathrm{Na}^{+}$were sometimes used) was generated and accelerated by a gun structure mounted above the midplane of a cavity in EBT. The beam passed through the sweep chamber and could be swept across the plasma (in the plane of the figure) or along the plasma (out of the plane of the figure). To minimize noise problems, the beam was usually chopped by driving the beam out of the plane with a high voltage pulse on the chop plates, and synchronous detection was used.

In practice the energy difference between singly and doubly ionized particles was measured uslng a parallel plate electrostatic elleigy analyzer. Small changes in energy which occur due to secondary ionization at positions where $e \phi_{S} \neq 0$ result in a deflection of the particles in the analyzer from their initially-centered position and the voltage change un the cop plate required to recenter them was proportional to this energy difference. Calibration of the system was provided by nulling the acceleration voltage against the voltage on the analyzer required to center primary beam ions directed onto the detector system and then using precisinn resistors to divide the analyzer voltage by precisely a factor of two for measuring potentials using $z=2^{+}$ions; similar precision resistor ratios were used for higher charge states. Closed loop feedback of the andlyzer voltage tracked the beam for changes of the accelerator gun voltage using narrow-band phase-sensitive detection at the beam chopping frequency to maximize the signal/noise ratio and accuracy. 


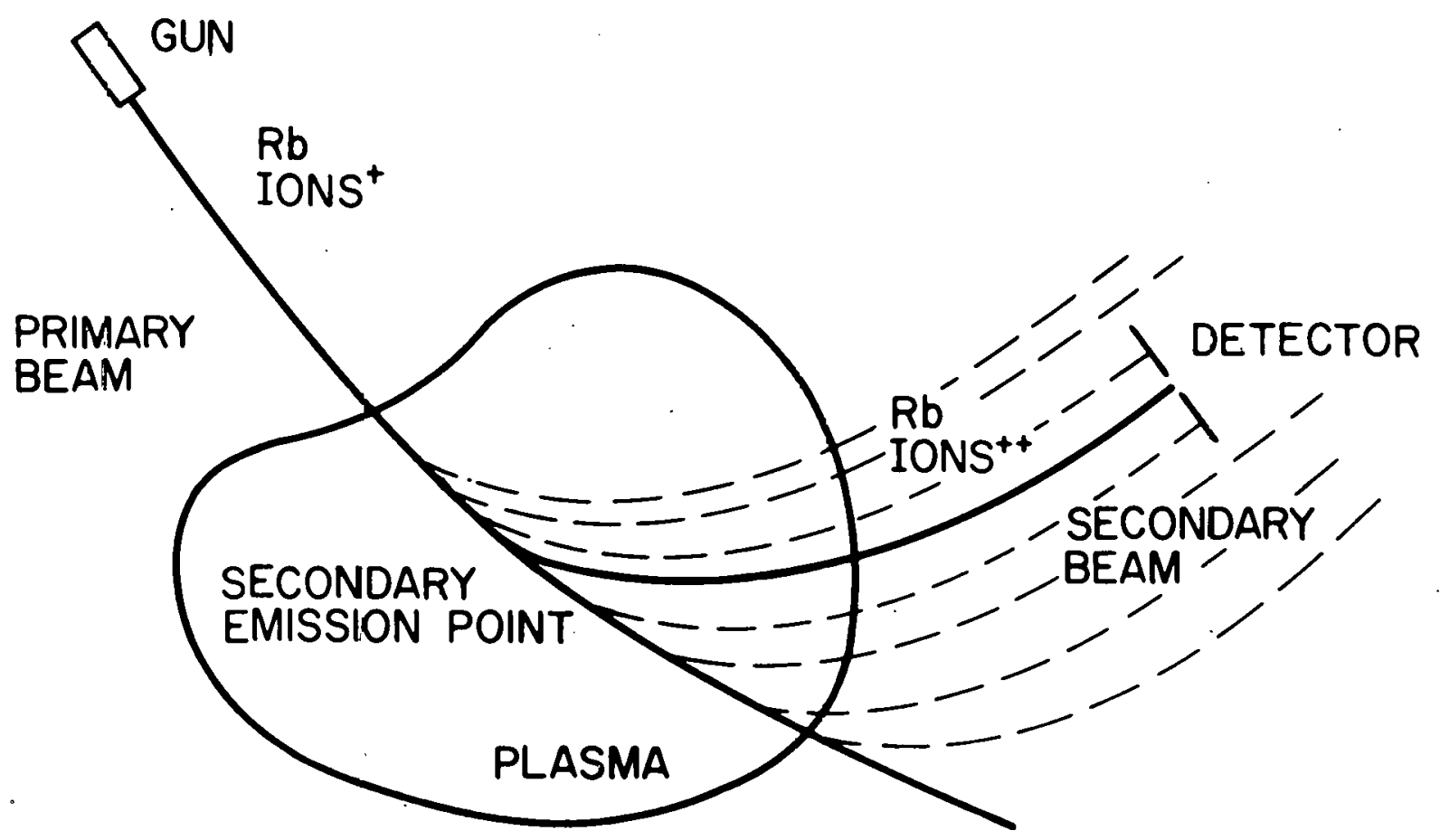

Fig. 17. Schematic drawing showing path of injected $\mathrm{Rb}^{+}$ions and orbits of secondarily ionized $\mathrm{Rb}^{++}$ions from various points along beam path. 


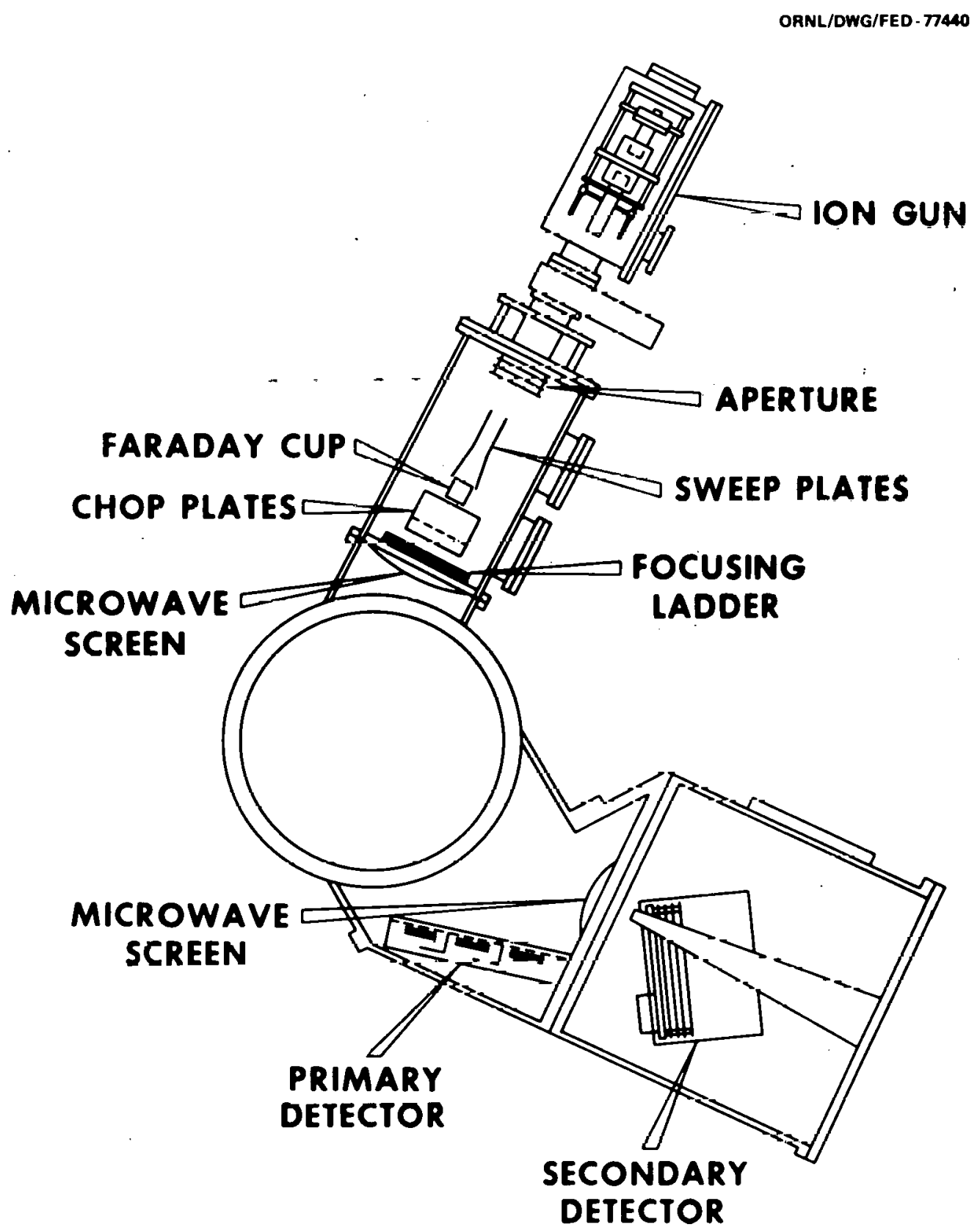

Fig. 18. General layout of heavy-ion beam probe system used in EBT-I. Secondary detector is a graded-fleld, parallel plate energy anlalyzer. 
The result of analysis of a typical scan with variable beam energy and incident angle with the plasma in the stable $\mathrm{T}$-mode is shown as a potential map in Fig. 19. Under optimum confinement conditions, the ambipolar electric field was nearly symmetric about a point $4 \mathrm{~cm}$ inside the toroidal axis, pointed radially inward, and was of sufficient magnitude to strongly influence the poloidal drifts. The symmetry shown in Fig. 19 was critically dependent on error field global nulling (see Sect. 4.7) and having T-mode conditions. For example, in the $\mathrm{C}$-mode, the scans were qualitatively different (Fig. 20); the potentials were smaller, the electric field was reversed, and the symmetry was significantly reduced.

\subsection{IMPURITIES}

Radiation in the visible and vacuum uv was measured in order to determine the density of impurities and neutral hydrogen and to establish their spatial distributions. A 2.2-m Rowland circle grazing incidence spectrometer was used to measure resonance line radiation down to $\$ 50 \AA$. A $0.125-m$ Ebert spectrometer was used in the uv $(1200<\lambda<2300 \AA)$ with two scanning mirrors (Fig. 21) to study the principal line radiation from carbon and aluminum as a function of spatial position. Analysis of this data was carried out using an iterative algebraic reconstruction technique (ART) similar to methods developed for medical tomography, ${ }^{4}$ which permitted reconstruction of the light emission distribution even for asymmetric cases. Finally, a $0.5-\mathrm{m}$ Ebert spectrometer was used in the visible region to continue the aluminum and carbon studies and to look at the ratio of line intensities from $\mathrm{He}^{0}$ to measure the electron temperature in those regions in which this technique was relevant.

To determine the impurity densities from the observed light intensities, the electron density and temperature spatial distributions must be known. As established from the Langmuir probe measurements, other than the annulus, the EBT plasma consisted of two spatially separated electron distributions. These components differed in both energy and density as a consequence of their different confinement properties. The toroidal plasma, near the axis of the device, was confined on closed drift surfaces, and its confinement time was therefore controlled by a radial diffusive transport rate out 

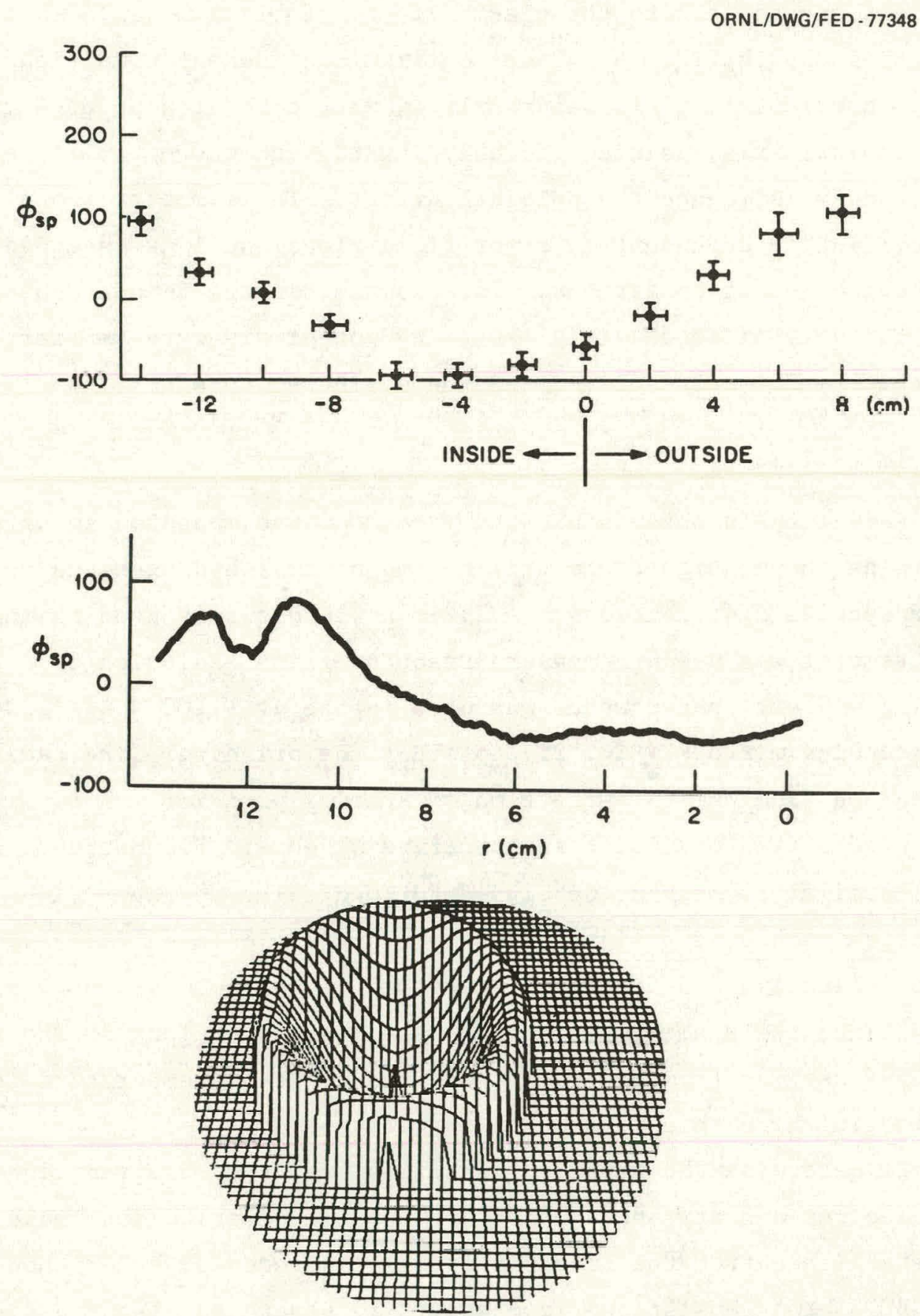

Fig. 19. Space potential measured in EBT-I as a function of position in the midplane (T-mode conditions). The computer-generated perspective view includes data taken at various energies and angles of beam incidence and then "spline-fits" the data to present the smoothed display. No data is taken outside the points where the maximum potentials are found. 

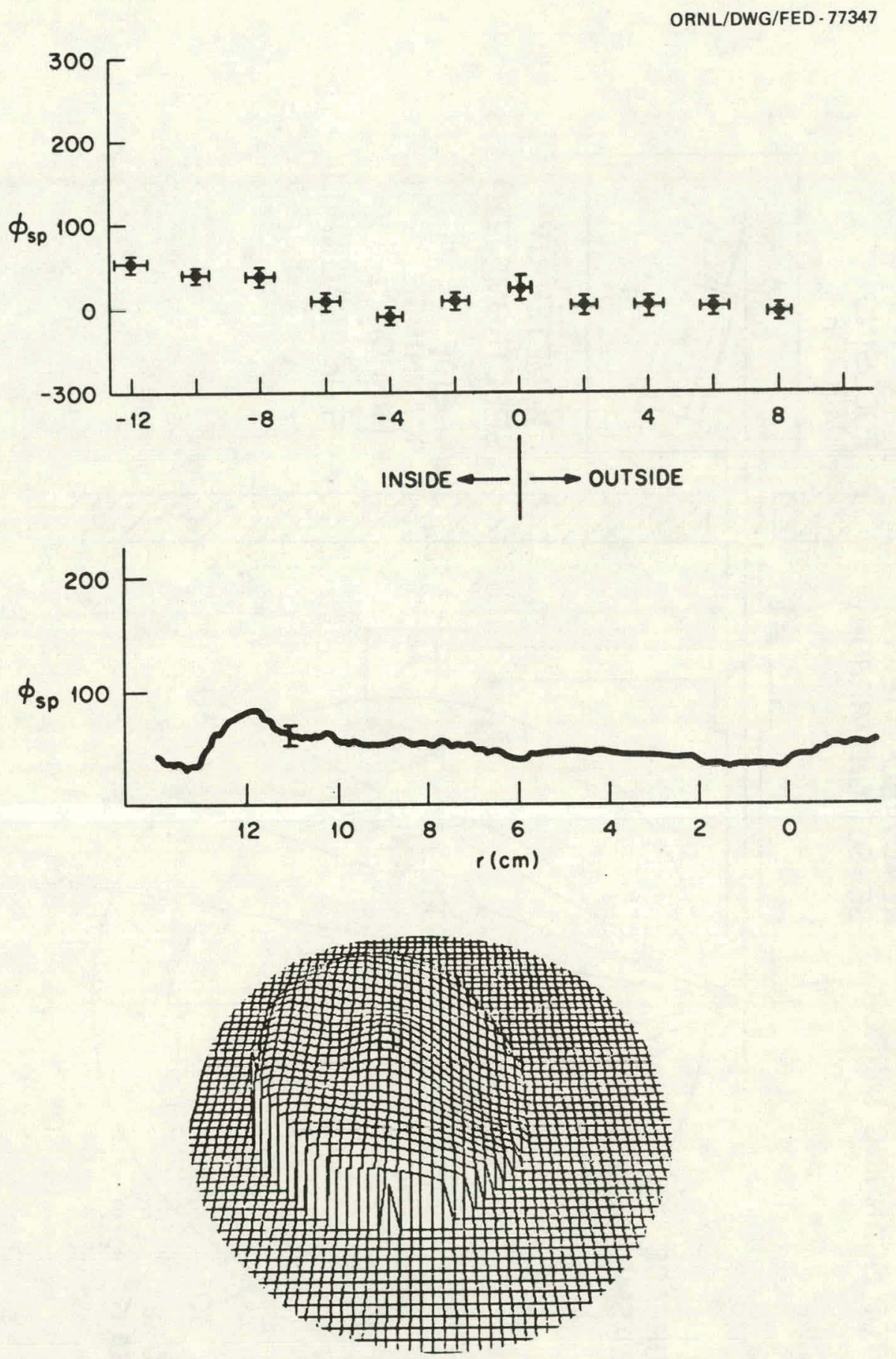

Fig. 20. Space potential measured in EBT-I as a function of position in midplane (C-mode conditions). 


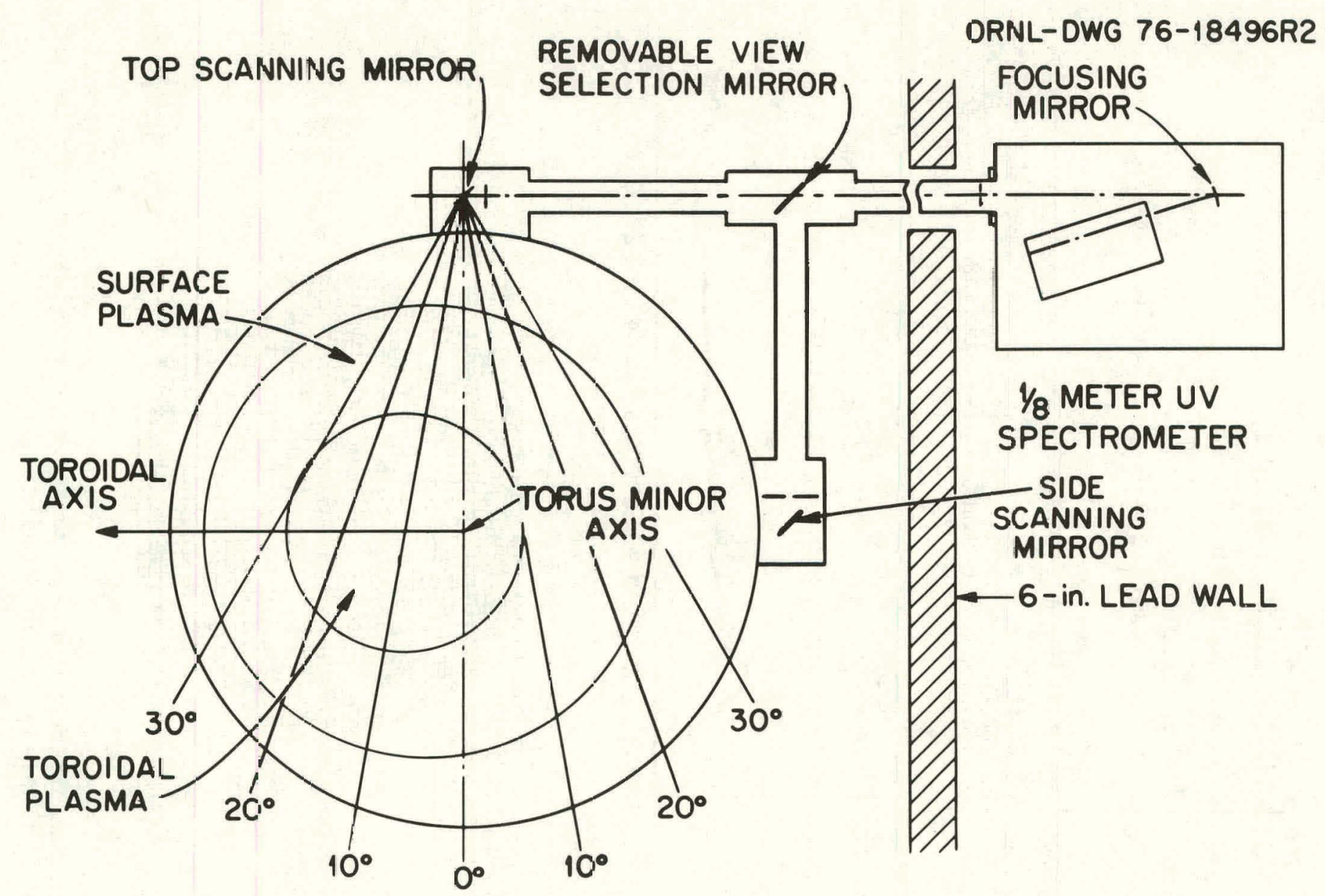

Fig. 21. Geometry used for spatial scanning of uv light from plasma in the micplare of EBT-I. Distance $\exists$ long light path to spectrometer focussing mirror is $22.75 \mathrm{~m}$. 
to flux lines which ultimately intercept the vacuum walls. In contrast, the plasma outside the radius of the annulus was confined on drift surfaces which contained flux lines which intersected the vacuum walls. Thus the confinement time of these plasma particles was controlled by their poloidal precession rate onto these open flux lines and the diffusion time in velocity space which permitted them to attain sufficient $v_{\|} / v$ to escape the mirror trap.

The measurements showed that in the stable T-mode, the surface plasma had an electron temperature of $\sim 30 \mathrm{eV}$ and an average density of i $3 \times 10^{11} \mathrm{~cm}^{-3}$. Impurity ions were also found in the toroidal plasma, although for all species the density was observed to be lower than that seen in the surface plasma.

The densities observed at various radii in the surface plasma, multiplied by the electron density, are listed in Table 2 for a typical stable T-mode operation. The rate equation at equilibrium for a particular charge state, $j$, is

$$
\begin{aligned}
\frac{d n}{d t}=0=n_{j-1} n_{e}\langle\sigma v\rangle_{j-1}^{j} & +n_{j+1} n_{e}\langle\sigma v\rangle_{j+1}^{j}-n_{j} n_{e}\langle\sigma v\rangle_{j}^{j+1} \\
& -n_{j} n_{e}\langle\sigma v\rangle_{j}^{j-1}-\frac{n_{j}}{\tau},
\end{aligned}
$$

where $\langle\sigma v\rangle_{j}^{j+1}$ is the ionization rate coefficient from state $j$ to $j+1$, $\langle\sigma v\rangle_{j}^{j-1}$ is the recombination rate coefficient, and $\tau_{j}$ is the ion confinement time in state $j$. Neglecting recombination for the densities and temperatures in EBT-I, the value $n_{e} \tau_{j}$ could be calculated from measured values of the ratio $n_{e} n_{j} / n_{e} n_{j-1}$. The values $n_{e}{ }^{\tau}$ obtained are also listed in rable 2 . Tahle 3 1i.sts the impurity ion densities near the center of the plasma.

These figures imply confinement times of considerably less than 1 msec for the impurity ions in the surface plasma. In effect, the surface plasma acted as a divertor to ionize incoming neutral impurities and direct them to the walls before they diffuse into the toroidal plasma where they 
Table 2. Product of electron density with carbon ion density $\left(n_{e} n_{j}\right)$ and ion lifetime $\left(n_{\ell} \tau_{j}\right)$ in the surface plasma region of EBT

\begin{tabular}{llll}
\hline Species & Radius & $n_{e^{n}} n_{j}\left(\mathrm{~cm}^{-6}\right)$ & $n_{e^{\tau} j\left(\mathrm{~cm}^{-3} \mathrm{sec}\right)}$ \\
\hline C II & 10 & $0.61 \times 10^{20}$ & \\
\multirow{2}{*}{ C III } & 15 & $0.46 \times 10^{20}$ & \\
& 10 & $0.51 \times 10^{20}$ & $3.2 \times 10^{7}$ \\
C IV & 15 & $0.36 \times 10^{20}$ & $3.2 \times 10^{7}$ \\
& 10 & $0.49 \times 10^{19}$ & $4.4 \times 10^{7}$ \\
\hline
\end{tabular}


Table 3. Impurity ion densities in the region of the toroidally confined plasma $\left(\mathrm{n}_{\mathrm{e}}=1.5 \times 10^{12} \mathrm{~cm}^{-3}\right)$

\begin{tabular}{cc}
\hline Ion charge state & Density $\left(\mathrm{cm}^{-3}\right)$ at $\mathrm{r}=0$ \\
\hline C II & $5.9 \times 10^{7}$ \\
C III & $3.9 \times 10^{7}$ \\
C IV & $1.0 \times 10^{7}$ \\
C V & $<1.4 \times 10^{7}$ \\
AI II & $4.7 \times 10^{7}$ \\
Al III & $1.3 \times 10^{8}$ \\
\hline
\end{tabular}


could affect confinement. The divertor effect is enhanced by the presence of the large positive potential barrier observed at the position of the electron annulus (Fig. 19) which must be overcome by impurity ion heating if impurities were to reach the toroidal plasma. In the short lifetime of the impurity ions, such heating could not be accounted for by classical processes.

To illustrate the spatial reconstruction technique, in Fig. 22 we show the neutral aluminum emissivity. The deep "hole" in the profile illustrates the effectiveness of the surface plasma in ionizing incoming neutral atoms. In Fig. 23 we show the reconstructed 1ight emission profile from singly ionized aluminum. The peak at the center could not be accounted for from the neutral particle source in Fig. 22. The explanation for the Al II peak is that sputtered aluminum atoms from the walls in the mirror throats which were not seen in the midplane penetrated the toroidal plasma as neutrals and were then ionized. Excitation of these ions followed as they streamed along flux lines to the midplane, and the subsequent deexcitation light was seen by the spectrometer.

\subsection{NEUTRAL HYDROGEN}

Neutral hydrogen plays an important role in the plasma equilibrium because it is the source of particles which sustained the plasma against losses to the walls. Spectroscopic measurements of light helped to determine the neutral density distribution of hydrogen and the average hydrogen atom velocity. The latter measurement was particularly significant since the mean free path for neutral penetration, $\lambda$, depends on the atomic velocity as

$\lambda=\frac{\mathrm{v}_{\mathrm{o}}}{\mathrm{n}_{\mathrm{e}}\left(\langle\sigma v\rangle_{\text {ion }}+\langle\sigma v\rangle_{\mathrm{cx}}\right)}$,

where $v_{o}$ is the atom velocity, $\langle\sigma v\rangle_{i o n}$ is the ionization rate coefficient, and $\langle\sigma v\rangle_{c x}$ is the charge-exchange rate coefficient averaged over the particle velocity distributions. Particle losses out of the plasma center must be balanced by ionization so that 

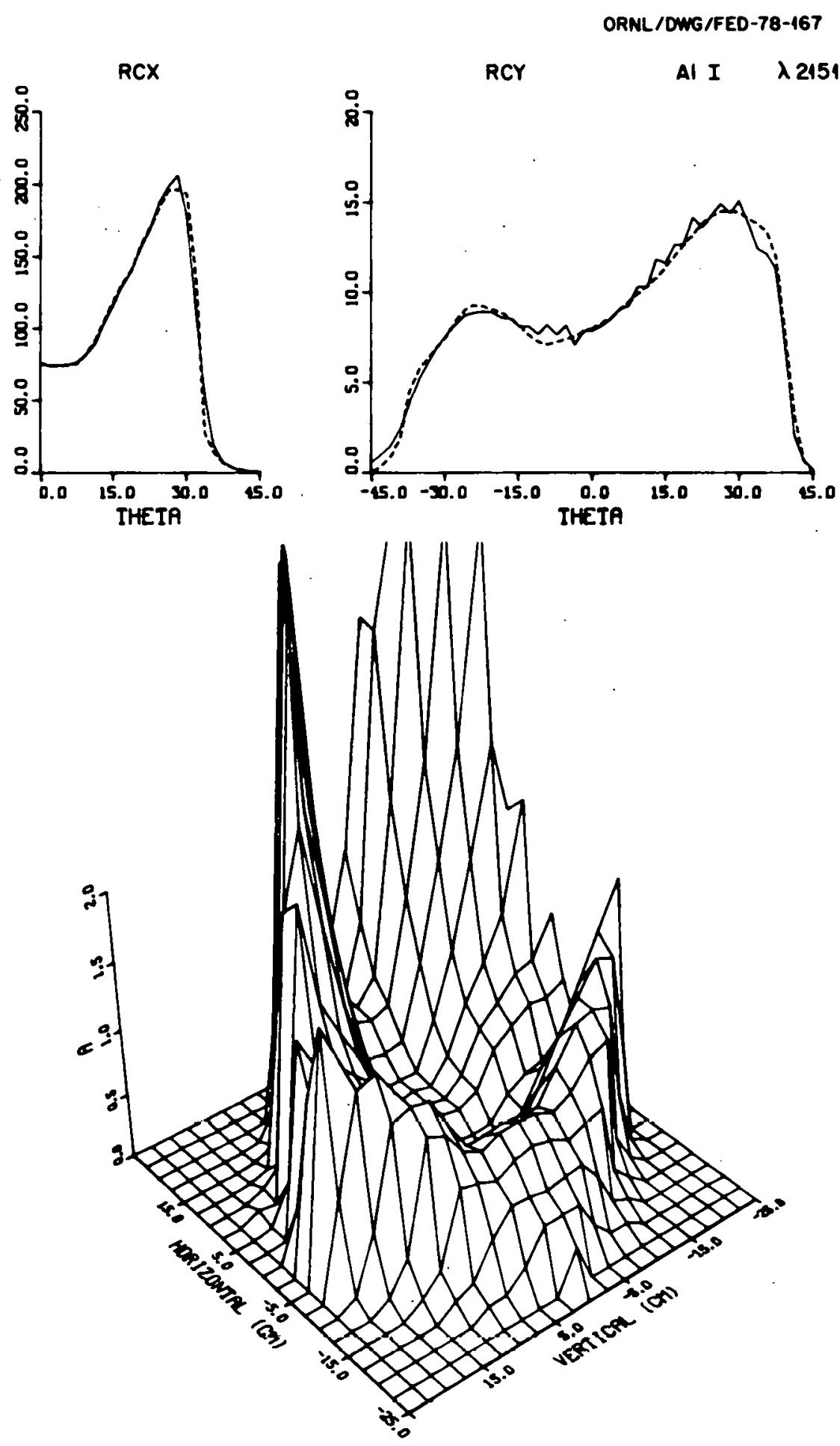

Fig. 22. Light intensities from $A l$ I at $\lambda=2151 \AA$ as a function of angle of view $(\theta)$ from top (RCY) and side (RCX) ports (dotted line observed solid line - reconstructed). Lower drawing is reconstructed emission distribution in midplane calculated using iterative algebraic reconstruction technique. 

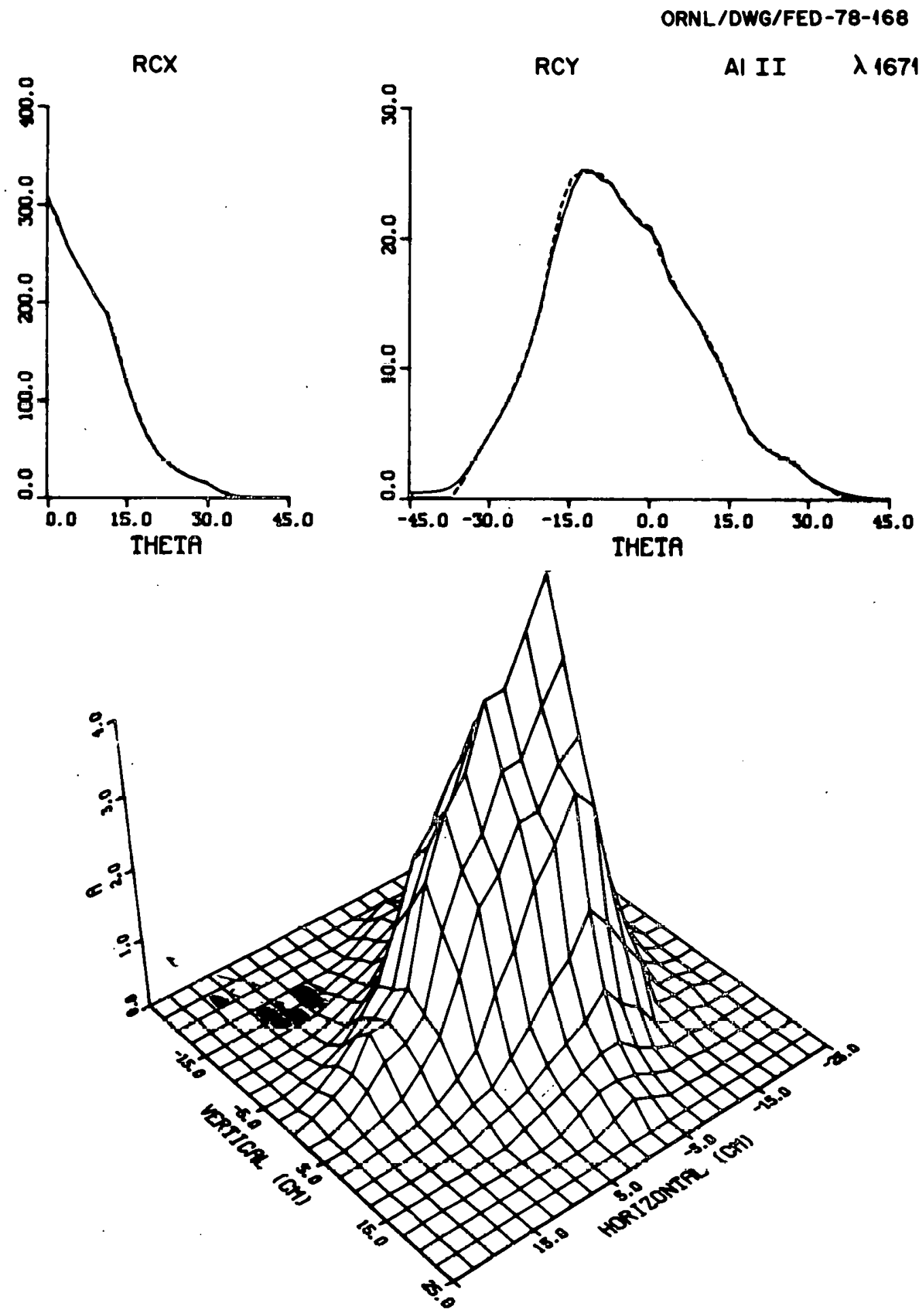

Fig. 23. Light intensities from A1 II at $\lambda=1671 \AA$ and reconstructed emission distribution in the midplane calculated using iterative algebraic reconstruction technique. 
$\frac{n_{e}}{\tau}=n_{e} n_{0}\langle\sigma v\rangle_{\text {ion }}$.

As we pointed out in Sect. 3, for stability of the annulus, it was necessary that the cold plasma density (and, therefore, neutral density for its support) in the surface plasma be relatively high. On the other hand, for long confinement time, $n_{o}$ at the plasma center must be low. The ratio of $n_{0}$ (center) to $n_{0}$ (surface) was strongly influenced by the neutral velocity since the mean free path was of the same order of magnitude as the plasma radius.

By measuring the line shape of emitted $H_{\alpha}$ light at $\lambda=6563 \AA$ using a high resolution Fabry-Perot interferometer, it was possible to determine the mean velocity of excited atoms. The observed profile (Fig. 24) is made up of several fine structure lines which yielded an estimated mean energy of $0.5 \mathrm{eV}$, when Doppler spread was applied to each of them and the result compared with the observation. This energy is considerably lower than if one assumed that the neutral particles entered the vacuum volume initially as molecules, which would be dissociated in the surface plasma producing a flux of hydrogen atoms with a mean energy of $\sim 5 \mathrm{eV}$.

The relatively low atom energy observed can be understood by recognizing that these atoms recirculated between the plasma and the wall many times before being pumped by the diffusion pumps. As a consequence, the measurement implied that atoms were not recombined into molecules in the time they spent on the plasma wall. 'l'he details of the chemistry of the hydrogen and the relatively pure surface aluminum metal are not well known, but the low recombination rate implied that atoms were relatively immobile in the metal lattice. This conclusion was substantiated by the large amounts of hydrogen which eventually diffused out of the walls after the experimental day was terminated, the amount continuing for a period as long as several hours if the experiment were operated for a long time. The low atom velocity (and consequent short mean free path) resulted in a nonuniform atomic density profile in EBT. However, without additional information on the electron density profile, the reconstructed light 


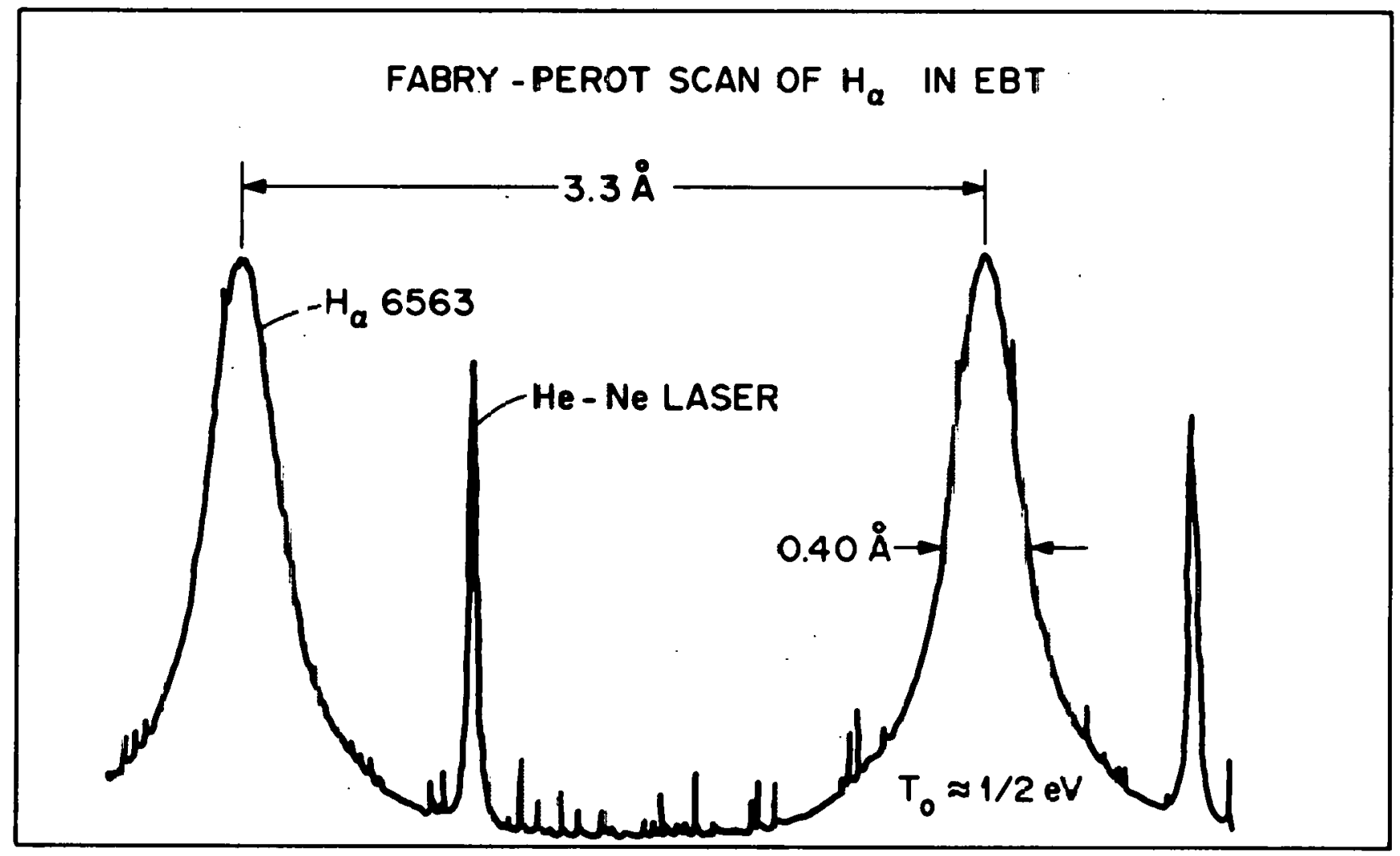

Fig. 24. Wavelength scan of $\mathrm{H}_{\alpha}$ light using Fabry-Perot interferometer. For resolution comparison the light from a HeNe laser is also siown. 
emission profile [at Lyman- $\alpha$ wavelengths for example (see Fig. 25)] could not be simply correlated with the detailed spatial distribution of hydrogen. It was possible to estimate $n_{0}(r=0)$, the hydrogen density at the center of the plasma, however, using the absolute calibration of the mirrors and spectrometer carried out at the Johns Hopkins University and the peak electron density measured in the toroidal plasma $n_{e}=2 \times 10^{12}$ $\mathrm{cm}^{-3}$ ). It is also important to note that the mirror reflectivities (the mirrors are MgF coated for high reflectivity in the uv) were found to change over a short period of time when exposed to the plasma. To alleviate this problem, the mirrors were rotated so they did not see the plasma unless they were being used to take data. In spite of this precaution, when the mirror reflectivities were recalibrated after the data run they were found to have decreased by a factor of 25. ' During the last few runs at which data were taken, under similar plasma conditions, the count rates were reproducible, and for this reason the last calibration was used in establishing absolute numbers. The value obtained from scans in the stable T-mode was $\mathrm{n}_{0}(\mathrm{r}=0) \cong 1 \times 10^{10} \mathrm{~cm}^{-3}$, which is at least a factor of two higher than would be expected from power balance estimates (see Sect. 5). The iterative reconstruction technique used. in the analysis could overestimate the emission in the center, but it would be surprising if the amount were greater than a factor of two. More work is needed to resolve this apparent difference.

From the observed light distribution, assuming the electron density in the surface plasma region is approximately $3 \times 10^{11} \mathrm{~cm}^{-3}$, we estimated the neutral density in this region as $\mathrm{n}_{0} \sim 5 \times 10^{10} \mathrm{~cm}^{-3}$, again a factor of two or more higher than anticipated.

\subsection{TOROIDAL CURRENTS AND FIELD ERROR EFFECTS ON EBT}

Magnetic perturbations in closed field confinement systems can cause field lines to spiral out of the confinement regions and lead to enhanced plasma losses, instabilities, and anomalous convection. ${ }^{5}$ Even though the inherent field errors in EBT were relatively small, their effects on the plasma behavior could have been deleterious because of the long lifetime of the ring-stabilized plasma and the absence of toroidal currents. 

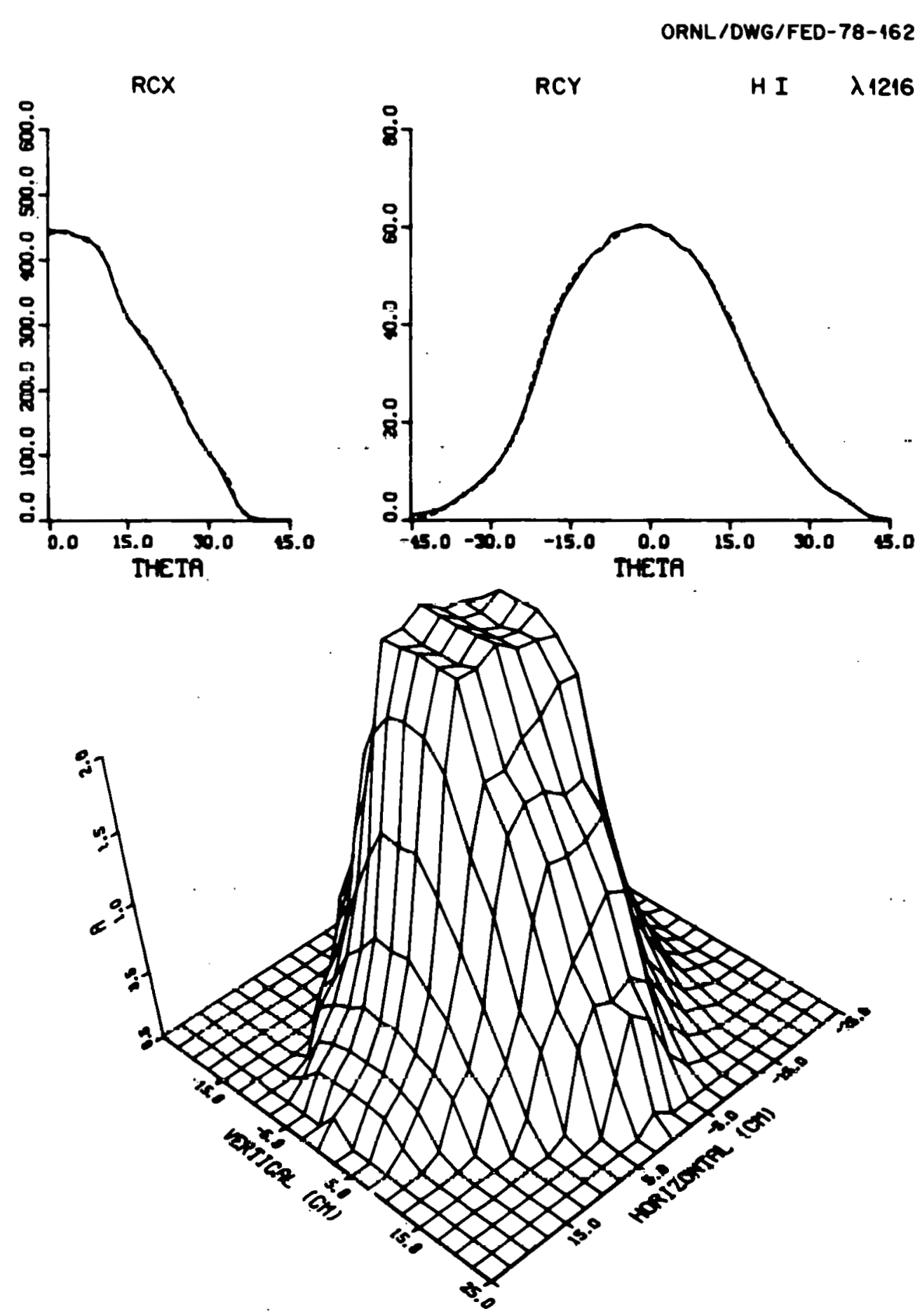

Fig. 25. Light emission profiles from hydrogen atoms (H I) at $\lambda=1216 \AA$ (see Fig. 22 for definitions). 
The vacuum field error was measured in EBT using an electron beam probing technique. The estimated total field error was

$\frac{\Delta \mathrm{B}}{\mathrm{B}} \cong \frac{\Delta \mathrm{r}}{2 \pi \mathrm{R}} \leqslant 1.5 \times 10^{-4}$

where $\Delta r$ is the displacement of field lines about the "unperturbed". positions and $R$ is the major radius; measurements were taken for $B \cong$ $0.2 \mathrm{~T}$. The measured total field error included contributions from the earth's magnetic field ( $20 \%$ in $\Delta B$ ), local perturbations, and electron drifts. The field errors resulting from imperfections in coil winding, bus bars, ferromagnetic materials, etc., were found to be quite small (by orders of magnitude) compared with those in many other closed field confinement devices because of the simple coil geometry and careful design and construction. In order to minimize the $\mathrm{n}=0, \mathrm{~m}=1$ component error field, two pairs of current loops were wound around the torus as schematically shown in $\mathrm{Fig}$. 26. The loops were about $50 \mathrm{~cm}$ from and parallel to the minor axis; each loop consisted of a 20-turn coil capable of carrying a current up to $30 \mathrm{~A}$. The transverse magnetic field generated by these loops could be used to cancel the average error field or to increase the field error, thus permitting studies of plasma stability and confinement with the error field as a parameter.

The plasma was found to exhibit a small net toroidal current $(\leq 100 \mathrm{~A})$ related to the field error effect. This toroidal current was experimentally monitored by two toroidal coils surrounding the torus (see Fig. 26); the resulting measurements are shown in Fig. 27. The effect of magnetic field error cancellation is evident in this figure from the large reduction of toroidal current. The residual global error was taken to be zero at the minimum toroidal current; thus, the original error field $\Delta B_{0}$ could be estimated from the current required for null toroidal current.

$$
\frac{\Delta B_{o}}{B} \cong-\Delta B_{1} \cong \frac{I_{c}}{2 \pi r B} \cong 3 \times 10^{-4}
$$


10.5 GHZ (LORH) MICROWAVE FEED (1)

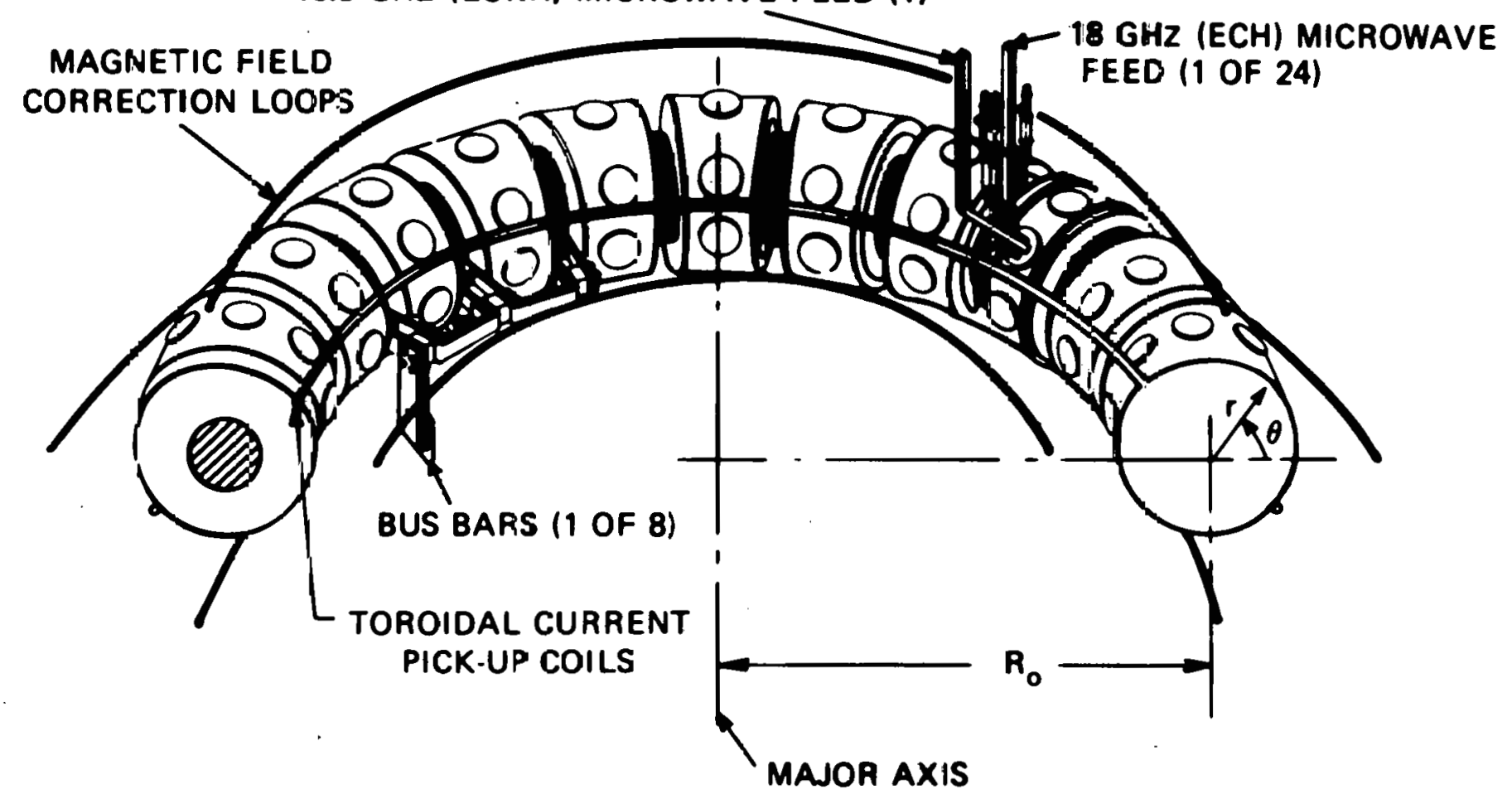

Fig. 26. Schematia section of EBT-I showing locatzans of quadripolar correction coils and toroidal current pick-up loof. 


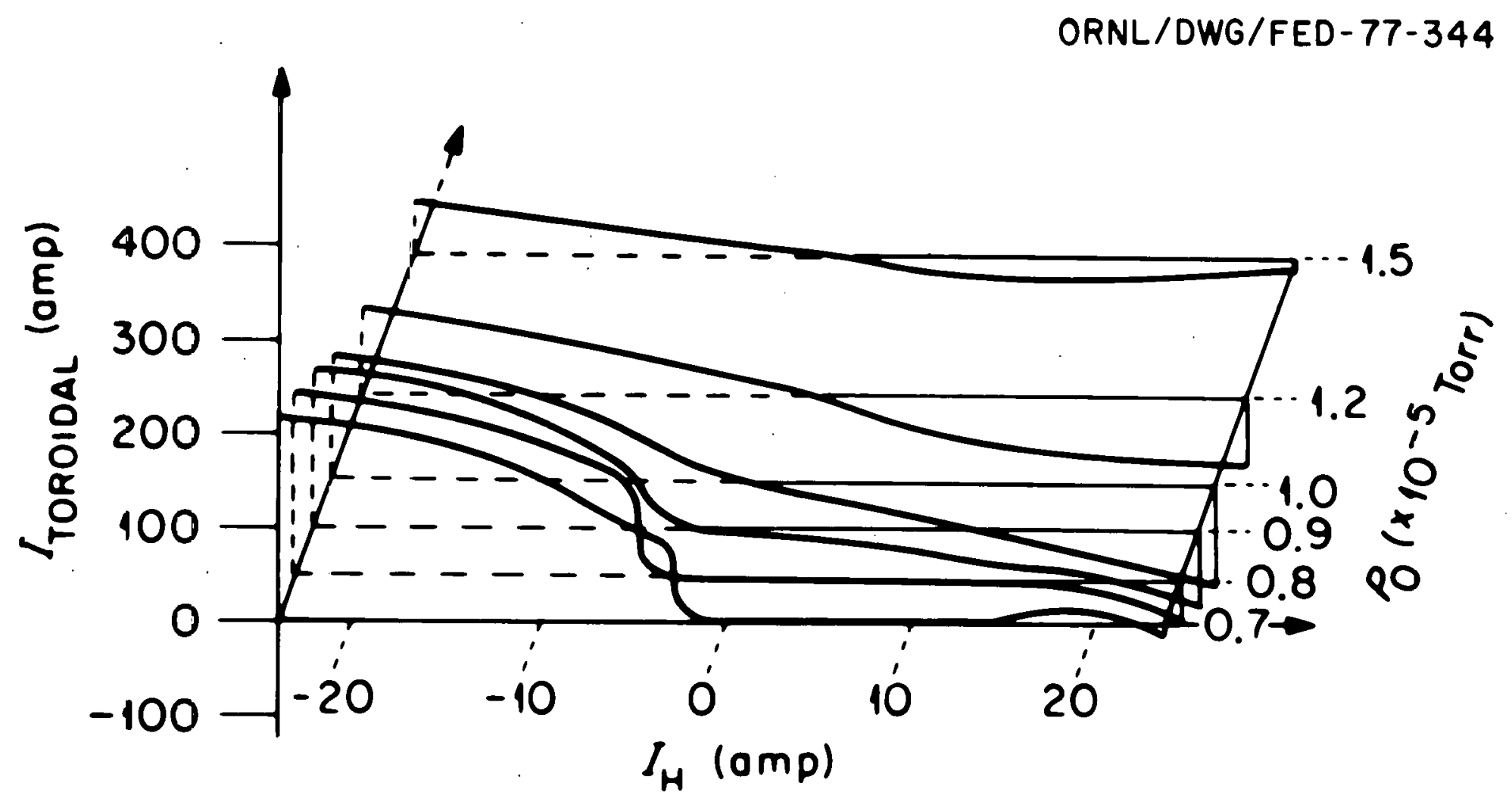

Fig. 27. Toroidal current measured with pick-up loop as a function of horizontal error field current for plasmas produced at various pressures. 
which is close to the integral vacuum field error measured by the electron beam probe.

Assuming that the error field produces an additional radial component to the otherwise axisymmetric bumpy magnetic field, a toroidal current in the plasma will be driven by the component of radial electric field lying along the magnetic flux lines.

$j_{\|}=\frac{E_{\|}}{n_{\|}}=\frac{E_{r}}{n_{\|}} \frac{\Delta B_{r}}{B}$,

where $\eta_{\|}$is the resistivity $\eta_{\|}=\sqrt{m_{e} / 2 \pi}\left(\mathrm{ze}^{2} / 32 \varepsilon_{0}\right)\left[\ln \Lambda /\left(\mathrm{kT}_{\mathrm{e}}\right)^{3 / 2}\right]$. Integrating $j_{\|}$across the plasma cross section yields the toroidal current

$I=\int j_{\|} d \theta$

$$
\begin{array}{r}
\int_{0}^{a} r d r \int_{-\pi / 2}^{\pi / 2} d \theta \frac{E_{1}(r)}{2 \pi n_{\|}(r)}\left(\frac{1}{R_{0}+r \cos \theta}-\frac{1}{R_{0}-r \cos \theta}\right) \Delta r \cos \left(\theta-\theta_{0}\right) \\
\cong \frac{1}{2}\left(\frac{a}{R}\right)\left(\pi a^{2}\right)\left\langle\frac{E_{1}}{n_{\|}}\right\rangle \frac{\Delta B}{B}
\end{array}
$$

where the directional cosine of the field error, $\cos \theta_{0}$, and the sign of current density $j_{\|}=j_{\|}(r, \theta)$ have been taken into account explicitly. Using a typical set of EBT plasma parameters, $\mathrm{T}_{e} \cong 200 \mathrm{eV}, \mathrm{E}_{\perp}(\mathrm{r}) \cong$ $\mathrm{kT}$ e/ea, and $\Delta B / B \cong 1 \times 10^{-4}, \mathrm{Eq}$. (8) gives a total toroidal current of $150 \mathrm{~A}$, which is in good qualitative agreement with measurements. Thus a strong relationship between field error cancellations and toroidal current magnitude could be inferred.

A more detailed comparison of the data in Fig. 27 with results of this model for the source of the observed toroidal current showed 
additional elements of agreement. At high pressures the observed current varied linearly with error field as predicted (Eq. 8). These conditions constituted C-mode states with highly collisional plasmas where the model should be quite applicable. At lower pressures ( $T$-mode) the plasma became less collisional, and the existence of annuli stabilized the toroidally confined component. The toroidal current was found to be significantiy reduced over a significant range of variation of $I_{H}$.

A tentative explanation for the observed low current has been suggested. Recognizing that the toroidal plasma was less collisional in the T-mode, most of the particles would be circulating on closed drift surfaces when field errors are small. Thus, very few charges would leave the toroidal column along the magnetic lines of force and Eq. (7) would not be applicable. The plasma would carry very little current until the field error becomes large enough so that an appreciable fraction of the drift surfaces intersect the wall. A simple calculation shows that this transition can occur within the range of field errors found experimentally; $\delta B / B \sim 0.6-1 \times 10^{-3}$.

The effects of magnetic field errors on plasma stability and confinement in EBT are demonstrated in Fig. 28 for plasma in the stable T-mode. The net toroidal current, the averaged plasma density, the averaged density fluctuation amplitude, and the plasma potential measured at the center and near the annulus are plotted together with the horizontal field error as functions of the current $I_{H}$ applied to the field correction coil. The correlation of the abrupt change in the plasma observations at a critical error field (in this case $I_{H} \sim-8 \mathrm{~A}$ ) is particularly striking. Within the range of error fields where the toroidal current was small $\left(-10 \mathrm{~A}<\mathrm{I}_{\mathrm{H}}<+15 \mathrm{~A}\right)$, the $\mathrm{T}$-mode properties agreed with those described in earlier sections (negative central potential, low plasma fluctuations, nl increase of $230 \%$ compared to that with large error fields present).

It is possible to evaluate the influence of error fields on neoclassical diffusion for sufficiently small values of error fields. If one adds to the radial velocity component in the usual drift kinetic equation the radial drift due to the error field, one obtains 


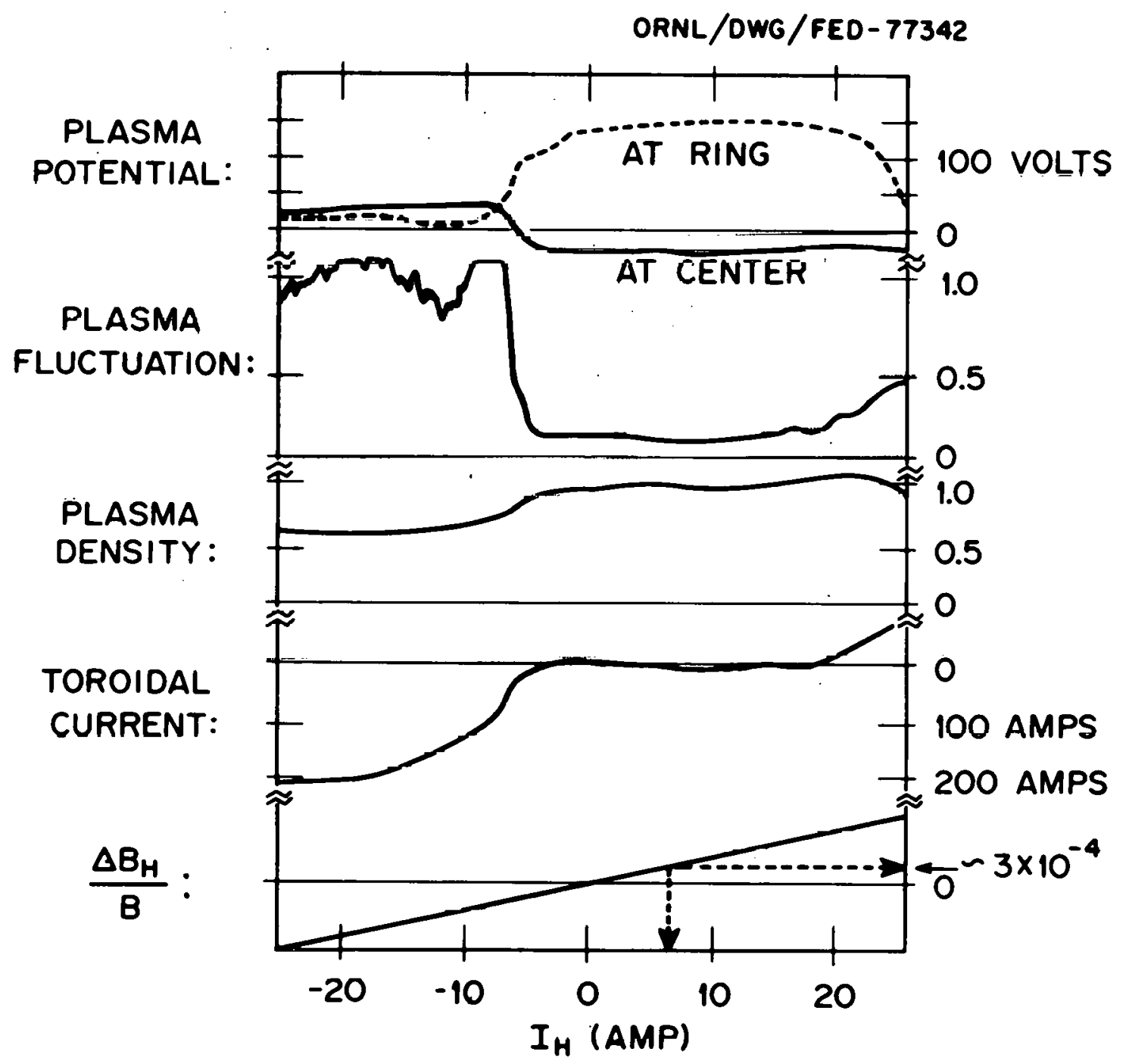

Fig. 28. Correlations of several parameters measured simultaneously as the horizontal field error scans in the range of $\pm 1-2 \times 10^{-3}$. The probable system global error is estimated to be $3 \times 10^{-4}$, as indicated by the arrowhead. 
$v_{r}=v_{o} \sin \theta+v_{\|} \frac{\Delta B_{r}}{B_{r}}$,

where $v_{0}$ is the vertical drift due to toroidal field curvature. Fourier analyzing and linearizing the drift kinetic equation leads to an expression for the electron distribution function, $f_{m n}$, which may be used to calculate the radial flux, $\Gamma_{r}$,

$\Gamma_{r}=\int \frac{\mathrm{d} \theta \mathrm{d} \phi}{(2 \pi)^{2}} \mathrm{f}_{1} v_{\mathrm{r}}$.

The new diffusion coefficient, $D^{*}$, can be evaluated using

$\Gamma_{r}=D^{*} \nabla_{r} n+\mu_{r}^{*} \mathrm{nE} r$

with $\mu^{*}=\mathrm{qD}^{*} / \mathrm{kT}_{\mathrm{e}}$.

One obtains for $D^{*}$

$$
D^{*} \cong \frac{V_{0}^{2}}{2} \frac{v}{v^{2}+\left(\frac{V_{D}}{r}\right)^{2}}+\frac{\left\langle V^{2}\right\rangle}{3} \sum_{m, n}\left(\frac{\Delta B_{m, n}}{B}\right)^{2} \frac{v}{v^{2}+\left(m \frac{V_{D}}{r}\right)^{2}+\left(n \frac{V_{\|}}{R}\right)^{2}} .
$$

The first term in Eq. (12) is the usual neoclassical diffusion ${ }^{6}$ rate due to toroidicity (asymmetry), while the second term is the result of the radial magnetic perturbation. ${ }^{7}$ We observe from Eq. (12) that for $\mathrm{n} \neq 0$ the magnetic field error effect on diffusion is negligible, and for 
the $\mathrm{m}=1, \mathrm{n}=0$ component the error field effect could be comparable to toroidicity only when

$$
\frac{\Delta \mathrm{B}}{\mathrm{B}} \geq\left(\frac{\Delta \mathrm{B}}{\mathrm{B}}\right)_{\mathrm{Cr}} \cong \sqrt{3 / 2} \frac{\rho}{\mathrm{R}} \sim 10^{-4}-10^{-3}
$$

for EBT-I plasmas. Field errors, therefore, simply modify the usual neoclassical diffusion rate by the factor

$\mathrm{D}^{*}=\mathrm{D}_{\text {neoclassical }}\left[1+\alpha\left(\frac{\mathrm{K}}{\rho} \frac{\Delta \mathrm{B}}{\mathrm{B}}\right)^{2}\right]$.

The value $\Delta B_{r} / B$ for which the diffusion coefficient is changed only negligibly by the error fields is inside the range of values of $\triangle B / B$ for which the toroidal currents were observed to be small - i.e., electron transport was likely unaffected in this range of $I_{H}$. Consistent with this fact was the relatively small variation of plasma properties between $-10 \mathrm{~A}$ and $+15 \mathrm{~A}$.

Outside this range, the abrupt change in parameters indicated a new mechanism was taking place. The high fluctuation level was probably a clue to the effect - perhaps toroidal current induced instabilities. These regimes have not been thoroughly explored, nor has the possibility of spatially localized toroidal currents, but future plans include these scudies. 


\section{DISCUSSION OF MEASUREMENTS}

\subsection{ESTIMATE OF TOROIDAL PLASMA CONFINEMENT TIME}

The measurements described in the previous section constitute the basis for a description of the plasma properties in EBT-I. Combining the results of the various diagnostics, we may describe a "typical" state in the macrostable T-mode of operation. Using $50 \mathrm{~kW}$ of resonant heating power at $18 \mathrm{GHz}$ and $6 \mathrm{~kW}$ of profile heating power at $10.6 \mathrm{GHz}$, distributed among the 24 toroidal sectors, with the magnetic field adjusted for $\mathrm{B}_{\mathrm{Oo}}=0.5 \mathrm{~T}$ and with ion gage readings of $\sim 4 \times 10^{-6}$ torr, a plasma with the following properties is produced:

$$
\begin{array}{rlrl}
\overline{\mathrm{n}}_{\mathrm{e}} & =1.5 \times 10^{12} \mathrm{~cm}^{-3} & \mathrm{~W}_{1} \text { (annulus) } & =40 \mathrm{~J} \\
\mathrm{~T}_{\mathrm{e}} & =400 \mathrm{eV} & \mathrm{a} & \cong 12 \mathrm{~cm} \\
\mathrm{~T}_{i} & =100 \mathrm{eV} & \overline{\mathrm{E}}_{\mathrm{r}} & =\frac{\Delta \phi_{\mathrm{s}}}{\mathrm{a}}=-16 \mathrm{~V} / \mathrm{cm}
\end{array}
$$

A near-circular hot electron annulus is genelated at $\bar{r}=15 \mathrm{~cm}$ with the center displaced inward $\sim 2.5 \mathrm{~cm}$. The total stored energy contained in each annulus is $40 \mathrm{~J}$ from integrating flux loop diamagnetic measurements.

We find that the plasma consists of a warm, toroidally-confined essentially Maxwellian distribution with a mean radius of $10-15 \mathrm{~cm}$, its center displaced toward the toroidal axis by $\sim 4 \mathrm{~cm}$, stabilized by the relatively high- $\beta$ ( $\beta \sim 15-20 \%$ ) electron annular plasma all surrounded by a cooler surface plasma (Fig. 29). The surface plasma properties are limited by its confinement time, which is relatively short since the drift surfaces in this region all hit the vacuum wall for $v_{\|} / v \geq 0.5$. This surface region acts as a divertor region for incoming neutral impurities beciause of its relatively high density $\left(\mathrm{n}_{\mathrm{e}} \geqslant 10^{11} \mathrm{~cm}^{-3}\right)$, electron temperature $\left(\mathrm{T}_{\mathrm{e}} \sim 30 \mathrm{eV}\right)$, and short confincment time.

These multiple plasma components are sustained in steady state by the applied microwave power. For purposes of extrapolation to devices with higher power and field, and especially for comparison with theoretical models, it is of interest to focus one's attention separately 


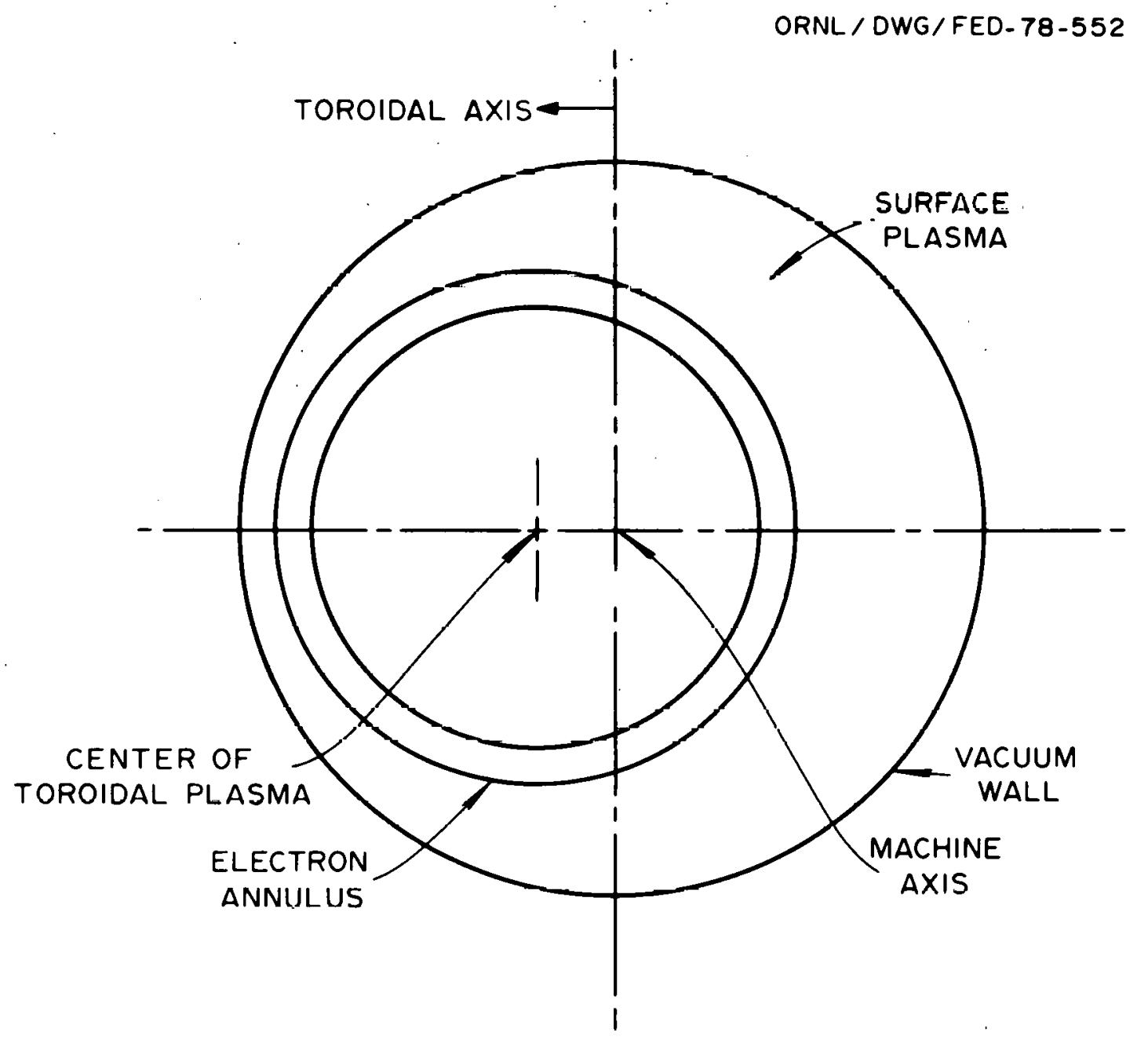

Fig. 29. Schematic representation of plasma components in midplane belwèn coils in Libil. 
on the toroidal plasma and its confinement. Experimentally, this is difficult to do because the power division among the various plasma components is not directly measurable. Rather, it is necessary to determine the amount of power required to sustain each plasma component from measurements of their respective lifetimes and energy. If the annulus power $\mathrm{P}_{\mathrm{A}}$ and surface plasma power $\mathrm{P}_{\mathrm{S}}$ are subtracted from the total microwave power $P_{\mu}$ it is possible to estimate the power required to sustain the toroidal plasma, $\mathrm{P}_{\text {toroidal }}$.

$\mathrm{P}_{\text {toroidal }}=(1-\alpha) \mathrm{P}_{\mu}-\mathrm{P}_{\mathrm{A}}-\mathrm{P}_{\mathrm{S}}$,

where $\alpha$ is the fraction of power from the microwave source which is lost in waveguides, ferrite attenuators, cavity walls, and diagnostic loads. In these experiments, we estimate $\alpha \approx 0.35$; many of these losses were introduced deliberately. The amount of "toroidal" power supplied may be compared to the toroidal plasma energy density to estimate the average energy confinement time $\left\langle\tau_{E}\right\rangle$ of the toroidal plasma; i.e.,

$\left\langle\tau_{E}>=\frac{\int\left(n_{e} T_{e}+n_{i} T_{i}\right) d V_{\text {toroidal }}}{P_{\text {toroidal }}}\right.$.

In EBT-I it was found that the various components absorb comparable amounts of power, and so in taking the difference in Eq. (15), relatively modest uncertainties in each component are additive and result in a rather wide uncertainty in $\mathrm{P}_{\text {toroidal }}$. The power to the annulus, $\mathrm{P}_{\mathrm{A}}=\mathrm{n}_{\mathrm{A}}<\mathrm{E}_{\mathrm{A}}>\mathrm{V}_{\mathrm{A}} / \tau$, is estimated by observing $\mathrm{W}_{\perp}=\int \mathrm{nEdV}$ diamagnetically and dividing by the decay time observed on turnoff of the power. This latter quantity cannot be determined if, on power turnoff, the stabilizing cold plasma density is reduced to where the annulus becomes unstable. Thus, decays are generally measured at the higher pressures where the cold plasma density can be regenerated during decay to maintain stability and with some residual power left on. The added energy loss due to 
regeneration may have some influence on the decay time, but more importantly, the range of conditions under which decays can be measured is necessarily limited. Nevertheless, the value obtained for $\tau_{A}$ is $0.1 \mathrm{sec}$; from this we estimate $\mathrm{P}_{\mathrm{A}}=40 / 0.1 \times 24=9.6 \mathrm{~kW}$, representing $\sim 26 \%$ of the applied power.

Considerably greater uncertainty exists in the estimate of $\mathrm{P}_{\mathrm{S}}$ because its determination must rely on more indirect evidence. To estimate $P_{s}=\int n_{s} E_{s} d V_{s} / \tau_{s}$ we are forced to determine each of the factors independently, with each contributing its own uncertainty. The surface plasma density is derived from the Langmuir probe ion saturation current $\bar{r}_{e}=3 \times 10^{11} \mathrm{~cm}^{-3}$. The electron temperature is $30 \mathrm{eV}$ from measurements of helium spectroscopic line ratios. Both the density and effective charge of impurities are relatively low, and the product compared to the electron density is also small; thus, the power lost to radiation and impurity ionization is $<1 \mathrm{~kW}$.

The ion energy is not directly measured, but is presumed to be in the range of $\sim 1-10 \mathrm{eV}$. The ionization and excitation energy for hydrogen contributes $\sim 30 \mathrm{eV}$ to the energy balance. Including charge exchange and impurities, we therefore estimate the total energy taken out of the plasma by each ion pair to be $\sim 100 \mathrm{eV}$. The volume of surface plasma, based on the optical spatial evidence i.s

$V=(2 \pi r \Delta r)(2 \pi R) \cong 5 \times 10^{5} \mathrm{~cm}^{3}$

Two estimates of surface plasma confinement time exist. From impurity charge state ratios we estimate $\tau_{\mathrm{s}} \cong 300 \mu \mathrm{sec}$. Density decay measurements using Langmuir probes following power turnoff indicate $\tau \backsim 100 \mu \mathrm{sec}$; thus $\mathrm{P}_{\mathrm{S}}=5-15 \mathrm{~kW}$.

It we take $\mathrm{P}_{\mathrm{S}}=10 \mathrm{~kW}, \mathrm{P}_{\mathrm{A}}=10 \mathrm{~kW}$, and $\alpha=0.35$, then $\mathrm{P}_{\text {toroidal }}=$ $16 \mathrm{~kW}$ and $\left\langle\tau_{E}\right\rangle=\int \mathrm{n}_{e}\left(\mathrm{~T}_{e}+\mathrm{T}_{i}\right) \mathrm{dV} / \mathrm{P}_{\text {toroidal }}=3 \mathrm{msec}$ [assuming $\mathrm{n}_{\mathrm{e}}(\mathrm{r})=$ const., $T_{e}(r)=$ const.]. With the range of uncertainty of $P_{s}$ our best estimate of $\left\langle\tau_{E}\right\rangle=2-8 \mathrm{msec}$. 


\subsection{PARAMETRIC STUDIES}

As discussed in the earlier sections, EBT-I was not designed with the intention of examining plasma transport properties because confinement times were expected to be controlled by atomic processes in the relatively low magnetic field and small plasma diameter. However, following the developments in the diagnostic program, it became apparent that plasma confinement was sufficiently good to be worthy of study.

The measurements of neutral density at the center of the toroidal plasma remain a difficult problem. The value obtained from iterative algebraic reconstruction of spatially resolved spectroscopic data is $\mathrm{n}_{\mathrm{o}} \sim 10^{10} \mathrm{~cm}^{-3}$, while the charge-exchange neutral spectrometer data imply a value somewhat lower. Neither measurement is without difficulty, but the apparent inconsistency is yet to be resolved by future experiments. The higher (spectroscopic) value of neutral density implies (with $\langle\sigma v\rangle_{\text {ion }}=3.0 \times 10^{-8}$ ) that the particle replacement time $\tau_{0}$ is $3.3 \mathrm{msec}\left(\tau_{0}=1 / \mathrm{n}_{0}\langle\sigma v\rangle_{i o n}\right)$. In any case, the particle replacement time is apparently sufficiently long that a range of plasma parameters may be fruitfully studied to permit comparison with the neoclassical transport model which was developed in parallel with the experimental observation. An example of the range of parameters available for theoretical comparison is indicated in Fig. $30(\mathrm{a}, \mathrm{b})$. In this figure we show the electron temperature and electron density observed as a function of neutral density (ambient pressure read on the ion gages) as determined from the soft $x$-ray bremsstrahlung measurements. The data are presented for several microwave power conditions, both with and without profile heating. The spread in experimental points indicates the variations in plasma conditions with nearly identical gage readings which result primarily from differences in vacuum wall conditions and the usual incremental drift of vacuum ionization gages. In spite of day-to-day variations, definitive trends are indicated as listed below.

(1) The electron temperature rises as the ambient neutral pressure is decreased. 

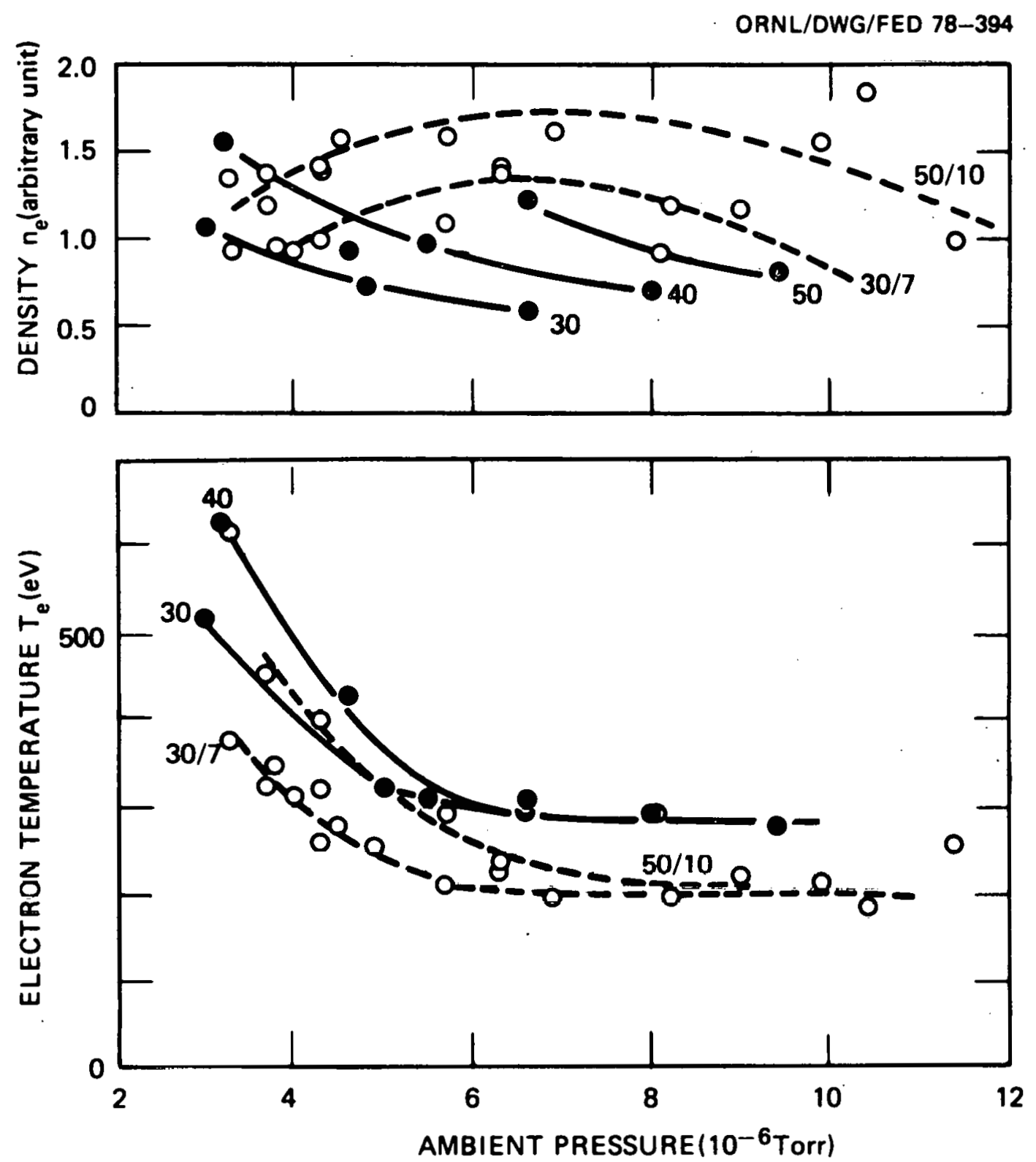

Fig. 30(a). Electron temperature, derived from bremsstrahlung measurements as a function of ambient neutral gas pressure with various microwave heating power combinactons at 18.0 and $10.6 \mathrm{GHz}$. (b) Lilectron density in toroidal plasma, derived from bremsstrahlung measurements as a function of ambient neutral gas measurements with various microwave heating power combinations at 18.0 and $10.6 \mathrm{GHz}$. 
(2) Higher temperatures are observed as the microwave power is increased, with $\mathrm{T}_{\mathrm{e}} \sim \sqrt{\mathrm{P}_{\mu}}$ (Fig. 31).

(3) Higher temperatures are observed without profile heating, but the observed densities are systematically lower under these conditions than when profile heating is used.

(4) The range of observations in T-mode is extended to higher neutral densities when profile heating is added to sustain the stabilizing annuli.

Figure 32 shows a plot for ion temperatures, which is similar to the plot in Fig. 30 for electron temperature, as a function of ambient pressure. Although fewer data points are available, the same trends as observed for the electron temperature are apparent. The data in Fig. 32 indicate the qualitative (but not quantitative) agreement observed for measurements taken with the nitrogen and cesium cells.

Quantitative results obtained using the charge-exchange neutral particle analyzer must be considered presently to be tentative for two reasons. The calibrations used in correcting the observed rates are presently under intensive study; for example, the secondary emission coefficients have just been revised and calibration system refinements incorporated at the lower energies. Secondly, the ion temperatures are just at the limit of measurability, generally from observations of charge-exchange flux for $E>T_{i}$. The most recent reevaluation of the data suggests nonuniform radial profiles of $\mathrm{n}_{0}$ and $\mathrm{T}_{i}$. However, as discussed in Sect. 4, the quantitative analysis of the observed fluxes leaves uncertainties which can only be removed by better spatially resolved measurements. In any case, as we discuss below, the primary contribution to the stored energy resides in the electron population, and the transport is theoretically dominated by this species as well, so the uncertainties associated with the ion measurements are not critical. With the expected temperature rise in future experiments, we anticipate that improved information on ion distributions will be obtained, both in configuration and velocity space.

In Fig. 33 we present the results of spatial mapping of the space potential measured with the heary ion beam probe. In this figure we 


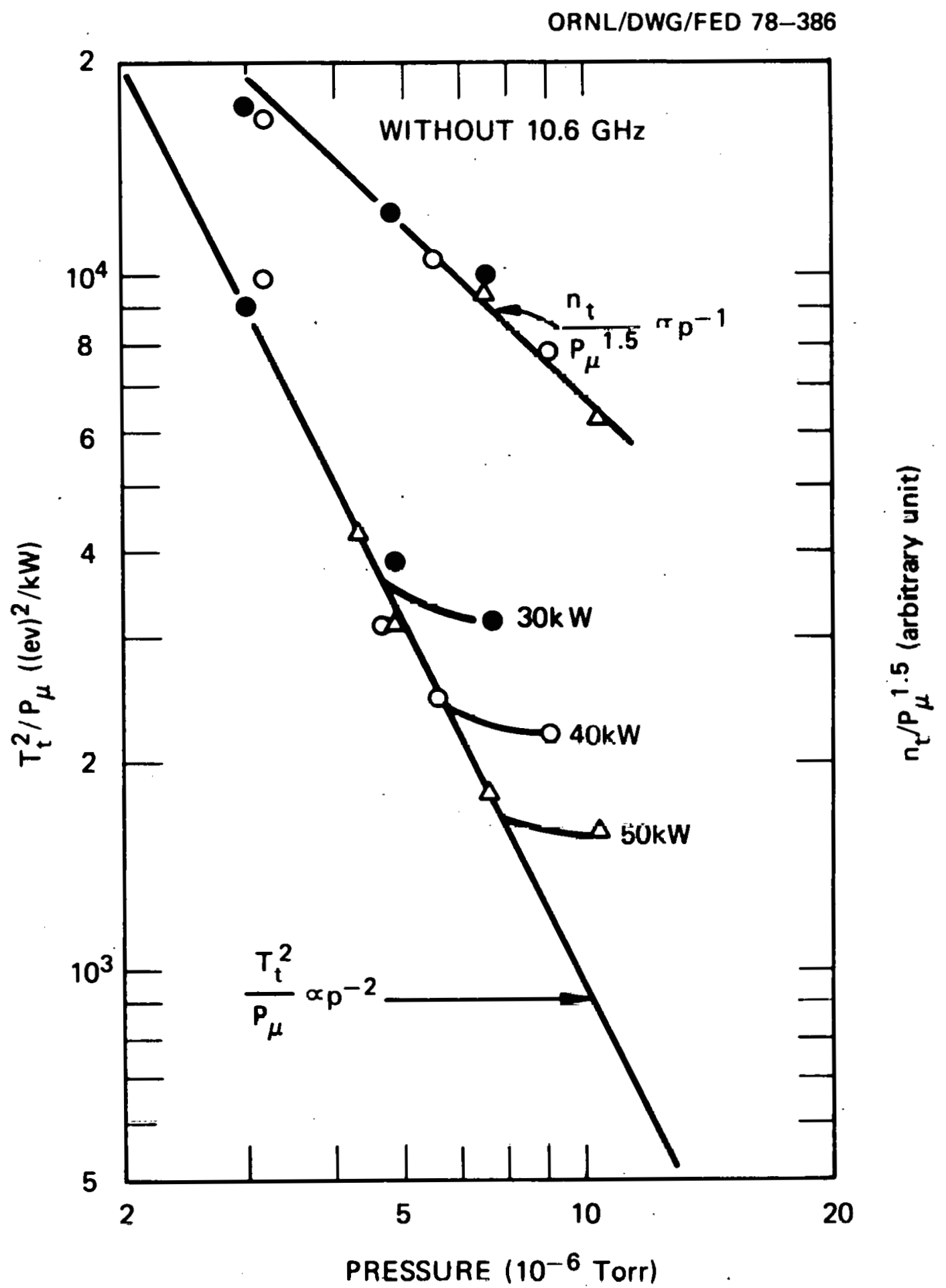

Fig. 31. Electron temperature divided by (microwave power) ${ }^{2}$ for data in Fig. 30 plotted as a function of ambient neutral pressure. The points shown are only for runs without profile heating. 
ORNL / DWG / FED 78-619R

$T_{i}$ vs $P_{0}$ Determined by
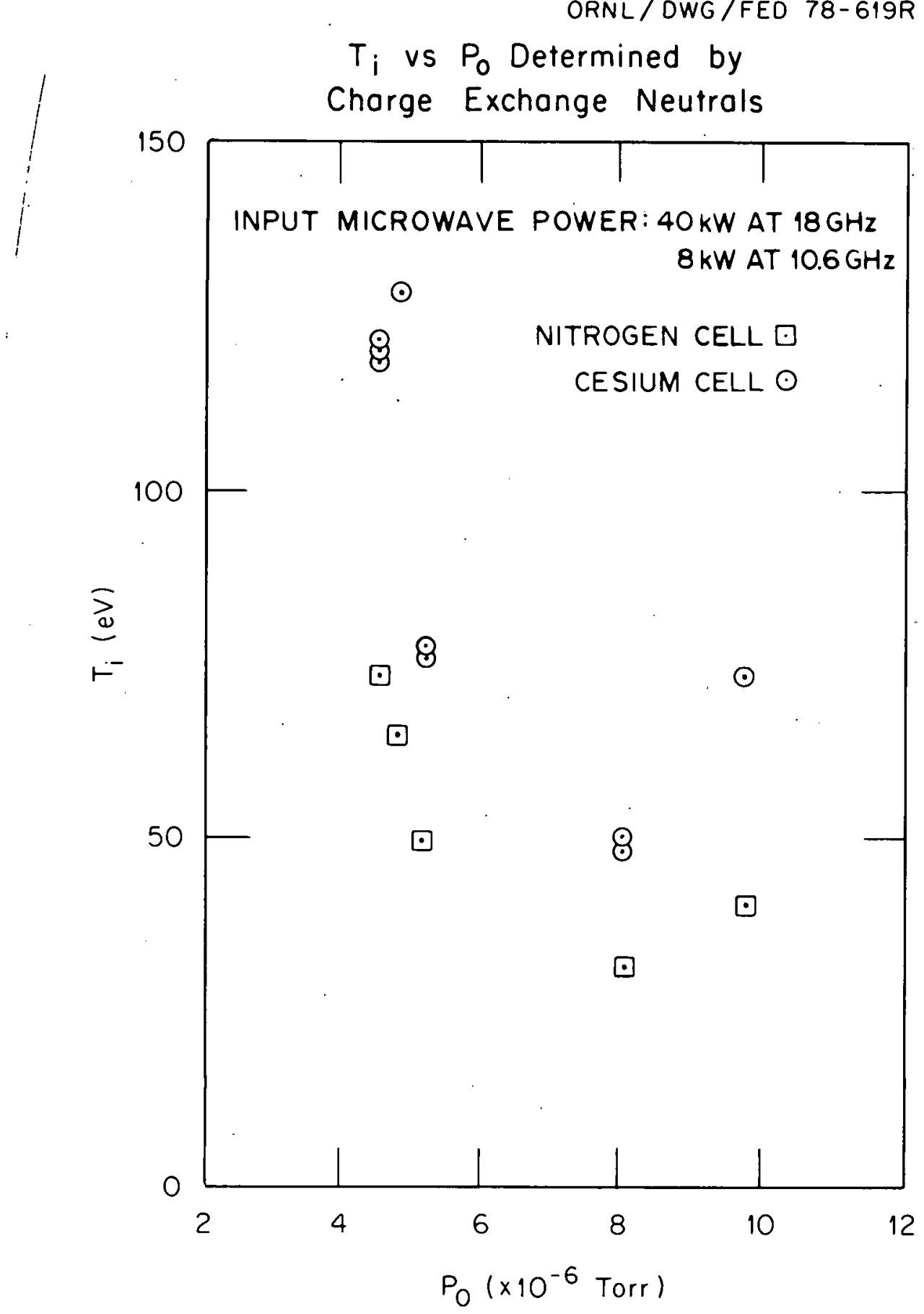

Fig. 32. Ion temperatures obtained from chargeexchange neutral measurements using the cesium cell reionizer and nitrogen stripping cell as a function of ambient neutral pressure with $40 \mathrm{~kW}$ of $18 \mathrm{GHz}$ and $8 \mathrm{~kW}$ of $10.6 \mathrm{GHz}$ microwave power. 
ORNL/DWG/FED-78-508

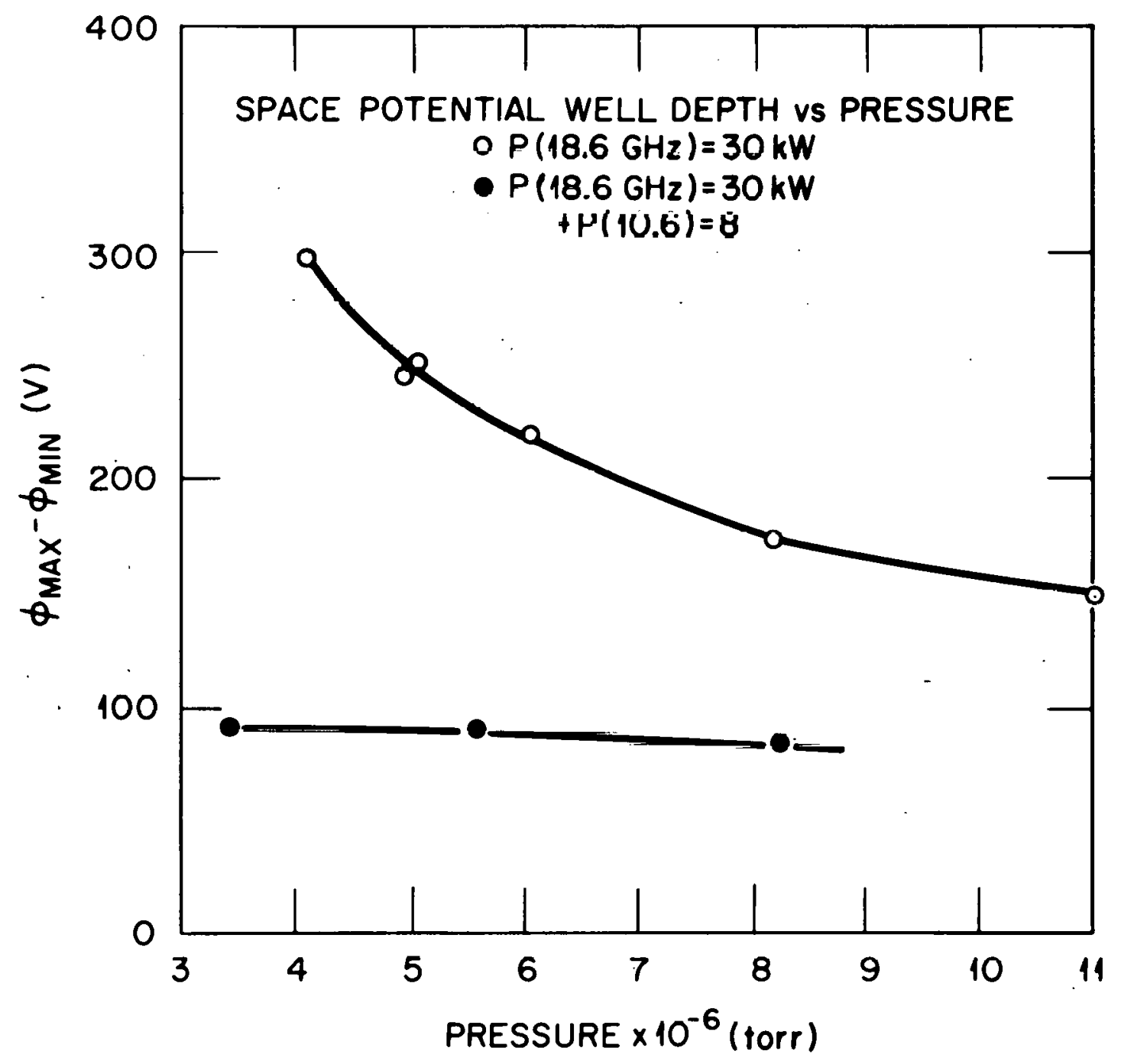

Fig. 33. Potential well depth (difference between maximum and minimum space potential) determined from spatial scans of heavy ion beam probe as a function of ambient neutral density for fixed heating power $[P(18 \mathrm{GHz})=30 \mathrm{~kW}, P(10.6 \mathrm{GHz})=8]$. 
plot the difference between the maximum and minimum observed potential $\left(\Delta \phi_{S}\right)$ as a function of ambient pressure and note the trend of increasing $\Delta \phi_{S}$ with decreasing pressure, although in these cases this trend is demonstrable only in the data without profile heating. Because $\Delta \phi_{S}$ is presumably connected in a simple way to $\mathrm{T}_{e}$, these points tend to confirm the higher temperatures observed when profile heating is not applied and the more systematic behavior of the data for these conditions. This apparent more systematic trend is based on only a limited data base and must be more carefully studied in future experiments.

In order to make a comparison with theoretical predictions, we have chosen to recategorize this data in terms of the theoretically relevant dimensionless parameter $\nu / \Omega$, the ratio of collision frequency to poloidal precession frequency. To illustrate the significance of this parameter, we present a brlef heuristic description of the model used to describe the confinement of the toroidal plasma in EBT. In this model it is assumed that the plasma confined on primarily closed drift surfaces (i.e., the toroidal plasma) diffuses radially outward to radi1 where drift surfaces are primarily open, i.e., where particles may be lost to walls along flux lines. The effective step size for this diffusive process is the displacement of the centers of nearly circular drift orbits. In this approximation,

$\mathrm{D}_{\perp}=(\overline{\Delta \mathrm{x}})^{2} v$

with

$(\overline{\Delta x})^{2}=\left(\frac{v_{T}}{\Omega}\right)^{2} \cdot\left(\frac{\Omega^{2}}{\nu^{2}+\Omega^{2}}\right)$,

where $v_{T}=(c \mathrm{~T} / \mathrm{e})\left(\nabla_{\mathrm{T}} \mathrm{B} / \mathrm{B}^{2}\right)$ is the drift velocity due to the toroidal field curvature, and $\Omega=v_{p} / r=(c T / e) \nabla_{m} B / r B^{2}+c E / r B$ is the poloidal drift frequency due to the mirror field gradient and electric field. The factor $\Omega^{2} /\left(\nu^{2}+\Omega^{2}\right)$ is included to take into account the more 
collisional regimes where particles are unable to complete their poloidal drift about the axis in the time $v^{-1}$.

This simple model results in the form of the particle diffusion coefficient used by Kovrizhnikh ${ }^{6}$ for describing nonaxisymmetric systems

$\mathrm{D}=\left(\frac{\mathrm{v}_{\mathrm{T}}}{\Omega}\right)^{2} \frac{\Omega^{2}}{\nu^{2}+\Omega^{2}} \nu$

Two limits of collisionality may be identified as

$\nu$ >. $\Omega: \quad D=\frac{\left(v_{T}\right)^{2}}{v}$. and $\quad v \ll s: \quad D=\left(\frac{v_{T}}{s i}\right)^{2} \nu$,

Extreme caution must be excrcised when using these essentially dimensional formulae for scaling parameters in bumpy tori. In carrying out detailed calculations of transport properties, energy balance equations as well as particle balance equations must be included. The quantitative factors multiplying the various gradients which drive diffusion in the heat and particle transport equations are found in kinematic calculations and can yield very different results when all physical properties are properly taken into account. For example, the electric field drift in the poloidal precession frequency is in opposite directions relative to the magnetic field precession for the two signs of charged particles. More significantly, the electric field affects particular particles in the distrihution in a drastic way - those for which the magnetic gradient and electric field drifts cancel. For sufficiently low collisionality, some particles may even be permitted to leave on a drift time. Other particles may not execute simple precession about the axis but can have qualitatively different step sizes (i.e., banana orbits). The theoretical problem of dealing quantitatively with all the different classes of particles as a function of position has occupied the theoretical effort for some time.

A summary of the kinematical calculations of the transport coefficients was recently completed by Spong, Harris, and Hedrick ${ }^{8}$. In this 
review although some of the effects of the electric field on the ion orbits were not included (e.g., banana orbits), most of the other principal mechanisms which affect electron transport were evaluated. In recent studies, it has become clearer that in the existing regimes of electron and ion temperature the electron transport qualitatively controls the ambipolar flux and dominates energy loss. Thus, a comparison of the energy confinement time with the electron heat transport coefficient would be expected to show the proper functional trends. Spong et al. have derived an "averaged" normalized heat coefficient and energy confinement time as a function of "averaged" $\nu / \Omega$. They give

$$
\frac{\nu}{\Omega}=2.7 \times 10^{-7} \frac{\mathrm{n}_{\mathrm{e}}}{\mathrm{T}_{\mathrm{e}}^{5 / 2}} \frac{1}{\left[1+0.7\left(\Delta \phi_{\mathrm{S}} / \mathrm{T}_{\mathrm{e}}\right)\right]}
$$

where the constant is evaluated for EBT-I conditions: $B_{00}=0.5 \mathrm{~T}$, plasma radius $a=10 \mathrm{~cm}$, and major toroidal radius $\mathrm{R}=150 \mathrm{~cm}$. In the model, density and temperature radial distributions were assumed to be Bessel functions, and the potential profile was taken to be parabolic. The energy confinement time was then calculated explicitly by spong et al. as a function of $\nu / \Omega$. Their normalized form of the energy confinement time, $\tau_{E}^{\prime}$, determined from the heat transport coefficients ${ }^{8}$ $\mathrm{K}_{\mathrm{n}}^{\prime}$ and $\mathrm{K}_{\mathrm{T}}^{\prime}$

$\frac{1}{\tau_{E}^{\prime}}=\left(\frac{1}{T_{E^{2} \tau_{n}}}\right)=\left[K_{n}^{\prime}\left(1+\frac{\Delta \phi_{O}}{\mathrm{~T}}\right)+K_{T}^{\prime}\right]$

is plotted vs $\nu / \Omega$ in Fig. 34; the points obtained from the data are also shown. In examining the data, it became apparent that insutficient experimental information was available to establish the power absorption fractions in the annulus and surface plasma for each plasma condition. Thus, the simple assumption was made that the relative power division. among the various components was unchanged from case to case. In this case, the relative value $\tau_{E}$ was calculated from the simple formula 


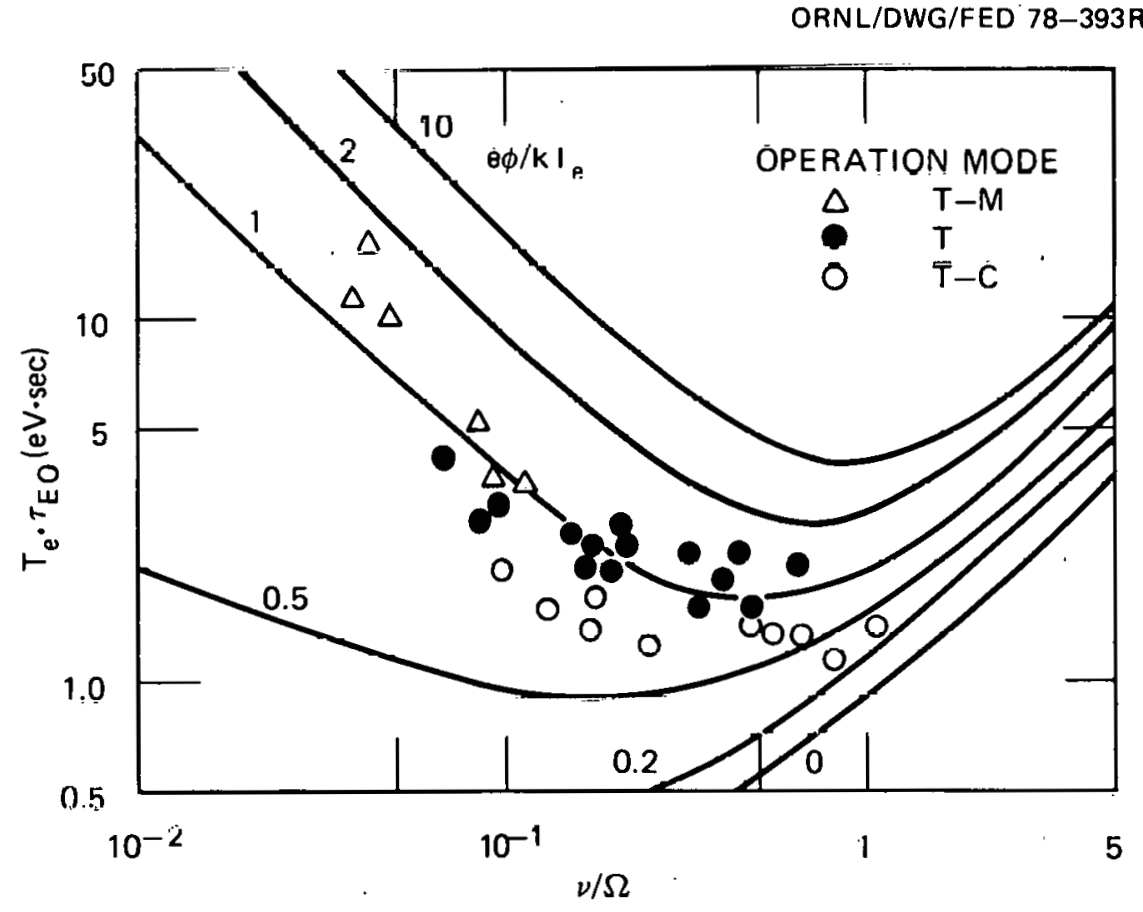

Fig. 34. Normalized relative average energy confinement time $\left(\tau_{E} T_{e}\right)$ as a function of collisionality parameter $\nu / \Omega$. Solid lines are energy confinement times alculated by Spong et al. for potentials indicated (negative electric fields). Points are $\left\langle\tau{ }_{E}\right\rangle=\bar{n} e^{T} e^{V / P_{\mu}}$
derived from data in Fig. 30 . 
$\tau_{E}=\frac{(3 / 2) n_{e} T_{e} V_{T}}{P_{\mu}}$.

The striking similarity in trend between the data points and theory cannot be ignored, particularly when it is recognized that for the points observed, $\Delta \phi / \mathrm{T}_{\mathrm{e}} \sim 0.5$ in good agreement with the shape of the curve $\Delta \phi_{\mathrm{s}} / \mathrm{T}_{\mathrm{e}}=1$.

This apparent agreement between experimentally line-averaged quantities $\left(\bar{n}_{e}, \bar{T}_{e}\right)$ and theoretically model-averaged diffusion coefficients is significant also because of the unforced comparison applied. The theory is based solely on classical collisional diffusive processes in our geometry (instabilities are not seen experimentally), and the points extend continuously over the parameter range $\nu / \Omega$ for which data could be accumulated. Some stable regimes at higher values of $\nu / \Omega$ have been observed experimentally, but the electron temperatures under these conditions are lower and therefore outside the range of measurability using bremsstrahlung as a diagnostic tool. In fact, because of the limitations imposed by microwave power cutoff at densities only slightly higher than those quoted, the entire range $\nu / \Omega \gg 1$ may only be accessible at a higher frequency.

This compilation of results constitutes the basis for our present understanding of the EBT plasma. The experiments have demonstrated the validity of the concept of macroscopically stable confinement of warm plasma in a bumpy torus stabilized by diamagnetic annuli. The plasma parameters are modest, but with the low magnetic field and available power these parameters are nevertheless significant. A theoretical model is presently in development to quantitatively describe these results and allow projections in more fusion-relevant regimes. It has been determined that such a model must include considerations of particle motions which in more symmetric geometries may, to first order, be ignored. However, even the simplest considerations lead to a formulation which describes many of the detailed features observed in the experiment, but presently with insufficient accuracy to permit confident extrapolations into reactor regimes. We anticipate from expected 
developments in the near future, both experimentally (EBT-S) and theoretically, that expanded understanding will permit a more realistic evaluation of the desirability of the EBT configuration as a fusion reactor. 


\section{REFERENCES}

1. R. A. Dand1, H. O. Eason, A. C. England, G. E. Guest, C. L. Hedrick, and J. C. Sprott, ORNL/TM-3694, Oak Ridge, Tennessee (November 1971).

2. M. Bacal, A. True, H. J. Doucet, H. Lamain, and M. Chrétien, Nuc1. Instrum. Methods 114, 407-409 (1974); M. Bacal and W. Reichet, Rev. Sci. Instrum. 45, 769-772 (1974).

3. F. C. Jobes and R. L. Hickok, Nuc1. Fusion 10, 195 (1970).

4. An early review of various ART as applied to medical tomography is presented in IEEE Trans. on Nucl. Science, Vol. NS-21 (June 1974); see also Physics Today 30 32-41 (1977).

5. T. Jernigan, J. Rudmin, and D. M. Meade, Phys. Rev. Lett. 26, 1298 (1971); M. Okabayashi and R. Freemen, Princeton Plasma Physics Laboratory Report MATT-870, Princeton, New Jersey (November 1977).

6. L. M. Kovrizhnikh, Zh. Eksp. Teor. Fiz. [Sov. Phys.-JETP] 29, 475 (1969).

7. D. M. Meade and R. J. Fonck, Phys. Fluids 16, 1654 (1973).

8. D. A. Spong, E. G. Harris, and C. L. Hedrick, ORNL/TM-6215, Oak Ridge, Tennessee (April 1978). 
THIS PAGE

WAS INTENTIONALLY

LEFT BLANK 


\author{
ORNL/TM-6457 \\ Dist. Category UC-20 f, g
}

INTERNAL DISTRIBUTION

$\begin{array}{ll}\text { 1. } & \text { F. W. Baity, Jr. } \\ \text { 2. } & \text { C. F. Barnett } \\ \text { 3. } & \text { D. B. Batchelor } \\ \text { 4. } & \text { L. A. Berry } \\ \text { 5. F. M. Bieniosek } \\ \text { 6. J. D. Callen } \\ \text { 7. } & \text { J. A. Cobble } \\ \text { 8-70. R. A. Dand } 1 \\ \text { 71. R. A. Dory } \\ \text { 72. H. O. Eason, Jr. } \\ \text { 73. J. C. Glowienka } \\ \text { 74. G. R. Haste } \\ \text { 75. C. L. Hedrick, Jr. } \\ \text { 76. M. E. Hesse } \\ \text { 77. S. Hiroe } \\ \text { 78. E. F. Jaeger } \\ \text { 79. G. G. Kelley } \\ \text { 80. S. P. Kuo } \\ \text { 81. N. H. Lazar } \\ \text { 82. J. N. Luton }\end{array}$

\author{
83. O. B. Morgan, Jr. \\ 84. D. B. Nelson \\ 85. H. Postma \\ 86. B. H. Quon \\ 87. M. W. Rosenthal \\ 88. D. A. Spong \\ 89. N. A. Uckan \\ 90. T. Uckan \\ 91. T. L. White \\ 92-93. Laboratory Records \\ Department \\ 94. Laboratory Records, ORNL-RC \\ 95. Y-12 Document Reference \\ Section \\ 96-97. Central Research Library \\ 98. Fusion Energy Division \\ Library \\ 99. Fusion Energy Division \\ Communications Center \\ 100. ORNL Patent Office
}

\title{
EXTERNAL DISTRIBUTION
}

101. D. J. Anthony, Energy Systems and Technology Division, General Electric Co., 1 River Rd., Bldg. 23, Rm. 290, Schenectady, NY 12345

102. W. B. Ard, McDonnell Douglas-East, P.0. Box 516, St. Louis, MO 63166

103. C. Baker, Fusion Engineering Department, General Atomic Co., P.0. Box 81608, San Diego, CA 92138

104. R. E. Balzhiser, Electric Power Research Institute, 3412 Hillview Ave., P.0. Box 10412, Palo Alto, CA 94304

105. W. B. Briggs, McDonnel1 Douglas-East, P.O. Box 516, St. Louis, MO 63166

106. S. Buchsbaum, Research Communications Principles Division, Bell Telephone Laboratories, Inc., Murray Hill, NJ 07974

107. M. Clark, Combustion Engineering, Windsor, CT 06095

108. J. F. Clarke, Office of Fusion Energy (ETM), Mail Stop G-234, Department of Energy, Washington, DC 20545

109. F. H. Coensgen, Lawrence Livermore Laboratory, P.0. Box 808, Livermore, CA 94550

11U. F'. E. Lottman, Uttice of Fusion Energy (ETM), Mail Stop G-234, Department of Energy, Washington, DC 20545

111. P. L. Colestock, Plasma Physics Laboratory, Princeton University, P.Uं. Box 451, Princeton, NJ 08540 
112. R. W. Conn, University of Wisconsin, Department of Nuclear Engineering, Madison, WI 53706

113. T. Consoli, Centre d'Etudes Nucleaires de Grenoble, B.P. 85 Centre de Tri, 38041 Grenoble Cedex, Grenoble, France

114. E. C. Creutz, Bishop Museum, P.0. Box 6037, Honolulu, HI 96818

115. F. L. Culler, Electric Power Research Institute, 3412 Hillview Ave., P.o. Box 10412, Palo Alto, CA 94304

116. J. M. Dawson, Department of Physics, University of California, Los Angeles, CA 90024

117. S. O. Dean, Office of Fusion Energy (ETM), Mail Stop G-234, Department of Energy, Washington, DC 20545

118. R. DeBellis, McDonnell Douglas-East, P.O. Box 516, St. Louis, MO 63166

119. H. W. Deckman, Advanced Energy Systems Laboratory, Government Research Laboratories, Exxon Research and Engineering Co., P.o. Box 8, Linden, NJ 07036

120. D. A. Dingee, Manager, Fusion Programs, Battelle-Northwest, Battelle Blvd., P.O. Box 999, Richland, WA 99352

121. H. Dreicer, Group CTR-1, Los Alamos Scientific Laboratory, P.O. Box 1663, Los Alamos, NM 87545

122. R. A. Ellis, Plasma Physics Laboratory, Princeton University, P.0. Box 451, Princeton, NJ 08540

123. W. R. E11is, Jr., Office of Fusion Energy (ETM), Mail Stop G-234, Department of Energy, Washington, DC 20545

124. H. H. Fleischmann, Department of Applied Physics, Clark Hall, Cornell University, Ithaca, NY 14853

125. H. K. Forsen, Exxon Nuclear Co., 777 106th Ave. NE, Bellevue, WA y8u09

126. J. Foster, TRW Defense and Space Systems, 1 Space Park, Bldg. R-1, Redondo Beach, CA 90278

127. 'I'. K. Fowler, Lawrence Livermore Laboratory, P.U. Box 808, Livermore, CA 94550

128. M. Fujiwara, Institute of Plasma Physics, Nagoya University, Nagoya 464, Japan

129. H. P. Furth, Plasma Physics Laboratory, Princeton University, P.O. Box 451, Princeton, NJ 08540

130. Fusion Power Program, Argonne National Laboratory, $9700 \mathrm{~S}$. Cass Ave., Argonne, IL 60439

131. M. B. Gottlieb, Plasma Physics Laboratory, Princeton University, P.0. Box 451, Princeton, NJ 08540

132. R. W. Gould, California Institute of Technology, Mail Stop 116-81, Pasadena, C.A. 91109

133. H. Grad, New York University, Courant Institute of Mathematical Sciences, Magneto-Fluid Dynamics Division, 251 Mercer St., New York, NY 10012

134. A. Grant, TRW Defense and Space Systems, 1 Space Park, Bldg. R-1, Redondo Beach, CA 90278

135. G. E. Guest, General Atomic Co., P.0. Box 81608, San Diego, CA 92138

136. E. G. Harris, Department of Physics, University of Tennessee, Knoxville, TN 37916

137. I. G. Hedrick, Mail Stop C-0405, Grumman Aerospace Corp., Bethpage, NY 11714 
138. G. K. Hess, Jr., Office of Fusion Energy (ETM), Mail Stop G-234, Department of Energy, Washington, DC 20545

139. R. L. Hickok, Rensselaer Polytechnic Institute, ESE Department, Troy, NY 12181

140. A. G. Hill, Massachusetts Institute of Technology, Cambridge, MA 02139

141. R. L. Hirsch, Exxon Corp., Science and Technology Department, 1251 Avenue of the Americas, New York, NY 10020

142. J. Hosea, Plasma Physics Laboratory, Princeton University, P.0. Box 451, Princeton, NJ 08540

143. S. Humphries, Laboratory of Plasma Physics, 308 Upson Hall, Cornell University, Ithaca, NY 14853

144. R. Huse, Chairman, EPRI Fusion Program Committe, Public Service and Gas Co., 80 Park Place, Newark, NJ 07101

145. H. Ikegami, Institute of Plasma Physics, Nagoya University, Nagoya 464, Japan

146. H. R. Jory, Varian Associates, 611 Hansen Way, Palo Alto, CA 94303

147. A. Kadish, Office of Fusion Energy (ETM), Mail Stop G-234, Department of Energy, Washington, DC 20545

148. J. Killeen, Lawrence Livermore Laboratory, P.0. Box 808, Livermore, CA 94550

149. E. E. Kintner, Director, Office of Fusion Energy (ETM), Mail Stop G-234, Department of Energy, Washington, DC 20545

150. N. A. Krall, Science Applications, Inc., 1200 Prospect St., Box 2351, La Jolla, CA 92037

151. E. Lee, Controlled Thermonuclear Research Division, Lawrence Livermore Laboratory, P.0. Box 808, Livermore, CA 94550

152. Librarian, Institut für Plasmaphysik, 8046 Garching bei Munchen, Federal Republic of Germany

153. Librarian, Culham Laboratory, UK Atomic Energy Authority, Abingdon, Oxfordshire, England

154. Library, Centre de Recherches en Physique des Plasmas, 21 Avenue des Bains, 1007 Lausanne, Switzerland

155. D. G. McAlees, Exxon Nuclear Co., Inc., Research \& Technology Center, Laser Enrichment Department, 2955 George Washington Way, Richland, WA 99352

156. T. Mene, McDonnell Douglas-East, P.0. Box 516, St. Louis, MO 63166

157. R. Moir, Lawrence Livermore Laboratory, P.0. Box 808, Livermore, CA 94550

158. K. G. Moses, TRW Defense and Space Systems, 1 Space Park, B1dg. R-1, Redondo Beach, CA 90278

159. J. H. Mullen, McDonnell Douglas-East, P.O. Box 516, St. Louis, MO 6376h

160. S. Naymark, Nuclear Services Corp., 1700 Dell Ave., Campbe1l, CA 95008

161. T. Ohkawa, General Atomic Co., P.0. Box 81608, San Diego, CA 92138

162. L. D. Pearlstein, Lawrence Livermore Laboratory, P.0. Box 808, Livermore, CA 94550

163. Plasma Physics Library, Plasma Physics Laboratory, Princeton University, P.O. Box 451, Princeton, NJ 08540

164. R. E. Price, Chief, Fusion Plasma Theory Branch, Office of Fusion Energy (ETM), Mail Stop G-234, Department of Energy, Washington, DC 20545 
165. D. J. Rose, Department of Nuclear Engincering, Massachusetts Institute of Technology, Cambridge, MA 02139

166. F. R. Scott, Electric Power Research Institute, 3412 Hillview Ave., P.O. Box 10412; Palo Alto, CA 94304

167. Z. M. Shapiro, Westinghouse Electric Co., P.0. Box 355, Pittsburgh, PA 15230

168. W. M. Stacey, Jr., School of Nuclear Engineering, Georgia Institute of Technology, Atlanta, GA 30332

169. A. Staprans, Varian Associates, 611 Hansen Way, Palo Alto, CA 94303

170. H. S. Staten, Office of Fusion Energy (ETM), Mail Stop G-234, Department of Energy, Washington, DC 20545

171. F. Staudhammer, TRW Defense and Space Systems, 1 Space Fark, Bldg. R-1, Redondo Beach $\mathrm{C} \Lambda 90278$

172. C. My. Silckley, Laser Fuslon, Department of Energy, Washlngton, DC 20545

173. R. S. Symons, Varian Associates, 611 Hansen Way, Palo Alto,

174. F. Thomas, Grumman Aerospace Corp., Department 930, PLT 5, Bethpage, NY 11714

175. A. W. Trivelpiece, Exxon Nuclear Co., Inc., Research \& Technology Center, 2955 George Washington Way, Kichland, WA 99352

176. W. C. Turner, Lawrence Livermore Laboratory, P.0. Box 808, Livermore, CA 94550

177. C. von Keszycki, Grumman Aerospace Corp., Department 930, PLT 5, Bethpage, NY 11714

178. Director, Research and Technical Support Division, Department of Energy, Oak Ridge Operations, P.0. Box E, Oak Ridge, TN 37830

179-433. Given distribution as shown in TID-4500, Magnetic Fusion Energy (Distribution Category UC-20 f, g: Experimental Plasma Physics and Theoretical Plasma Physics) 